Supporting Information for:

\title{
Copolymerization of Ethylene and Alkyl Vinyl Ethers by a (Phosphine-sulfonate)PdMe Catalyst
}

\author{
Shuji Luo, Javier Vela, Graham R. Lief and Richard F. Jordan* \\ Department of Chemistry, The University of Chicago \\ 5735 South Ellis Avenue, Chicago, Illinois, 60637 \\ E-mail: rfjordan@uchicago.edu
}

\section{Contents}

Page

I. Methods and Materials

S2

II. Synthesis of (PO)PdMe(py)

S5

III. Stoichiometric Reaction of (PO)PdMe with $\mathrm{CH}_{2}=\mathrm{CHOEt}$

S7

IV. Copolymerization of ethylene and $\mathrm{CH}_{2}=\mathrm{CHOR}$

S9 


\section{Methods and Materials.}

General Information. All manipulations were performed using dry box or Schlenk techniques under purified nitrogen, or on a high-vacuum line unless indicated otherwise. Nitrogen was purified by passage through columns containing activated molecular sieves and Q5 oxygen scavenger.

Dichloromethane was dried over $\mathrm{CaH}_{2}$, stored over $\mathrm{P}_{2} \mathrm{O}_{5}$, and freshly vacuum transferred prior to use. Tetrahydrofuran was distilled from sodium/benzophenone. Pentane, hexanes, toluene and benzene were either distilled from sodium/benzophenone or purified by passage through columns of activated alumina and BASF R3-11 oxygen removal catalyst. $\mathrm{CD}_{2} \mathrm{Cl}_{2}$, $\mathrm{CDCl}_{3}$ and $\mathrm{C}_{6} \mathrm{D}_{5} \mathrm{Cl}$ were degassed by three freeze-pump-thaw cycles and dried over $\mathrm{P}_{2} \mathrm{O}_{5}$. N,N,N',N'-tetramethyl-ethane-1,2-diamine (tmeda) was distilled from $\mathrm{CaH}_{2}$. Pyridine was degassed by three freeze-pump-thaw cycles and dried over molecular sieves.

The compounds (tmeda) $\mathrm{PdCl}_{2}$ and (tmeda)PdMe $\mathrm{Pe}_{2}$ were prepared by literature procedures. ${ }^{1}$ $\mathrm{PdCl}_{2}$ was purchased from Strem. [ $\left.\mathrm{Li}\left(\mathrm{OEt}_{2}\right)_{\mathrm{n}}\right]\left[\mathrm{B}\left(\mathrm{C}_{6} \mathrm{~F}_{5}\right)_{4}\right]$ was purchased from Boulder Scientific. The $\mathrm{Et}_{2} \mathrm{O}$ content in $\left[\mathrm{Li}\left(\mathrm{Et}_{2} \mathrm{O}\right)_{\mathrm{n}}\right]\left[\mathrm{B}\left(\mathrm{C}_{6} \mathrm{~F}_{5}\right)_{4}\right]$ was determined by ${ }^{1} \mathrm{H} \mathrm{NMR}$ with $\mathrm{C}_{6} \mathrm{Me}_{6}$ as internal standard $(\mathrm{n}=2.8) . \mathrm{CH}_{2}=\mathrm{CHO}^{\mathrm{t}} \mathrm{Bu}, \mathrm{CH}_{2}=\mathrm{CHOEt}$ and $\mathrm{CH}_{2}=\mathrm{CHO}^{\mathrm{n}} \mathrm{Bu}$ were obtained from Aldrich, degassed by three freeze-pump-thaw cycles, and dried over $\mathrm{CaH}_{2} \cdot \mathrm{CH}_{2}=\mathrm{CHOSiPh}_{3}{ }^{2}$ and $\mathrm{CH}_{2}=\mathrm{CHOPh}^{3}$ were prepared using the general procedure described by Ishii. ${ }^{4}$ All other chemicals were purchased from Aldrich and used without further purification. Elemental analyses were performed by Midwest Microlab, LLC.

Gel permeation chromatography (GPC) measurements were performed on a Polymer Laboratories PL-GPC 220 instrument using 1,2,4-trichlorobenzene solvent (stabilized with 125 ppm BHT) at $150{ }^{\circ} \mathrm{C}$. A set of three PLgel $10 \mu \mathrm{m}$ Mixed columns was used. Samples were prepared at $160{ }^{\circ} \mathrm{C}$. Molecular weights were determined by GPC using narrow polystyrene standards and are corrected for linear polyethylene by universal calibration using the MarkHouwink parameters: $\mathrm{K}=1.75 \times 10^{-2} \mathrm{~cm}^{3} / \mathrm{g}$ and $\alpha=0.67$ for polystyrene and $\mathrm{K}=5.90 \times 10^{-2}$ $\mathrm{cm}^{3} / \mathrm{g}$ and $\alpha=0.69$ for polyethylene. ${ }^{5}$ 
NMR spectra were recorded on Bruker DMX-500 or DRX-400 spectrometers in Teflonvalved tubes at $20{ }^{\circ} \mathrm{C}$ unless specified otherwise. ${ }^{1} \mathrm{H}$ and ${ }^{13} \mathrm{C}$ chemical shifts are reported relative to $\mathrm{SiMe}_{4}$ and were determined by reference to the residual solvent signals. Coupling constants are reported in $\mathrm{Hz} .{ }^{13} \mathrm{C}$ NMR resonances were assigned with the assistance of DEPT-135 experiments.

NMR assignments for the vinyl ether units in ethylene $/ \mathrm{CH}_{2}=\mathrm{CHOR}$ copolymers were made based on literature data for model compounds (Chart S-1) and confirmed by COSY, HMQC and HMBC NMR spectra. NMR data for the model compounds in Chart 1 were obtained from Spectral Database for Organic Compounds (SDBS, see http://www.aist.go.jp/RIODB/SDBS/cgibin/cre index.cgi) and the literature. ${ }^{6}$ 
Chart S-1. NMR data for model compounds $\left(\mathrm{CDCl}_{3}\right.$, room temperature)

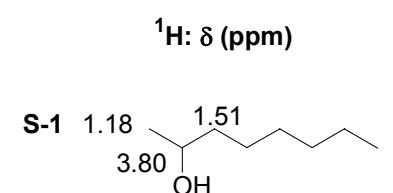

S-2

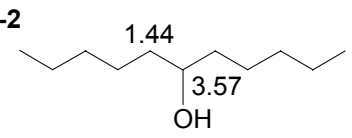

S-3

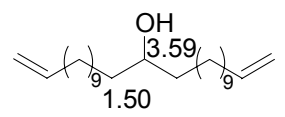

S-4

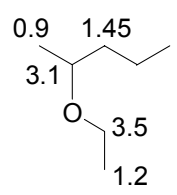

S-5

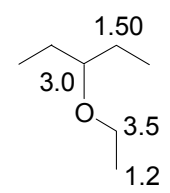

S-6

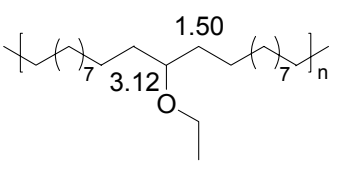

S-7

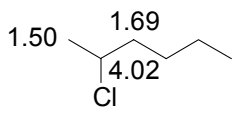

S-8

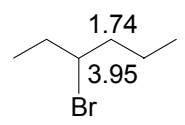

S-10

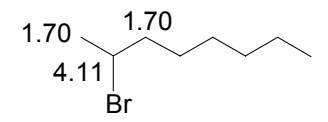

${ }^{13} \mathrm{C}: \delta(\mathrm{ppm})$
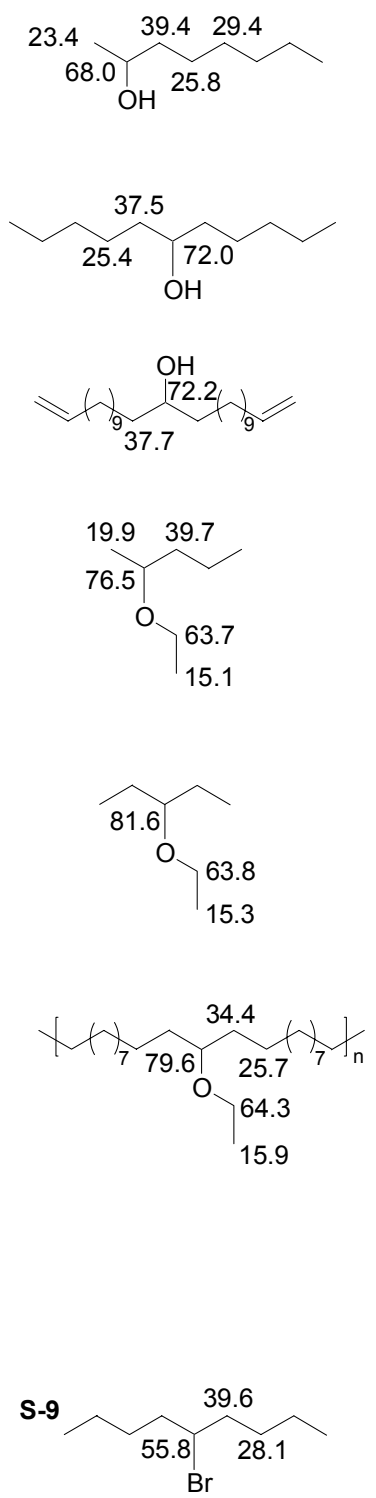

$\overbrace{\mathrm{Br}}^{26.5} \overbrace{27.8}^{41.3}$ 
II. Synthesis of (PO-OMe)PdMe(py). This complex was prepared using the route developed by Goodall et al. (eq S-1 $)^{7}$

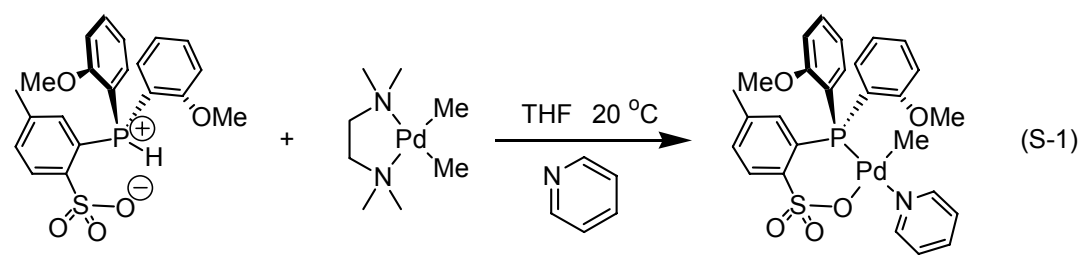

\section{Ortho-\{Bis(2-methoxyphenyl)phosphino\}toluenesulfonic acid ([PO-OMe]H) (GLA218).} A suspension of magnesium powder $(1.216 \mathrm{~g}, 50.00 \mathrm{mmol})$ in ether $(60 \mathrm{~mL})$ was cooled to $0{ }^{\circ} \mathrm{C}$, and 1-bromo-2-methoxybenzene $(5.50 \mathrm{~mL}, 40.0 \mathrm{mmol})$ was added slowly with vigorous stirring. The suspension was allowed to warm to room temperature, stirred for $16 \mathrm{~h}$, and filtered to yield a clear colorless solution of 2-methoxyphenyl magnesium bromide. The solution was cooled to 0 ${ }^{\circ} \mathrm{C}$ and a solution of $\mathrm{Cl}_{2} \mathrm{PNMe}_{2}(3.35 \mathrm{~g}, 19.3 \mathrm{mmol})$ in ether $(20 \mathrm{~mL})$ was added dropwise. The reaction mixture was allowed to warm to room temperature, stirred for $20 \mathrm{~h}$, and filtered yielding a yellow solution. The solvent was removed under vacuum yielding crude (2-OMe- $\mathrm{Ph})_{2} \mathrm{PNMe}_{2}$ as a viscous yellow oil $(5.58 \mathrm{~g})$. The oil was dissolved in ether $(100 \mathrm{~mL})$, and a solution of $\mathrm{HCl}$ in ether $(1 \mathrm{M}, 35.6 \mathrm{~mL}, 35.6 \mathrm{mmol})$ was added dropwise with vigorous stirring, causing the immediate formation of a white precipitate. The mixture was stirred for $1 \mathrm{~h}$ and filtered through a medium porosity frit to yield a colorless solution. The solvent was removed under vacuum to yield crude $(2-\mathrm{OMe}-\mathrm{Ph})_{2} \mathrm{PCl}$ as a white oil $(4.16 \mathrm{~g})$. In a separate flask, a THF solution $(60 \mathrm{~mL})$ of $p$-toluenesulfonic acid $(2.59 \mathrm{~g}, 15.0 \mathrm{mmol})$ was cooled to $0{ }^{\circ} \mathrm{C}$ and $n$-BuLi $(12.2 \mathrm{~mL}, 30.0$ mmol) was added dropwise. The resulting yellow mixture was allowed to warm to room temperature and was stirred for $14 \mathrm{~h}$. The mixture was cooled to $0{ }^{\circ} \mathrm{C}$, and a solution of $(\mathrm{OMe}-$ $\mathrm{Ph})_{2} \mathrm{PCl}(4.16 \mathrm{~g}, 15.0 \mathrm{mmol})$ in THF $(30 \mathrm{~mL})$ was added slowly. The mixture was allowed to warm to room temperature and was stirred for $24 \mathrm{~h}$. The solvent was removed under vacuum to yield an oily solid. Water $(100 \mathrm{~mL})$ was slowly added and the resulting cloudy mixture was acidified to $\mathrm{pH} 2$ using a $20 \% \mathrm{HCl} / \mathrm{H}_{2} \mathrm{O}$ solution. ${ }^{8}$ The mixture was extracted with $\mathrm{CH}_{2} \mathrm{Cl}_{2}(5 \mathrm{x}$ 
$20 \mathrm{~mL}$ ). The extracts were combined, dried over $\mathrm{MgSO}_{4}$, filtered, concentrated under vacuum, and placed in a freezer at $-20{ }^{\circ} \mathrm{C}$. Several fractions of colorless crystals were obtained by filtration. The crystals rapidly became opaque upon drying under vacuum. Yield $3.25 \mathrm{~g}$ (41\% based on $\left.\mathrm{Cl}_{2} \mathrm{PNMe}_{2}\right) .{ }^{1} \mathbf{H}$ NMR $\left(\mathrm{CD}_{2} \mathrm{Cl}_{2}, \mathrm{GL} 126.1 .1\right)$ : $\delta 9.40\left(\mathrm{~d}, J_{\mathrm{PH}}=600,1 \mathrm{H}, \mathrm{P}-H\right), 8.11(\mathrm{dd}$, $\left.J_{\mathrm{HH}}=8, J_{\mathrm{PH}}=5,1 \mathrm{H}, \mathrm{H}^{3}-\mathrm{ArSO}_{3}\right), 7.74\left(\mathrm{~m}, 2 \mathrm{H}, \mathrm{H}^{6}-\mathrm{ArOMe}\right), 7.57\left(\mathrm{~m}, 1 \mathrm{H}, \mathrm{H}^{4}-\mathrm{ArSO}_{3}\right), 7.14-7.07$ (m, 4H, $\mathrm{H}^{4}$-ArOMe, $\mathrm{H}^{5}$-ArOMe), 7.05 (m, 2H, $\mathrm{H}^{3}$-ArOMe), 6.96 (d, $\left.J_{\mathrm{PH}}=15,1 \mathrm{H}, \mathrm{H}^{6}-\mathrm{ArSO}_{3}\right)$, 3.81 (s, 3H, ArOMe), 2.30 (s, 3H, $\left.M e \mathrm{ArSO}_{3}\right) .{ }^{13} \mathbf{C}\left\{{ }^{1} \mathbf{H}\right\}$ NMR $\left(\mathrm{CD}_{2} \mathrm{Cl}_{2}, \mathrm{GL} 126.2 .1\right): \delta 162.0$ (s, $\left.\mathrm{C}^{2}-\mathrm{ArOMe}\right), 151.0\left(\mathrm{~d}, J_{\mathrm{PC}}=10, \mathrm{C}^{2}-\mathrm{ArSO}_{3}\right), 140.5\left(\mathrm{~d}, J_{\mathrm{PC}}=14, \mathrm{C}^{5}-\mathrm{ArSO}_{3}\right), 137.1\left(\mathrm{~s}, \mathrm{C}^{3}-\mathrm{ArOMe}\right)$, $135.8\left(\mathrm{~d}, J_{\mathrm{PC}}=2, \mathrm{C}^{4}-\mathrm{ArSO}_{3}\right), 135.2\left(\mathrm{~d}, J_{\mathrm{PC}}=8, \mathrm{C}^{5}-\mathrm{ArOMe}\right), 134.4\left(\mathrm{~d}, J_{\mathrm{PC}}=12, \mathrm{C}^{6}-\mathrm{ArSO}_{3}\right)$, $129.4\left(\mathrm{~d}, J_{\mathrm{PC}}=10, \mathrm{C}^{3}-\mathrm{ArSO}_{3}\right), 122.4\left(\mathrm{~d}, J_{\mathrm{PC}}=12, \mathrm{C}^{4}-\mathrm{ArOMe}\right), 113.1\left(\mathrm{~d}, J_{\mathrm{PC}}=99, \mathrm{C}^{1}-\mathrm{ArSO}_{3}\right)$, $112.5\left(\mathrm{~d}, J_{\mathrm{PC}}=6, \mathrm{C}^{6}-\mathrm{ArOMe}\right), 107.4\left(\mathrm{~d}, J_{\mathrm{PC}}=96, \mathrm{C}^{1}-\mathrm{ArOMe}\right), 57.0(\mathrm{~s}, \mathrm{ArOMe}), 21.5(\mathrm{~s}, M e-$ $\left.\mathrm{ArSO}_{3}\right) .{ }^{31} \mathbf{P}\left\{{ }^{1} \mathbf{H}\right\} \mathbf{N M R}\left(\mathrm{CD}_{2} \mathrm{Cl}_{2}, \mathrm{GL} 87.1 .1\right): \delta-11.0$ (br s).

Synthesis of (PO-OMe)PdMe(py). (NB3-P37) A flask was charged with (tmeda)PdMe $\mathrm{Pd}_{2}$ $(0.457 \mathrm{~g}, 1.81 \mathrm{mmol})$ and THF $(8 \mathrm{~mL})$ to yield a clear solution. (PO-OMe)H $(0.670 \mathrm{~g}, 1.61 \mathrm{mmol})$ was added and the white mixture was stirred vigorously for $40 \mathrm{~min}$. Pyridine $(0.75 \mathrm{~mL})$ was added by syringe and the mixture was vigorously stirred for $35 \mathrm{~min}$. The pale-yellow slurry was evaporated under vacuum to yield a white solid. The solid was washed with $\mathrm{Et}_{2} \mathrm{O}$ and dried under vacuum. $\mathrm{CH}_{2} \mathrm{Cl}_{2}$ was added and the mixture was stirred thoroughly for $1 \mathrm{~h}$ until a completely homogeneous solution was obtained. The solution was then evaporated under vacuum to yield a pale-yellow solid (0.77 g, 78\%). NMR data for (PO-OMe)PdMe(py): ${ }^{1} \mathbf{H}$ NMR $\left(\mathrm{CD}_{2} \mathrm{Cl}_{2}\right.$, NMR500-2, SL303.2): $\delta 8.76(\mathrm{~d}, J=5,2 \mathrm{H}$, o-Py), 7.94 (dd, $J=8,5 ; 1 \mathrm{H}), 7.87$ (t, $J=8,1 \mathrm{H}, \mathrm{p}-$ Py), 7.59 (br, 2H), 7.54 (t, $J=8,2 \mathrm{H}), 7.47$ (t, $J=7,2 \mathrm{H}, \mathrm{m}-\mathrm{Py}), 7.27$ (d, $J=8,1 \mathrm{H}), 7.09$ (d, $J=$ 12, 1H), $7.02(\mathrm{t}, J=8,2 \mathrm{H}), 6.98(\mathrm{dd}, J=8,5 ; 2 \mathrm{H}), 3.68(\mathrm{~s}, 6 \mathrm{H}, \mathrm{OMe}), 2.25(\mathrm{~s}, 3 \mathrm{H}$, tol-Me), 0.22 $\left(\mathrm{d}, J_{\mathrm{PH}}=3,3 \mathrm{H}, \mathrm{Pd} M e\right) .{ }^{13} \mathbf{C}\left\{{ }^{1} \mathbf{H}\right\}$ NMR $\left(\mathrm{CD}_{2} \mathrm{Cl}_{2}\right.$, OMEdisc. 2.1$): \delta 161.0\left(\mathrm{~s}, C^{2}-\mathrm{ArOMe}\right), 150.7$ (s, o-Py), $146.3\left(\mathrm{~d}, J_{\mathrm{PC}}=16.0, C^{2}-\mathrm{ArSO}_{3}\right), 138.9\left(\mathrm{~d}, J_{\mathrm{PC}}=8.0, C^{3}-\mathrm{ArSO}_{3}\right), 138.6\left(\mathrm{~s}, C^{6}-\mathrm{ArSO}_{3}\right)$, 137.8 (br, $C^{4}$-ArOMe), 135.4 (s, p-Py), 133.5 (s, m-Py), $131.1\left(\mathrm{~s}, C^{4}-\mathrm{ArSO}_{3}\right), 127.8\left(\mathrm{~d}, J_{\mathrm{PC}}=8.0\right.$, $\left.C^{5}-\mathrm{ArSO}_{3}\right), 127.5\left(\mathrm{~d}, J_{\mathrm{PC}}=50, C^{1}-\mathrm{ArSO}_{3}\right), 125.4\left(\mathrm{~s}, C^{5}-\mathrm{ArOMe}\right), 120.8\left(\mathrm{~d}, J_{\mathrm{PC}}=12, C^{6}-\mathrm{ArOMe}\right)$, 
$116.7\left(\mathrm{~d}, J_{\mathrm{PC}}=57, C^{1}-\mathrm{ArOMe}\right), 111.8\left(\mathrm{~s}, C^{3}-\mathrm{ArOMe}\right), 55.6(\mathrm{~s}, \mathrm{OMe}), 21.2\left(\mathrm{~s}, \mathrm{CH}_{3} \mathrm{ArSO}_{3}\right), 0.27$ $\left(\mathrm{d}, J_{\mathrm{PC}}=3.6, \mathrm{Pd} M e\right) .{ }^{31} \mathbf{P}\left\{{ }^{1} \mathrm{H}\right\}$ NMR $\left(\mathrm{CD}_{2} \mathrm{Cl}_{2}, J V 1100.22 .1\right): \delta 19.8$ (s). The analogous compounds (PO-OMe)PdR(py) $\left(\mathrm{R}=\mathrm{CH}_{2} \mathrm{SiMe}_{3}, \mathrm{CH}_{2}{ }^{\mathrm{t}} \mathrm{Bu}, \mathrm{CH}_{2} \mathrm{Ph}\right)$ were characterized by NMR and $\mathrm{EA}$, and for $\mathrm{R}=\mathrm{CH}_{2} \mathrm{SiMe}_{3}$, X-ray diffraction. ${ }^{9}$

Synthesis of (PO-OMe)PdMe. (eq S-2, NB3-P73) A flask was charged with (PO$\mathrm{OMe}) \mathrm{PdMe}\left(\right.$ py) $(62 \mathrm{mg}, 0.10 \mathrm{mmol}), \mathrm{B}\left(\mathrm{C}_{6} \mathrm{~F}_{5}\right)_{3}(60 \mathrm{mg}, 0.12 \mathrm{mmol})$ and $\mathrm{CH}_{2} \mathrm{Cl}_{2}(2 \mathrm{~mL})$. The mixture was stirred vigorously for $30 \mathrm{~min}$. A yellow solution formed. The volatiles were removed under vacuum to yield a yellow solid. The solid was washed thoroughly with toluene and dried under vacuum to yield an off-white solid (20 mg, 37\%). ${ }^{1} \mathbf{H}$ NMR $\left(\mathrm{CD}_{2} \mathrm{Cl}_{2}\right.$, NMR5001, SL349.3): $\delta 7.95$ (dd, $J=8,5 ; 1 \mathrm{H}), 7.54(\mathrm{t}, J=8,2 \mathrm{H}), 7.48(\mathrm{br}, 2 \mathrm{H}), 7.32(\mathrm{~d}, J=8,1 \mathrm{H}), 7.06$ (d, $J=12,1 \mathrm{H}), 6.99(\mathrm{~m}, 4 \mathrm{H}), 3.68(\mathrm{~s}, 6 \mathrm{H}, \mathrm{OMe}), 2.23$ (s, 3H, tol-Me), 0.44 (s, 3H, PdMe). The analogous compound $\left\{(\mathrm{PO}-\mathrm{OMe}) \mathrm{PdCH}_{2} \mathrm{SiMe}_{3}\right\}_{2}$ was characterized by NMR, EA and X-ray crystallography. ${ }^{9}$

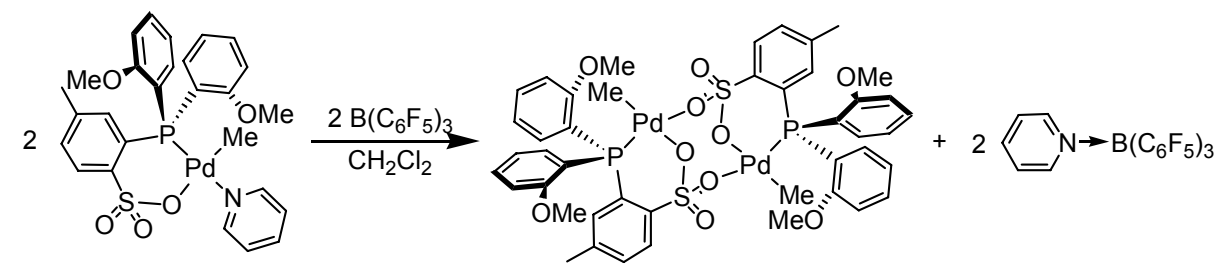

\section{Stoichiometric Reaction of (PO)PdMe with $\mathrm{CH}_{2}=\mathrm{CHOEt}$}

Reaction of (PO-OMe)PdMe with $\mathbf{C H}_{2}=$ CHOEt. (NB3-P74) An NMR tube was charged with (PO-OMe)PdMe (3.0 mg, $0.0025 \mathrm{mmol}) . \mathrm{CD}_{2} \mathrm{Cl}_{2}(0.4 \mathrm{~mL})$ was added by vacuum transfer at $-196{ }^{\circ} \mathrm{C}$. The tube was warmed to room temperature and shaken to form a pale yellow solution. $\mathrm{CH}_{2}=\mathrm{CHOEt}$ (16 equiv) was added by vacuum transfer at $-196{ }^{\circ} \mathrm{C}$. The tube was warmed to room temperature and shaken vigorously and monitored periodically by NMR at $24{ }^{\circ} \mathrm{C}$. After 8 $\min (\mathrm{PO}-\mathrm{OMe}) \mathrm{PdMe}\left(\mathrm{CH}_{2}=\mathrm{CHOEt}\right)(90 \%)$ and $(\mathrm{PO}-\mathrm{OMe}) \mathrm{PdCH}_{2} \mathrm{CH}(\mathrm{OEt}) \mathrm{Me}(10 \%)$ had formed. The $\mathrm{CH}_{2}=\mathrm{CHOEt}$ resonances were broad at $20^{\circ} \mathrm{C}$, showing that the exchange between free and coordinated $\mathrm{CH}_{2}=\mathrm{CHOEt}$ is fast on the NMR time scale at $20{ }^{\circ} \mathrm{C} .{ }^{10}$ After $2 \mathrm{~h}$, (PO$\mathrm{OMe}) \mathrm{PdMe}\left(\mathrm{CH}_{2}=\mathrm{CHOEt}\right)(15 \%)$ and $(\mathrm{PO}-\mathrm{OMe}) \mathrm{PdCH}_{2} \mathrm{CH}(\mathrm{OEt}) \mathrm{Me}(50 \%)$ were present. After 4 
h, $(\mathrm{PO}-\mathrm{OMe}) \mathrm{PdCH}_{2} \mathrm{CH}(\mathrm{OEt}) \mathrm{Me}(52 \%)$ and unidentified species $(48 \%)$ were present. The firstorder rate constant for the consumption of (PO-OMe) $\mathrm{PdMe}\left(\mathrm{CH}_{2}=\mathrm{CHOEt}\right)$ measured by the disappearance of the $\mathrm{Pd} M e{ }^{1} \mathrm{H}$ NMR resonance is $k_{\mathrm{obs}}=3.01(4) \times 10^{-4} \mathrm{~s}^{-1}$ at $24{ }^{\circ} \mathrm{C}$ (Figure S-1). After $1 \mathrm{~d}$, (PO-OMe) $\mathrm{PdCH}_{2} \mathrm{CH}(\mathrm{OEt}) \mathrm{Me}$ was consumed, and propylene and ethanol had formed. A multiplet at $\delta 5.60$ was present in the ${ }^{1} \mathrm{H}$ NMR, which is probably the $\mathrm{H}_{\text {int }}$ resonance of (PO$\mathrm{OMe}) \mathrm{Pd}\left(\eta^{3}-\mathrm{C}_{3} \mathrm{H}_{5}\right)$.

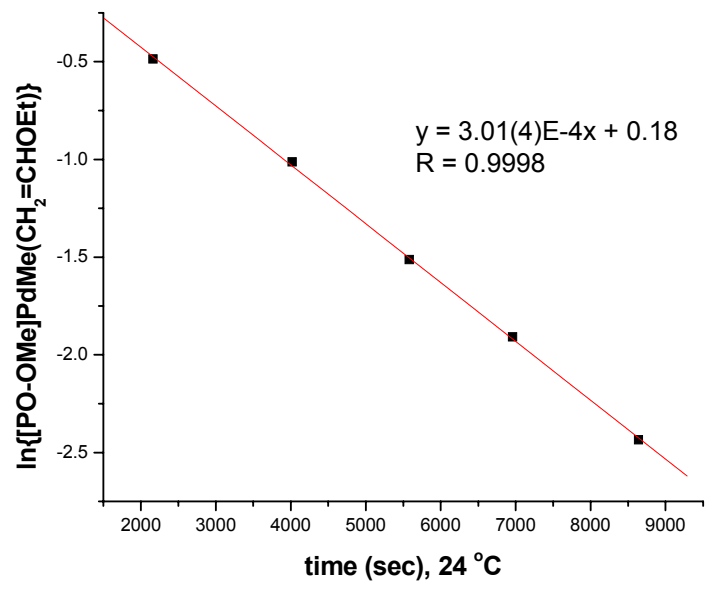

Figure S-1. First-order consumption of (PO-OMe) $\mathrm{PdMe}\left(\mathrm{CH}_{2}=\mathrm{CHOEt}\right)\left(24{ }^{\circ} \mathrm{C}\right)$

Key NMR data for (PO-OMe)PdMe(CH $\left.\mathrm{CH}_{2}=\mathbf{C H O E t}\right):{ }^{1} \mathbf{H}$ NMR $\left(\mathrm{CD}_{2} \mathrm{Cl}_{2}\right.$, NMR500-1, SL351-1): $\delta 7.94$ (dd, $J=8,5 ; 1 \mathrm{H}), 7.54$ (t, $J=8,2 \mathrm{H}), 7.35$ (br, 2H), 7.30 (d, $J=8,1 \mathrm{H}), 7.27$ (dd, $J=13,5 ; 1 \mathrm{H}, \mathrm{H}_{\text {int }}$ of $\left.\mathrm{CH}_{2}=\mathrm{CHOEt}\right), 7.01$ (t, $\left.J=9,3 \mathrm{H}\right), 6.98(\mathrm{dd}, J=8,5 ; 2 \mathrm{H}), 4.37$ (d, $J=6$, $1 \mathrm{H}, \mathrm{H}_{\text {cis }}$ of $\mathrm{CH}_{2}=\mathrm{CHOEt}$ ), 4.14 (q, $J=7,2 \mathrm{H}, \mathrm{OCH}_{2} \mathrm{CH}_{3}$ of $\left.\mathrm{CH}_{2}=\mathrm{CHOEt}\right), 4.12(\mathrm{~d}, J=14,1 \mathrm{H}$, $\mathrm{H}_{\text {trans }}$ of $\left.\mathrm{CH}_{2}=\mathrm{CHOEt}\right), 3.66(\mathrm{~s}, 6 \mathrm{H}, \mathrm{PO}-\mathrm{OMe}), 2.24\left(\mathrm{~s}, 3 \mathrm{H}\right.$, tol-Me), $0.17\left(\mathrm{~d}, J_{\mathrm{PH}}=3,3 \mathrm{H}\right.$, $\operatorname{Pd} \mathrm{Me}) .{ }^{11}$

Key NMR data for (PO-OMe)PdCH ${ }_{2} \mathbf{C H}(\mathrm{OEt}) \mathrm{Me}:{ }^{1} \mathbf{H}$ NMR $\left(\mathrm{CD}_{2} \mathrm{Cl}_{2}, \mathrm{NMR} 500-1\right.$, SL351-12): $\delta 4.73$ (sextet, $J=6,1 \mathrm{H}, \mathrm{PdCH}_{2} \mathrm{CH}(\mathrm{OEt}) \mathrm{Me}$ ), 3.71 (s, 6H, PO-OMe), 2.24 (s, 3H, tol-Me), 1.37 (t, $\left.J=7, \mathrm{OCH}_{2} \mathrm{CH}_{3}\right), 1.13$ (d, $\left.J=6,3 \mathrm{H}, \mathrm{PdCH}_{2} \mathrm{CH}(\mathrm{OEt}) M e\right), 0.89$ (dt, $J=8,3 ; 1 \mathrm{H}$, $\left.\mathrm{PdCH} \mathrm{H}^{\prime} \mathrm{CH}(\mathrm{OEt}) \mathrm{Me}\right), 0.37$ (t, $\left.J=7,1 \mathrm{H}, \mathrm{PdCH} H^{\prime} \mathrm{CH}(\mathrm{OEt}) \mathrm{Me}\right)$. These assignments were identified by comparison of NMR data for the analogous compound $[(\alpha-$ 
diimine $\left.) \mathrm{Pd}\left\{\mathrm{CH}_{2} \mathrm{CH}(\mathrm{OEt}) \mathrm{Me}\right\}\right]\left[\mathrm{B}\left(\mathrm{C}_{6} \mathrm{~F}_{5}\right)_{4}\right]$ and were confirmed by homonuclear decoupling experiments. $^{12}$

\section{Copolymerization of Ethylene and $\mathrm{CH}_{2}=\mathrm{CHOR}$.}

Comparative Ethylene Homopolymerization. (NB3-P47) A Fisher-Porter bottle equipped with a stir bar was charged with (PO-OMe)PdMe(py) $(3.1 \mathrm{mg}, 0.0050 \mathrm{mmol})$ and toluene $(25$ $\mathrm{mL})$. The bottle was pressurized with ethylene (20 psi, on demand), heated to $80{ }^{\circ} \mathrm{C}$ and stirred for $19 \mathrm{~h}$. The ethylene flow was stopped and the bottle was vented and cooled to room temperature. Methanol $(30 \mathrm{~mL})$ was added to complete the precipitation of the polymer. The polyethylene was washed with $\mathrm{CH}_{2} \mathrm{Cl}_{2}$ and acetone and dried under vacuum overnight to yield a white solid (1.43 g). GPC: $\mathrm{M}_{\mathrm{w}}=12,800, \mathrm{M}_{\mathrm{n}}=4,040$.

Copolymerization of Ethylene with $\mathrm{CH}_{2}=$ CHOEt. (NB3-P54) The procedure used above in the comparative ethylene homopolymerization was followed except that $\mathrm{CH}_{2}=\mathrm{CHOEt}$ was added. A Fisher-Porter bottle equipped with a stir bar was charged with (PO-OMe)PdMe(py) (3.1 $\mathrm{mg}, 0.0050 \mathrm{mmol}), \mathrm{CH}_{2}=\mathrm{CHOEt}(1.45 \mathrm{~g}, 19.0 \mathrm{mmol})$ and toluene (total solution volumn $25 \mathrm{~mL}$ ). The bottle was pressurized with ethylene ( $20 \mathrm{psi}$, on demand), heated to $80{ }^{\circ} \mathrm{C}$ and stirred for 19 h. The ethylene flow was stopped and the bottle was vented and cooled to room temperature. The mixture was evaporated under vacuum, washed with acetone and $\mathrm{CHCl}_{3}$ and dried under vacuum overnight to yield a white solid $(62 \mathrm{mg}) .{ }^{13}$ NMR spectra showed that this solid is an ethylene $/ \mathrm{CH}_{2}=\mathrm{CHOEt}$ copolymer with an ethylene $/ \mathrm{CH}_{2}=\mathrm{CHOEt}$ molar ratio $=95 / 5$. GPC: $\mathrm{M}_{\mathrm{w}}=$ $1680, M_{n}=934$. Other ethylene/vinyl ether copolymerizations were carried out using this general procedure, with some variation in conditions. Representative results are listed in Table S-1.

Effect of $\mathrm{H}_{2} \mathrm{O}$ on Copolymerization of Ethylene and $\mathrm{CH}_{2}=\mathrm{CHOEt}$. Two copolymerization reactions (entry 6, 7, Table S-1) were carried out under the same conditions except that in one case (entry 7), $\mathrm{H}_{2} \mathrm{O}(2 \mathrm{~mL})$ was added. The yield and molecular weight of resulting copolymer are slightly lower than those in entry 6. No significant difference was found for branching or vinyl ether incorporation level. 
Table S-1. Copolymerization of ethylene and $\mathrm{CH}_{2}=\mathrm{CHOR}\left(\mathbf{2 a - c}, \mathrm{R}={ }^{\mathrm{t}} \mathrm{Bu}(\mathbf{a})\right.$, Et (b), Bu (c) by (PO-OMe)PdMe(py)

\begin{tabular}{|c|c|c|c|c|c|c|c|c|c|}
\hline Entry & 1 & 2 & 3 & 4 & 5 & 6 & $7^{\mathrm{a}}$ & 8 & $9^{b}$ \\
\hline Exp \# & 312 & 325 & X19 & 319 & $\mathrm{X} 21$ & 353 & $353(2)$ & $310(2)$ & 308 \\
\hline$[\mathrm{Pd}](\mu \mathrm{mol})$ & 5 & 5 & 10 & 5 & 10 & 10 & 10 & 5 & 5 \\
\hline $\mathrm{T}\left({ }^{\circ} \mathrm{C}\right)$ & 80 & 80 & 80 & 80 & 80 & 100 & 100 & 80 & 80 \\
\hline$P_{\mathrm{CH} 2 \mathrm{CH} 2}(\mathrm{psi})$ & 20 & 20 & 74 & 80 & 294 & 300 & 300 & 20 & 20 \\
\hline $\mathrm{CH}_{2}=\mathrm{CHOR}$ & - & $2 \mathrm{~b}$ & - & $2 \mathbf{b b}$ & - & $2 \mathbf{b}$ & $2 \mathbf{b}$ & $2 c$ & $2 a$ \\
\hline $2(\mathrm{mmol})$ & 0 & 19 & 0 & 84 & 0 & 258 & 258 & 60 & 35 \\
\hline Volume (mL) & 25 & 25 & 50 & 25 & 50 & 50 & 50 & 25 & 25 \\
\hline Time (h) & 19 & 19 & 1 & 21 & 1 & 21 & 21 & 19 & 19 \\
\hline Yield $(\mathrm{g})$ & 1.4 & 0.06 & 1.5 & 0.28 & 3.6 & 2.1 & 1.4 & 0.10 & 0.09 \\
\hline $\mathrm{M}_{\mathrm{w}}\left(10^{3}\right) \mathrm{GPC}$ & 12.8 & 1.7 & 33.7 & 1.8 & 37.0 & 9.6 & 7.6 & & \\
\hline $\mathrm{M}_{\mathrm{n}}\left(10^{3}\right) \mathrm{GPC}$ & 4.0 & 0.9 & 13.5 & 0.9 & 17.6 & 4.8 & 3.6 & & \\
\hline $\mathrm{M}_{\mathrm{n}}\left(10^{3}\right) \mathrm{NMR}^{c}$ & 6.8 & 2.2 & 16.1 & 2.9 & 17.1 & 7.7 & 3.6 & 3.1 & 1.9 \\
\hline $\mathrm{Me} \mathrm{br/1000C}$ & 9.9 & 3.4 & 4.1 & 4.0 & 2.0 & 1.4 & 1.4 & 3.2 & 9.2 \\
\hline Incorp of $\mathbf{2}(\mathrm{mol} \%)$ & - & 4.9 & - & 4.2 & - & 2.0 & 2.1 & 6.9 & 1.2 \\
\hline $\mathbf{I}(\mathrm{mol} \%)^{d}$ & - & 3.5 & - & 2.7 & - & 1.4 & 1.4 & 4.8 & 0.2 \\
\hline II $(\mathrm{mol} \%)^{e}$ & - & 1.4 & - & 1.5 & - & 0.6 & 0.7 & 2.1 & 1.0 \\
\hline
\end{tabular}

${ }^{a} 2 \mathrm{~mL} \mathrm{H}_{2} \mathrm{O}$ was added to the solution before the reaction started.

$b$ The $\mathrm{CHCl}_{3}$ /acetone wash contains ethylene oligomers (number average degree of oligomerization ca. 10) with $\mathrm{CH}_{3} \mathrm{CH}\left(\mathrm{O}^{t} \mathrm{Bu}\right)$ - as a major head group.

${ }^{c}$ As calculated assuming that each chain contains one unsaturated end group. For the ethylene/vinyl ether copolymers, some chains may not contain unsaturated end groups, in which case the calculated $M_{n}$ will be higher than the true value.

${ }^{d} \mathbf{I}:-\mathrm{CH}_{2} \mathrm{CH}(\mathrm{OR}) \mathrm{CH}_{2-}$.

${ }^{e}$ II: $\mathrm{CH}_{3} \mathrm{CH}(\mathrm{OR}) \mathrm{CH}_{2}-$.

NMR Characterization of Ethylene $/ \mathbf{C H}_{2}=\mathbf{C H O}^{t} \mathbf{B u}$ Copolymer. The poly(ethylene-co$\mathrm{CH}_{2}=\mathrm{CHO}^{t} \mathrm{Bu}$ ) produced above is very similar to the polyethylene produced in the comparative ethylene homopolymerization described above. NMR studies show that the copolymer contains ca. 10 branches per $1000 \mathrm{C}$, most of which are methyl branches. Trace amounts of ethyl and propyl branches were also detected by ${ }^{13} \mathrm{C}$ NMR. The unsaturated units are vinyl groups ($\left.\mathrm{CH}_{2} \mathrm{CH}=\mathrm{CH}_{2}\right)$ and 2-olefins $\left(-\mathrm{CH}_{2} \mathrm{CH}=\mathrm{CHMe}\right)$. Additionally, the copolymer contains two major 
comonomer units, $-\mathrm{CH}_{2} \mathrm{CH}\left(\mathrm{O}^{t} \mathrm{Bu}\right) \mathrm{CH}_{2^{-}}$(I) and $\mathrm{CH}_{3} \mathrm{CH}\left(\mathrm{O}^{t} \mathrm{Bu}\right) \mathrm{CH}_{2^{-}}$(II), which have been identified by 1- and 2-D NMR and comparison with data for model compounds. A detailed discussion of how these assignments were made is provided in the next section. Representative 1- and 2-D NMR spectra of the copolymer are shown in Figures S-2a-h. The assignments for major comonomer units are listed below. Data for model compounds are listed in Chart S-1 (compounds S-1, S-2, S-3). All other resonances in the spectra of the copolymer are present in the spectra of homo-polyethylene. Resonances for the $-\left[\mathrm{CH}_{2} \mathrm{CH}\left(\mathrm{O}^{t} \mathrm{Bu}\right)\right]_{\mathrm{n}}$ - homopolymer (see Figures S-3a-f) were not observed in the spectra of poly(ethylene- $\left.c o-\mathrm{CH}_{2}=\mathrm{CHO}{ }^{\mathrm{t}} \mathrm{Bu}\right)$; in particular, compare Figure S-2b vs. Figure S-3b; Figure S-2f vs. Figure S-3d; Figure S-2g vs. Figure S-3e; and Figure S-2h vs. Figure S-3f.

NMR Assignments of Key Comonomer Units in Ethylene $/ \mathrm{CH}_{2}=\mathrm{CHO}^{t} \mathrm{Bu}$ Copolymer. Resonances for $\boldsymbol{- C H}\left(\mathrm{O}^{t} \boldsymbol{B} \boldsymbol{u}\right)$ - comonomer units were found in the ${ }^{1} \mathrm{H}$ NMR spectra (Figures S$2 \mathrm{~b}, \mathrm{c}$ ) and the ${ }^{13} \mathrm{C}$ NMR spectra (Figures $\mathrm{S}-2 \mathrm{~g}, \mathrm{~h}$ ) of the ethylene/ $\mathrm{CH}_{2}=\mathrm{CHO}^{\mathrm{t}} \mathrm{Bu}$ copolymers, which are different from the resonances of the $-\left[\mathrm{CH}_{2} \mathrm{CH}\left(\mathrm{O}^{t} \mathrm{Bu}\right)\right]_{\mathrm{n}^{-}}$homopolymer (Figures S3b,c,e,f). Assignments were made based on the model compound data (S-1, S-2, S-3, Chart S-1) as follows: $\mathrm{CH}_{3} \boldsymbol{C H}\left(\mathrm{O}^{\mathrm{t}} \mathrm{Bu}\right) \mathrm{CH}_{2-}\left({ }^{1} \mathrm{H}\right.$ : $\delta 3.63$, sextet; ${ }^{13} \mathrm{C} \delta 67.1$, confirmed as $\mathrm{CH}$ by DEPT, see $\mathbf{S}-$ 1, Chart S-1), $\boldsymbol{C H}_{3} \mathrm{CH}\left(\mathrm{O}^{t} \mathrm{Bu}\right) \mathrm{CH}_{2^{-}}\left({ }^{1} \mathrm{H}: \delta 1.16, \mathrm{~d} ;{ }^{13} \mathrm{C} \delta 22.8\right.$, confirmed as $\mathrm{CH}_{3}$ by DEPT), $\mathrm{CH}_{3} \mathrm{CH}\left(\mathrm{O}^{\mathrm{t}} \mathrm{Bu}\right) \boldsymbol{C H}_{2^{-}} \quad\left({ }^{1} \mathrm{H}: \delta \quad 1.46, \quad \mathrm{~m} ;{ }^{13} \mathrm{C} \quad \delta \quad 39.0\right.$, confirmed as $\mathrm{CH}_{2}$ by DEPT), $\mathrm{CH}_{2} \boldsymbol{C H}\left(\mathrm{O}^{\mathrm{t}} \mathrm{Bu}\right) \mathrm{CH}_{2^{-}}\left({ }^{1} \mathrm{H}: \delta 3.48\right.$, quintet; ${ }^{13} \mathrm{C} \delta 71.2$, confirmed as $\mathrm{CH}$ by DEPT, see $\mathbf{S - 2}, \mathbf{S}-\mathbf{3}$, Chart S-1), $-\boldsymbol{C H}_{2} \mathrm{CH}\left(\mathrm{O}^{\mathrm{t}} \mathrm{Bu}\right) \mathrm{CH}_{2}-\left({ }^{1} \mathrm{H}: \delta 1.48, \mathrm{~m} ;{ }^{13} \mathrm{C} \delta 36.3\right.$, confirmed as $\mathrm{CH}_{2}$ by DEPT).

Confirmation of assignments by COSY (Figure S-2e): $\mathrm{CH}_{3} \mathrm{CH}\left(\mathrm{O}^{\mathrm{t}} \mathrm{Bu}\right) \mathrm{CH}_{2}$ : the sextet at $\delta$ 3.63 is correlated to the doublet at $\delta 1.16$ and the multiplet at $\delta 1.46 .-\mathrm{CH}_{2} \mathrm{CH}\left(\mathrm{O}^{\mathrm{t}} \mathrm{Bu}\right) \mathrm{CH}_{2}$-: the quintet at $\delta 3.48$ is correlated to the multiplet at $\delta 1.48 .{ }^{14}$

These assignments were further confirmed by comparison to the ethylene $/ \mathrm{CH}_{2}=\mathrm{CHOH}$ copolymer derived from the ethylene $/ \mathrm{CH}_{2}=\mathrm{CHO}^{\mathrm{t}} \mathrm{Bu}$ copolymer (vide infra).

Key NMR Data for Ethylene $/ \mathbf{C H}_{2}=\mathbf{C H O}^{\mathbf{t}} \mathbf{B u}$ Copolymer. ${ }^{1} \mathbf{H} \mathbf{N M R}\left(\mathrm{CDCl}_{2} \mathrm{CDCl}_{2}, 100{ }^{\circ} \mathrm{C}\right.$, NMR500-1, SL309-3): $\delta 5.89$ (m, $\left.\mathrm{CH}_{2}=\mathrm{CHCH}_{2}-\right), 5.48$ (m, $\left.\mathrm{CH}_{3} \mathrm{CH}=\mathrm{CHCH}_{2}-\right), 5.05$ (d, $J=17$, 
$\left.\mathrm{CH} \mathrm{H}^{\prime}=\mathrm{CH}-\right), 4.99\left(\mathrm{~d}, J=10, \mathrm{CH} H^{\prime}=\mathrm{CH}-\right), 3.63$ (sextet, $\left.J=6, \mathrm{CH}_{3} \mathrm{CH}\left(\mathrm{O}^{\mathrm{t}} \mathrm{Bu}\right) \mathrm{CH}_{2}-\right), 3.48$ (quintet, $\left.J=6,-\mathrm{CH}_{2} \mathrm{CH}\left(\mathrm{O}^{\mathrm{t}} \mathrm{Bu}\right) \mathrm{CH}_{2}-\right), 2.12\left(\mathrm{q}, J=7, \mathrm{CH}_{2}=\mathrm{CHCH}_{2} \mathrm{CH}_{2}-\right), 2.10\left(\mathrm{~m}, \mathrm{CH}_{3} \mathrm{CH}=\mathrm{CHCH}_{2} \mathrm{CH}_{2}-\right)$, $1.70\left(\mathrm{~d}, J=6\right.$, trans- $\left.\mathrm{CH}_{3} \mathrm{CH}=\mathrm{CH}-\right), 1.67$ (d, $J=6$, cis- $\left.\mathrm{CH}_{3} \mathrm{CH}=\mathrm{CH}-\right), 1.47\left(\mathrm{~m},-\mathrm{CH}\left(\mathrm{O}^{\mathrm{t}} \mathrm{Bu}\right) \mathrm{CH}_{2}-\right)$, $1.35\left(-\mathrm{CH}_{2} \mathrm{CH}_{2^{-}}\right), 1.23\left(\mathrm{~s}, \mathrm{O}^{\mathrm{t}} \mathrm{Bu}\right), 1.16\left(\mathrm{~d}, J=6, \mathrm{CH}_{3} \mathrm{CH}\left(\mathrm{O}^{\mathrm{t}} \mathrm{Bu}\right) \mathrm{CH}_{2^{-}}\right), 0.96$ (t, $\left.J=7, \mathrm{CH}_{3} \mathrm{CH}_{2^{-}}\right)$, $0.92\left(\mathrm{~d}, J=6,-\mathrm{CH}\left(\mathrm{CH}_{3}\right)-\right) .{ }^{1} \mathrm{H}-{ }^{1} \mathrm{H}$ COSY correlations $\left(\mathrm{CDCl}_{2} \mathrm{CDCl}_{2}, 100{ }^{\circ} \mathrm{C}\right.$, NMR500-1, SL308-10): $\delta / \delta 3.63\left(\mathrm{CH}_{3} \mathrm{CH}\left(\mathrm{O}^{t} \mathrm{Bu}\right) \mathrm{CH}_{2^{-}}\right) / 1.46\left(\mathrm{CH}_{3} \mathrm{CH}\left(\mathrm{O}^{\mathrm{t}} \mathrm{Bu}\right) \mathrm{CH}_{2^{-}}\right), 3.63\left(\mathrm{CH}_{3} \mathrm{CH}\left(\mathrm{O}^{\mathrm{t}} \mathrm{Bu}\right) \mathrm{CH}_{2^{-}}\right.$ )/1.16 $\left.\left(\mathrm{CH}_{3} \mathrm{CH}\left(\mathrm{O}^{\mathrm{t}} \mathrm{Bu}\right) \mathrm{CH}_{2}-\right), 3.48\left(-\mathrm{CH}_{2} \mathrm{CH}\left(\mathrm{O}^{\mathrm{t}} \mathrm{Bu}\right) \mathrm{CH}_{2^{-}}\right) / 1.48\left(-\mathrm{CH}_{2} \mathrm{CH}\left(\mathrm{O}^{\mathrm{t}} \mathrm{Bu}\right) \mathrm{CH}_{2^{-}}\right) .{ }^{13} \mathbf{C}^{1}{ }^{1} \mathbf{H}\right\}$ NMR $\left(\mathrm{CDCl}_{2} \mathrm{CDCl}_{2}, 100{ }^{\circ} \mathrm{C}, \mathrm{NMR} 400-1, \mathrm{SL309-4,5): \delta} 71.2\left(-\mathrm{CH}_{2} \mathrm{CH}\left(\mathrm{O}^{t} \mathrm{Bu}\right) \mathrm{CH}_{2}-\right), 67.1\right.$ $\left(\mathrm{CH}_{3} \mathrm{CH}\left(\mathrm{O}^{t} \mathrm{Bu}\right) \mathrm{CH}_{2}-\right), 39.0\left(\mathrm{CH}_{3} \mathrm{CH}\left(\mathrm{O}^{t} \mathrm{Bu}\right) \mathrm{CH}_{2^{-}}\right), 36.3\left(-\mathrm{CH}_{2} \mathrm{CH}\left(\mathrm{O}^{t} \mathrm{Bu}\right) \mathrm{CH}_{2^{-}}\right), 29.4\left(-\mathrm{CH}_{2} \mathrm{CH}_{2}-\right)$, $25.6\left(-\mathrm{CH}\left(\mathrm{O}^{t} \mathrm{Bu}\right) \mathrm{CH}_{2} \mathrm{CH}_{2}-\right), 25.2\left(-\mathrm{CH}\left(\mathrm{O}^{t} \mathrm{Bu}\right) \mathrm{CH}_{2} \mathrm{CH}_{2}-\right), 22.8\left(\mathrm{CH}_{3} \mathrm{CH}\left(\mathrm{O}^{t} \mathrm{Bu}\right) \mathrm{CH}_{2}-\right)$; assignments confirmed by DEPT.

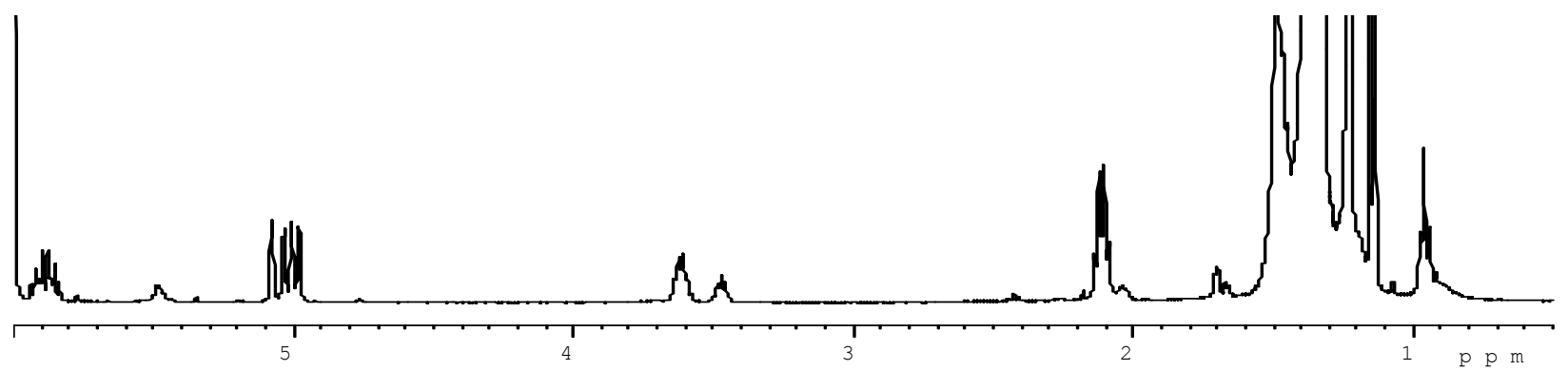

Figure S-2a. ${ }^{1} \mathrm{H}$ NMR $\left(\mathrm{CDCl}_{2} \mathrm{CDCl}_{2}, 100{ }^{\circ} \mathrm{C}\right)$ of ethylene $/ \mathrm{CH}_{2}=\mathrm{CHO}^{\mathrm{t}} \mathrm{Bu}$ copolymer: vertical expansion. The signal at $\delta 6.00$ is from the solvent.

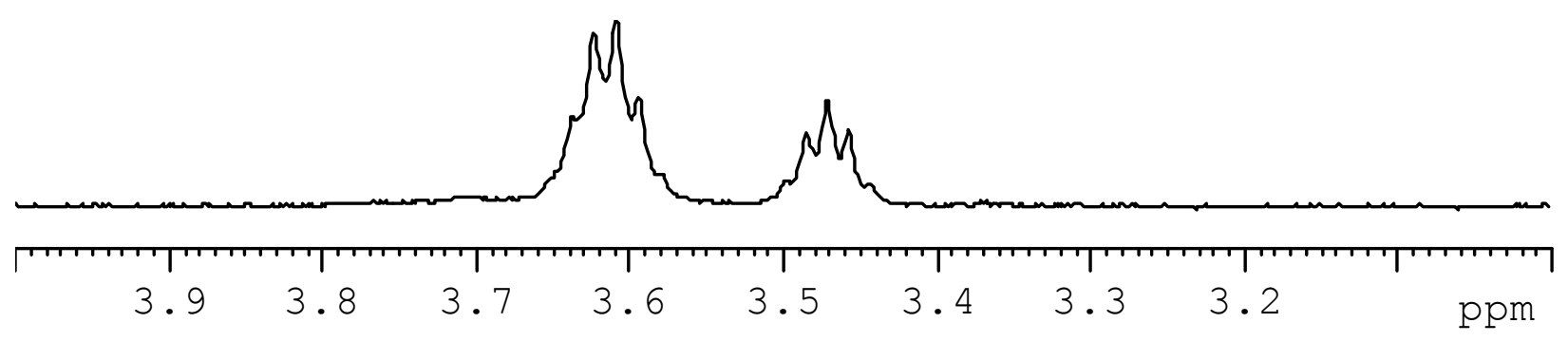


Figure S-2b. ${ }^{1} \mathrm{H}$ NMR $\left(\mathrm{CDCl}_{2} \mathrm{CDCl}_{2}, 100{ }^{\circ} \mathrm{C}\right)$ of ethylene $/ \mathrm{CH}_{2}=\mathrm{CHO}{ }^{\mathrm{t}} \mathrm{Bu}$ copolymer: expansion of the $\delta$ 4.0-3.0 region.

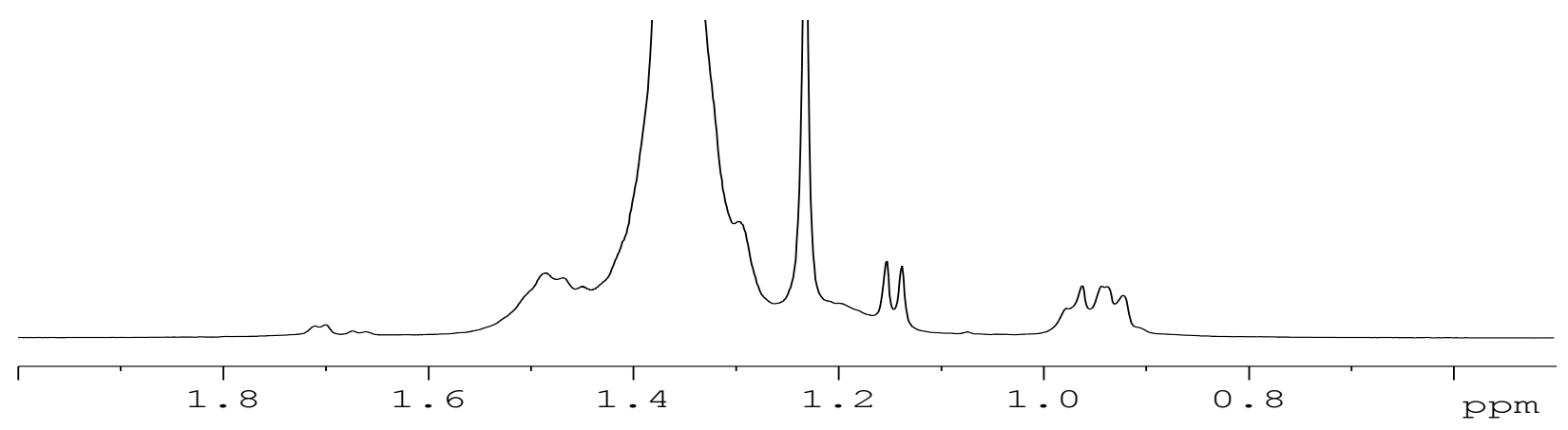

Figure S-2c. ${ }^{1} \mathrm{H}$ NMR $\left(\mathrm{CDCl}_{2} \mathrm{CDCl}_{2}, 100{ }^{\circ} \mathrm{C}\right)$ of ethylene/ $\mathrm{CH}_{2}=\mathrm{CHO}^{\mathrm{t}} \mathrm{Bu}$ copolymer: expansion of the $\delta 2.0-0.5$ region.

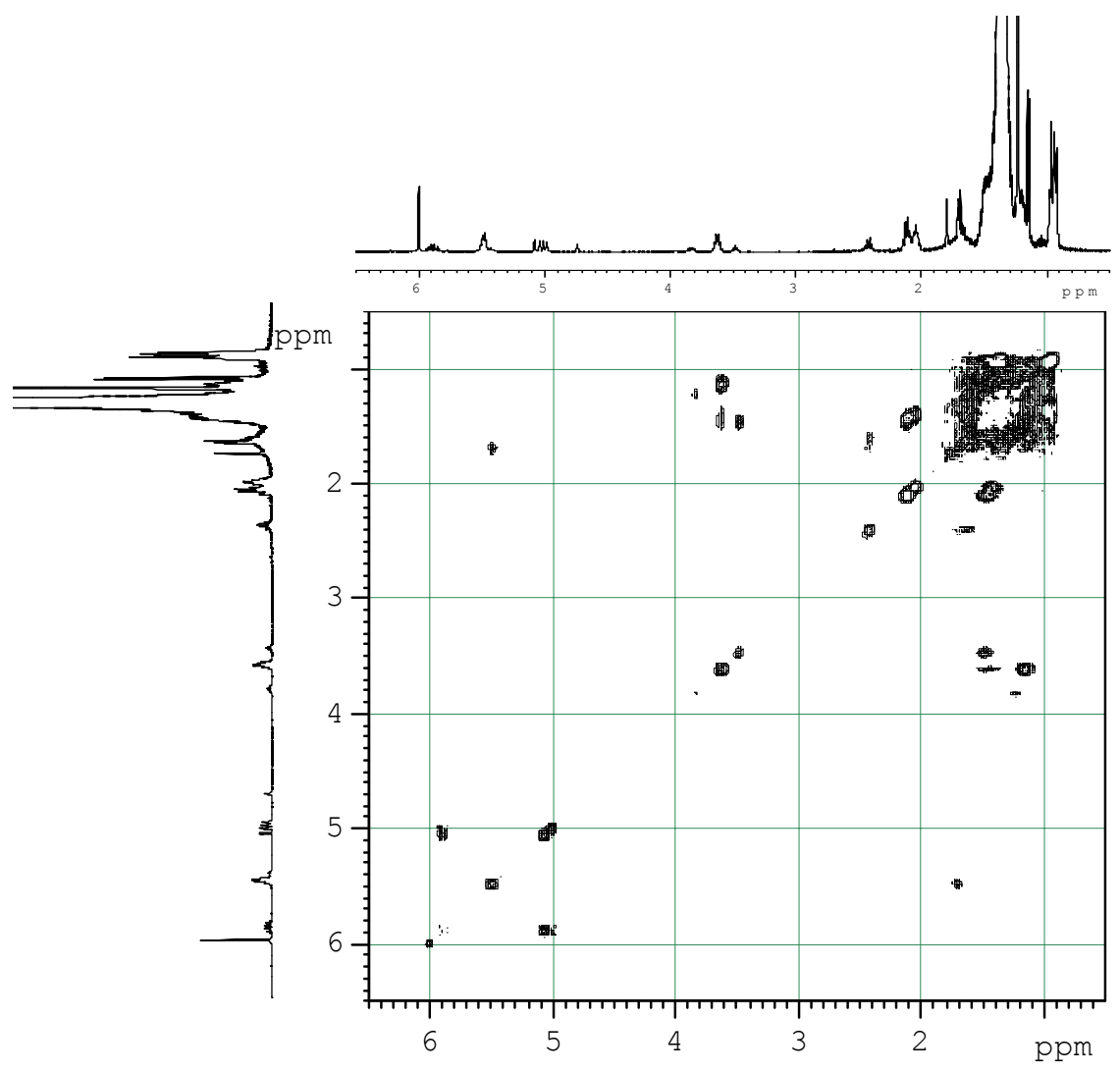

Figure S-2d. COSY NMR $\left(\mathrm{CDCl}_{2} \mathrm{CDCl}_{2}, 100{ }^{\circ} \mathrm{C}\right)$ of ethylene $/ \mathrm{CH}_{2}=\mathrm{CHO}^{\mathrm{t}} \mathrm{Bu}$ copolymer: 
expansion of the $\delta 6.5-0.5 ; 6.5-0.5$ region. The signal at $\delta 3.83$ is from $\mathrm{CH}_{3} \mathrm{CH}(\mathrm{OH}) \mathrm{CH}_{2-}$.

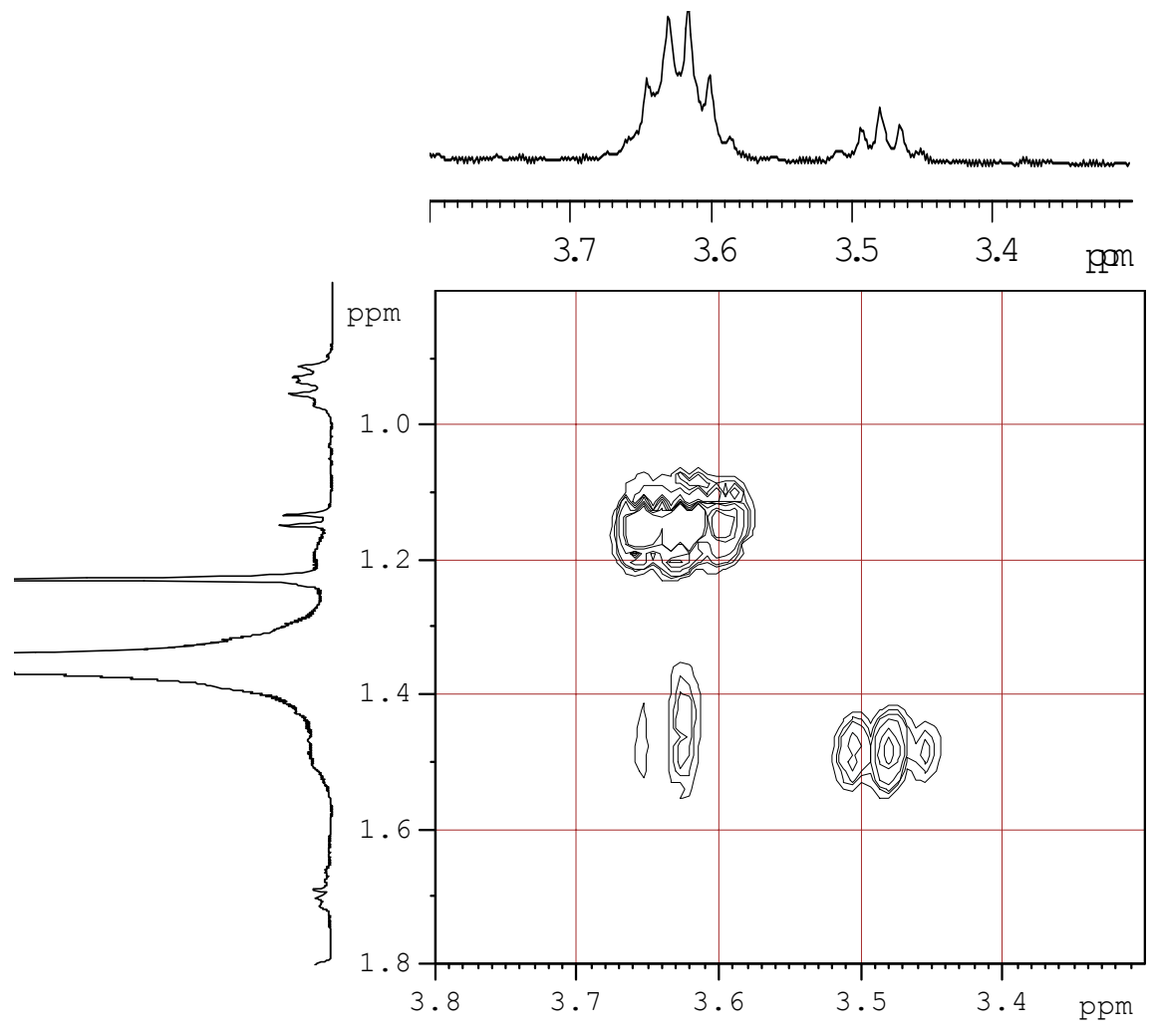

Figure S-2e. COSY NMR $\left(\mathrm{CDCl}_{2} \mathrm{CDCl}_{2}, 100{ }^{\circ} \mathrm{C}\right)$ of ethylene $/ \mathrm{CH}_{2}=\mathrm{CHO}^{\mathrm{t}} \mathrm{Bu}$ copolymer: expansion of the $\delta 3.8-3.3 ; 1.8-0.8$ region.

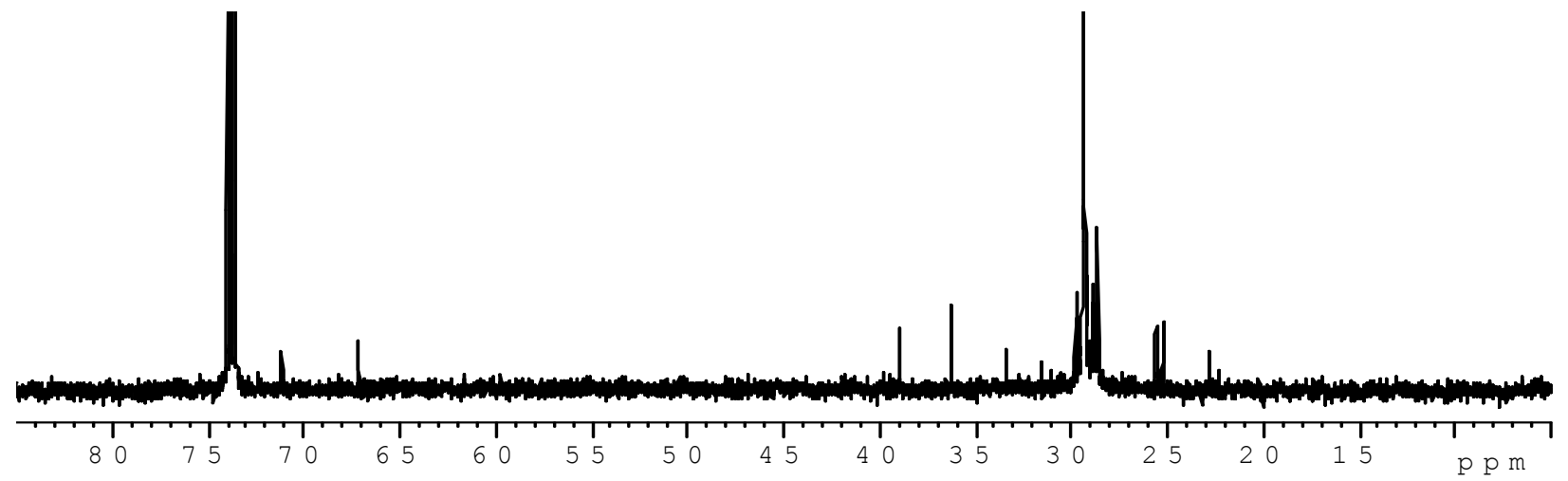


Figure S-2f. ${ }^{13} \mathrm{C} \mathrm{NMR}\left(\mathrm{CDCl}_{2} \mathrm{CDCl}_{2}, 100{ }^{\circ} \mathrm{C}\right)$ of ethylene $/ \mathrm{CH}_{2}=\mathrm{CHO}^{\mathrm{t}} \mathrm{Bu}$ copolymer: expansion of the $\delta 85-5$ region. The signal at $\delta 73.8$ is from the solvent.

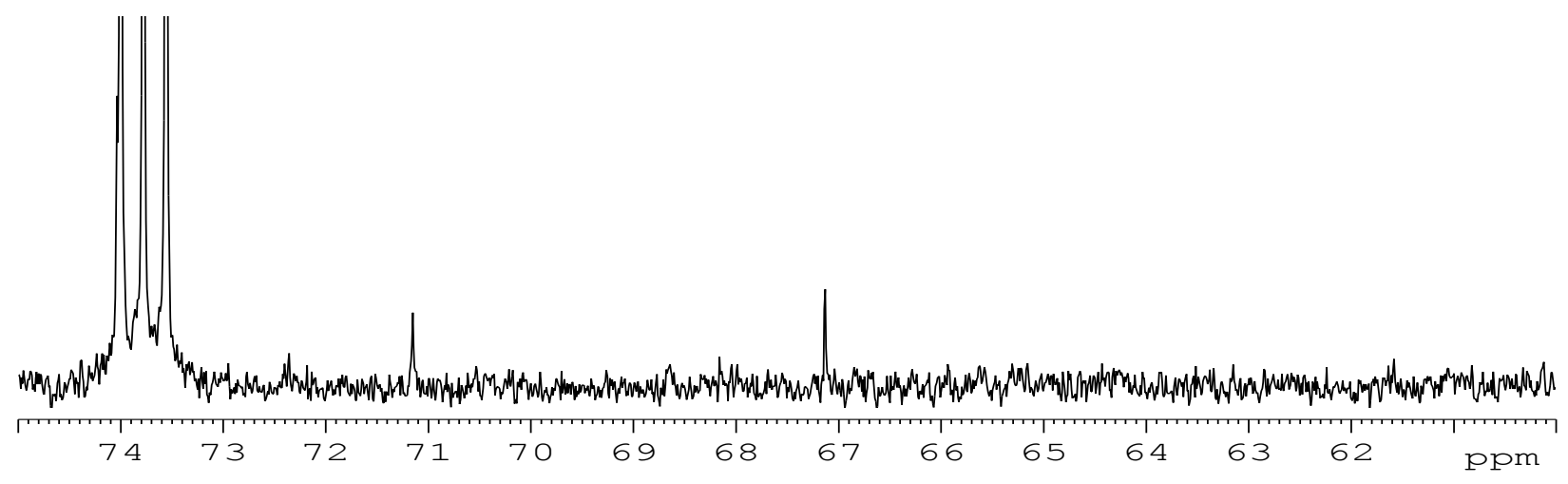

Figure S-2g. ${ }^{13} \mathrm{C} \mathrm{NMR}\left(\mathrm{CDCl}_{2} \mathrm{CDCl}_{2}, 100{ }^{\circ} \mathrm{C}\right)$ of ethylene $/ \mathrm{CH}_{2}=\mathrm{CHO}^{\mathrm{t}} \mathrm{Bu}$ copolymer: expansion of the $\delta 75-60$ region. The signal at $\delta 73.8$ is from the solvent.

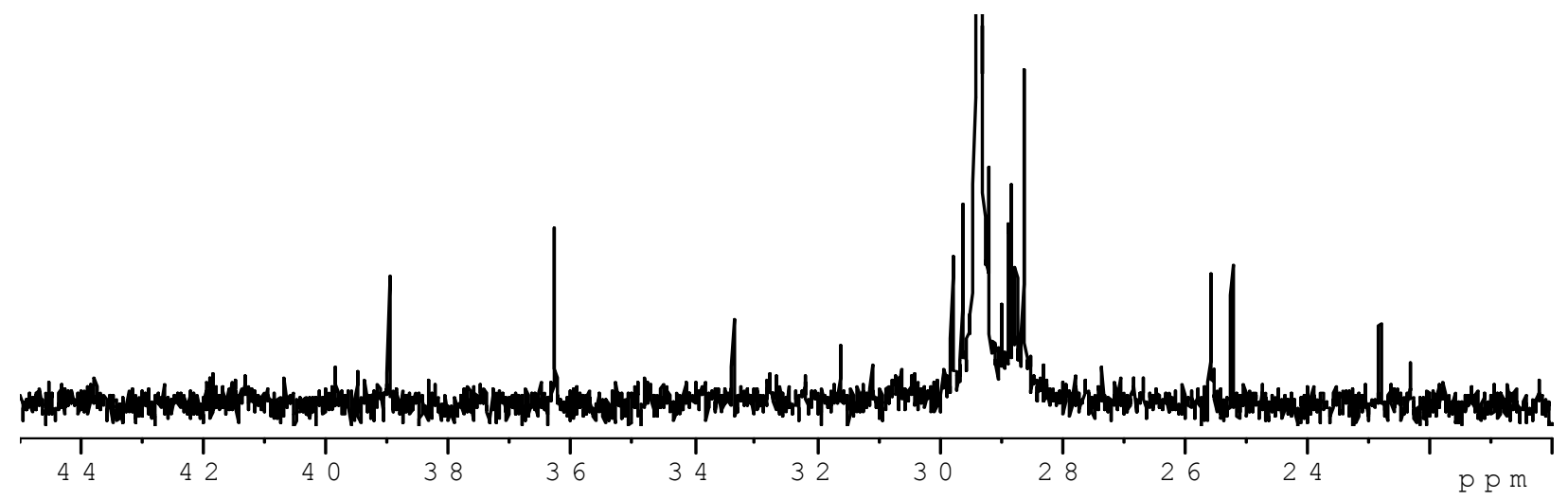

Figure S-2h. ${ }^{13} \mathrm{C} \mathrm{NMR}\left(\mathrm{CDCl}_{2} \mathrm{CDCl}_{2}, 100{ }^{\circ} \mathrm{C}\right)$ of ethylene $/ \mathrm{CH}_{2}=\mathrm{CHO}^{\mathrm{t}} \mathrm{Bu}$ copolymer: expansion of the $\delta 45-20$ region.

Key NMR Data for $-\left[\mathrm{CH}_{2} \mathbf{C H}\left(\mathrm{O}^{t} \mathbf{B u}\right)\right]_{\mathbf{n}^{-}}{ }^{15,16}{ }^{1} \mathbf{H}$ NMR $\left(\mathrm{CDCl}_{2} \mathrm{CDCl}_{2}, 100{ }^{\circ} \mathrm{C}, \mathrm{NMR} 400-1\right.$, polytBuVE-1): $\delta 3.74$ (br, 1H, $\left.-\mathrm{CH}_{2} \mathrm{CH}\left(\mathrm{O}^{\mathrm{t}} \mathrm{Bu}\right)-\right), 1.75$ (br, 2H, $\left.-\mathrm{CH}_{2} \mathrm{CH}\left(\mathrm{O}^{\mathrm{t}} \mathrm{Bu}\right)-\right), 1.29$ (br, 9H, $\left.\left.\mathrm{CH}_{2} \mathrm{CH}(\mathrm{OCMe})_{3}\right)-\right) .{ }^{13} \mathrm{C}$ NMR $\left(\mathrm{CDCl}_{2} \mathrm{CDCl}_{2}, 100{ }^{\circ} \mathrm{C}, \mathrm{NMR} 400-1\right.$, polytBuVE-2): $\delta 72.8(-$ $\left.\mathrm{CH}_{2} \mathrm{CH}\left(\mathrm{OCMe}_{3}\right)-\right), 68.0$ (br, mm, $\left.-\mathrm{CH}_{2} \mathrm{CH}\left(\mathrm{O}^{t} \mathrm{Bu}\right)-\right), 67.4$ (br, mr, $\left.-\mathrm{CH}_{2} \mathrm{CH}\left(\mathrm{O}^{t} \mathrm{Bu}\right)-\right)$, 66.7 (br, rr, $\left.\mathrm{CH}_{2} \mathrm{CH}\left(\mathrm{O}^{\mathrm{t}} \mathrm{Bu}\right)-\right), \quad 45.6$ (br, $\left.\quad-\mathrm{CH}_{2} \mathrm{CH}\left(\mathrm{O}^{\mathrm{t}} \mathrm{Bu}\right)-\right), 29.6$ (br, $\left.-\mathrm{CH}_{2} \mathrm{CH}\left(\mathrm{OCMe}_{3}\right)-\right)$. The - 
$\left[\mathrm{CH}_{2} \mathrm{CH}\left(\mathrm{O}^{\mathrm{t}} \mathrm{Bu}\right)\right]_{\mathrm{n}}$ - homopolymer reacts to generate isobutylene (6\%) in $\mathrm{CDCl}_{2} \mathrm{CDCl}_{2}$ at $100{ }^{\circ} \mathrm{C}$ in $50 \mathrm{~min}$.

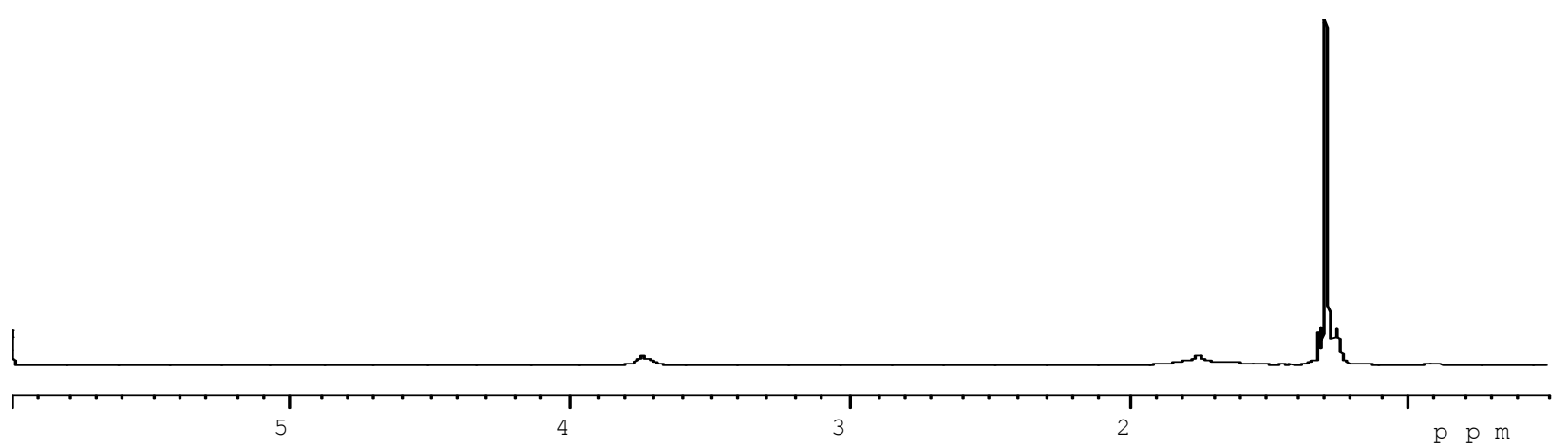

Figure S-3a. ${ }^{1} \mathrm{H}$ NMR $\left(\mathrm{CDCl}_{2} \mathrm{CDCl}_{2}, 100{ }^{\circ} \mathrm{C}\right)$ of $-\left[\mathrm{CH}_{2}=\mathrm{CH}\left(\mathrm{O}^{\mathrm{t}} \mathrm{Bu}\right)\right]_{\mathrm{n}}$ - polymer: expansion of the $\delta 6.0-0.5$ region. The signal at $\delta 6.00$ is from the solvent.

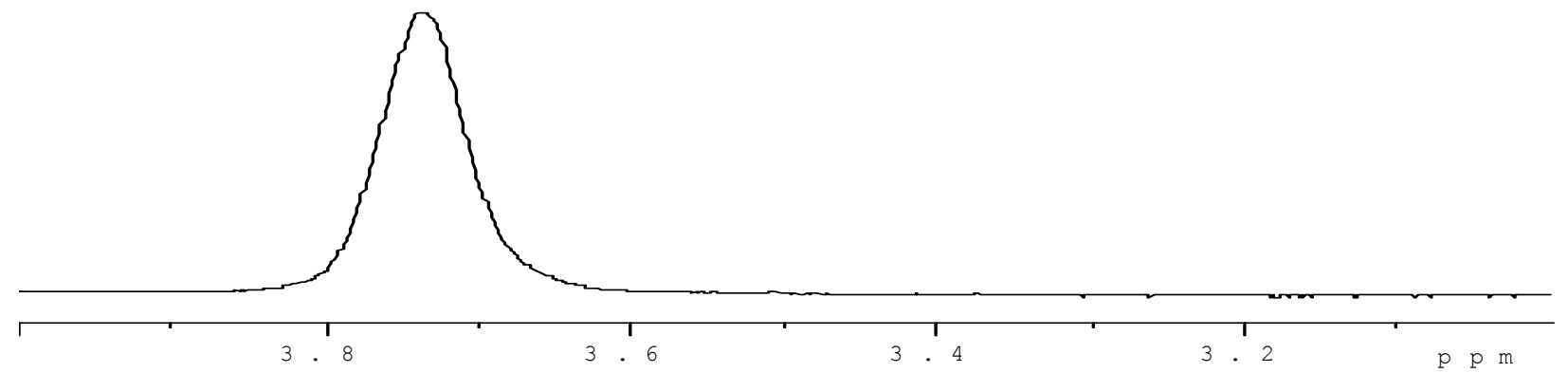

Figure S-3b. ${ }^{1} \mathrm{H}$ NMR $\left(\mathrm{CDCl}_{2} \mathrm{CDCl}_{2}, 100{ }^{\circ} \mathrm{C}\right)$ of $-\left[\mathrm{CH}_{2}=\mathrm{CH}\left(\mathrm{O}^{\mathrm{t}} \mathrm{Bu}\right)\right]_{\mathrm{n}}$ - polymer: expansion of the $\delta$ 4.0-3.0 region.

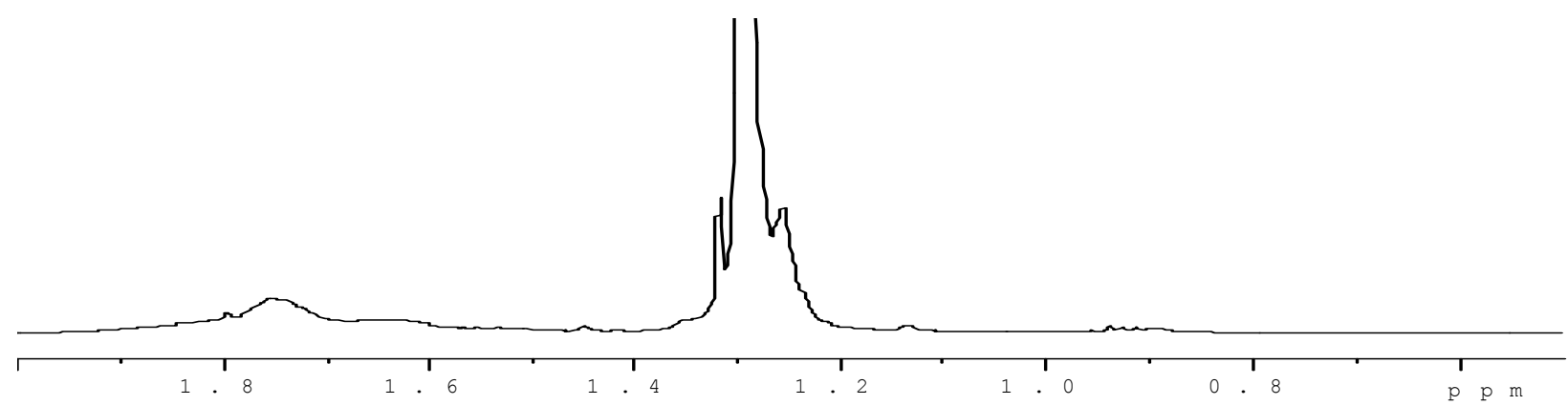


Figure S-3c. ${ }^{1} \mathrm{H} \mathrm{NMR}\left(\mathrm{CDCl}_{2} \mathrm{CDCl}_{2}, 100{ }^{\circ} \mathrm{C}\right)$ of $-\left[\mathrm{CH}_{2}=\mathrm{CH}\left(\mathrm{O}^{\mathrm{t}} \mathrm{Bu}\right)\right]_{\mathrm{n}}$ - polymer: expansion of the $\delta$ 2.0-0.5 region.

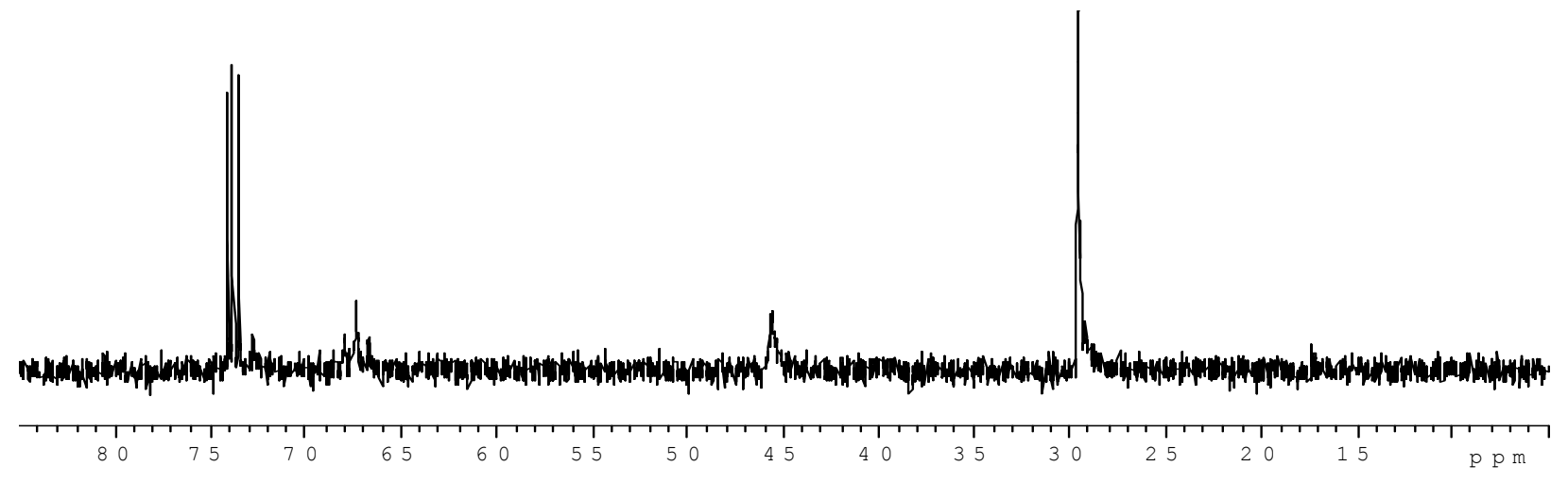

Figure S-3d. ${ }^{13} \mathrm{C}$ NMR $\left(\mathrm{CDCl}_{2} \mathrm{CDCl}_{2}, 100{ }^{\circ} \mathrm{C}\right)$ of $-\left[\mathrm{CH}_{2}=\mathrm{CH}\left(\mathrm{O}^{\mathrm{t}} \mathrm{Bu}\right)\right]_{\mathrm{n}}$ - polymer: expansion of the $\delta 85-5$ region. The signal at $\delta 73.8$ is from the solvent.

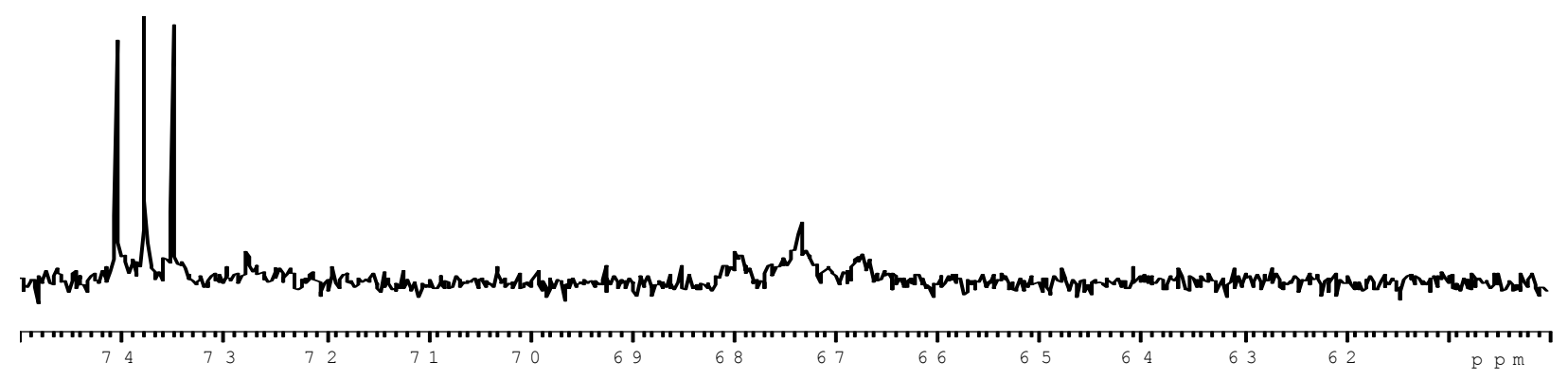

Figure S-3e. ${ }^{1} \mathrm{H} \mathrm{NMR}\left(\mathrm{CDCl}_{2} \mathrm{CDCl}_{2}, 100{ }^{\circ} \mathrm{C}\right)$ of $-\left[\mathrm{CH}_{2}=\mathrm{CH}\left(\mathrm{O}^{\mathrm{t}} \mathrm{Bu}\right)\right]_{\mathrm{n}}$ - polymer: expansion of the $\delta 75-60$ region. The signal at $\delta 73.8$ is from the solvent.

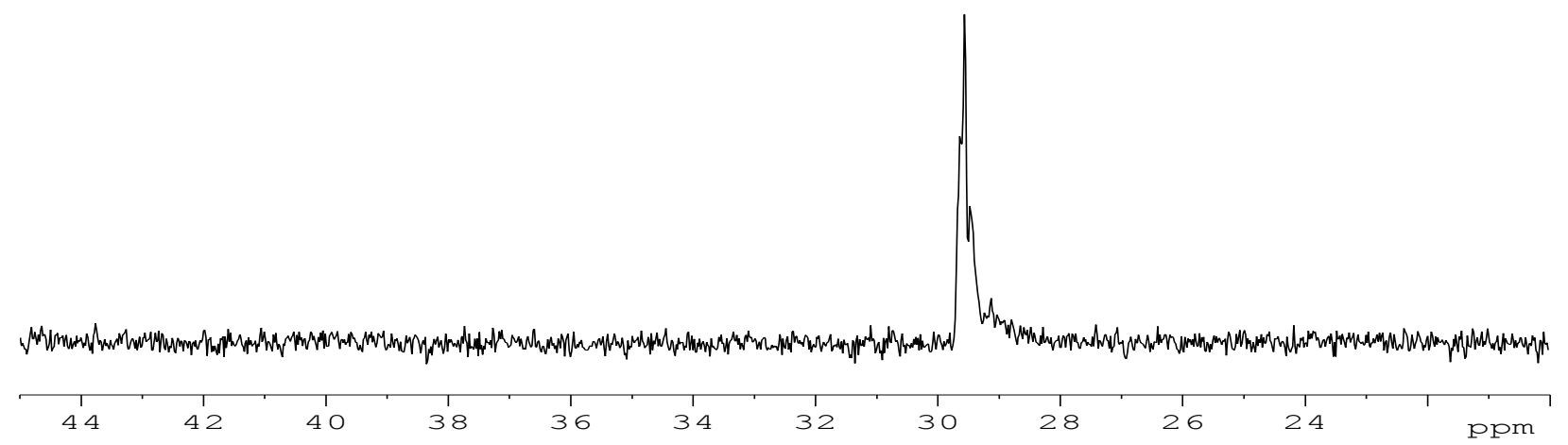


Figure S-3f. ${ }^{1} \mathrm{H}$ NMR $\left(\mathrm{CDCl}_{2} \mathrm{CDCl}_{2}, 100{ }^{\circ} \mathrm{C}\right)$ of $-\left[\mathrm{CH}_{2}=\mathrm{CH}\left(\mathrm{O}^{\mathrm{t}} \mathrm{Bu}\right)\right]_{\mathrm{n}}$ - polymer: expansion of the $\delta$ 45-20 region.

\section{Thermal Conversion of Ethylene $/ \mathrm{CH}_{2}=\mathrm{CHO}^{\mathrm{t}} \mathrm{Bu}$ Copolymer to Ethylene $/ \mathrm{CH}_{2}=\mathrm{CHOH}$}

Copolymer. An NMR tube was charged with the ethylene $/ \mathrm{CH}_{2}=\mathrm{CHO}^{\mathrm{t}} \mathrm{Bu}$ copolymer (20 mg, 1.2 mol\% $\left.\mathrm{CH}_{2}=\mathrm{CHO}^{\mathrm{t}} \mathrm{Bu}\right)$ and $\mathrm{CDCl}_{2} \mathrm{CDCl}_{2}(0.4 \mathrm{~mL})$. NMR analysis showed that the copolymer contained $\mathrm{CH}_{3} \mathrm{CH}\left(\mathrm{O}^{t} \mathrm{Bu}\right) \mathrm{CH}_{2^{-}}(0.96 \mathrm{~mol} \%),-\mathrm{CH}_{2} \mathrm{CH}\left(\mathrm{O}^{\mathrm{t}} \mathrm{Bu}\right) \mathrm{CH}_{2^{-}}(0.24 \mathrm{~mol} \%),-\mathrm{CH}_{2} \mathrm{CH}^{-} \mathrm{CH}_{2}$ $(1.0 \mathrm{~mol} \%)$ and $-\mathrm{CH}_{2} \mathrm{CH}=\mathrm{CHMe}(0.33 \mathrm{~mol} \%)$. The tube was heated to $120{ }^{\circ} \mathrm{C}$ for $48 \mathrm{~h}$. NMR analysis showed that the copolymer contained $\mathrm{CH}_{3} \mathrm{CH}(\mathrm{OH}) \mathrm{CH}_{2^{-}} \quad(0.99 \mathrm{~mol} \%)$, $\mathrm{CH}_{2} \mathrm{CH}(\mathrm{OH}) \mathrm{CH}_{2}-(0.48 \mathrm{~mol} \%),-\mathrm{CH}_{2} \mathrm{CH}=\mathrm{CH}_{2}(0.28 \mathrm{~mol} \%)$ and $-\mathrm{CH}_{2} \mathrm{CH}=\mathrm{CHMe}(0.83 \mathrm{~mol} \%)$. Therefore, the $\mathrm{CH}_{3} \mathrm{CH}\left(\mathrm{O}^{t} \mathrm{Bu}\right) \mathrm{CH}_{2}-$ and $-\mathrm{CH}_{2} \mathrm{CH}\left(\mathrm{O}^{t} \mathrm{Bu}\right) \mathrm{CH}_{2^{-}}$groups were converted to $\mathrm{CH}_{3} \mathrm{CH}(\mathrm{OH}) \mathrm{CH}_{2}$ - and $-\mathrm{CH}_{2} \mathrm{CH}(\mathrm{OH}) \mathrm{CH}_{2}$ - groups and isobutene. ${ }^{17}$ These structural units were identified by comparison of NMR data to data for model compounds (S-1, S-2, S-3, Chart S-1) and by COSY experiments. In addition, some vinyl groups $\left(-\mathrm{CH}_{2} \mathrm{CH}=\mathrm{CH}_{2}\right)$ were converted to 2olefins $\left(-\mathrm{CH}_{2} \mathrm{CH}=\mathrm{CHMe}\right)$. Key NMR Data for ethylene $/ \mathrm{CH}_{2}=\mathrm{CHOH}$ copolymer: ${ }^{1} \mathbf{H}$ NMR $\left(\mathrm{CDCl}_{2} \mathrm{CDCl}_{2}, 100{ }^{\circ} \mathrm{C}, \mathrm{NMR} 400-2, \mathrm{SL} 308-15\right): \delta 3.82$ (sextet, $\left.J=6, \mathrm{CH}_{3} \mathrm{CH}(\mathrm{OH}) \mathrm{CH}_{2}-\right), 3.63$ (quintet, $\left.J=5,-\mathrm{CH}_{2} \mathrm{CH}(\mathrm{OH}) \mathrm{CH}_{2}-\right), 1.69(\mathrm{~s}, \mathrm{OH}), 1.52\left(\mathrm{~m}, \mathrm{CH}_{3} \mathrm{CH}(\mathrm{OH}) \mathrm{CH}_{2^{-}}\right), 1.46(\mathrm{~m}$, $\left.\mathrm{CH}_{2} \mathrm{CH}(\mathrm{OH}) \mathrm{CH}_{2^{-}}\right), 1.23\left(\mathrm{~d}, \mathrm{~J}=6, \mathrm{CH}_{3} \mathrm{CH}(\mathrm{OH}) \mathrm{CH}_{2^{-}}\right) .{ }^{1} \mathrm{H}-{ }^{1} \mathrm{H}$ COSY correlations $\left(\mathrm{CDCl}_{2} \mathrm{CDCl}_{2}\right.$, $100{ }^{\circ} \mathrm{C}, \mathrm{NMR} 500-1, \mathrm{SL308-10)}: \delta / \delta 3.82\left(\mathrm{CH}_{3} \mathrm{CH}(\mathrm{OH}) \mathrm{CH}_{2^{-}}\right) / 1.52\left(\mathrm{CH}_{3} \mathrm{CH}(\mathrm{OH}) \mathrm{CH}_{2^{-}}\right), 3.82$ $\left.\left(\mathrm{CH}_{3} \mathrm{CH}(\mathrm{OH}) \mathrm{CH}_{2^{-}}\right) / 1.23 \quad\left(\mathrm{CH}_{3} \mathrm{CH}(\mathrm{OH}) \mathrm{CH}_{2}\right)^{-}\right), \quad 3.63 \quad\left(-\mathrm{CH}_{2} \mathrm{CH}(\mathrm{OH}) \mathrm{CH}_{2^{-}}\right) / 1.46 \quad(-$ $\left.\mathrm{CH}_{2} \mathrm{CH}(\mathrm{OH}) \mathrm{CH}_{2}-\right) .{ }^{18}{ }^{13} \mathbf{C}\left\{{ }^{1} \mathbf{H}\right\}$ NMR $\left(\mathrm{CDCl}_{2} \mathrm{CDCl}_{2}, 100{ }^{\circ} \mathrm{C}, \mathrm{NMR} 500-1, \mathrm{SL308-5}\right): \delta 71.9(-$ $\left.\mathrm{CH}_{2} \mathrm{CH}(\mathrm{OH}) \mathrm{CH}_{2-}\right), 67.9\left(\mathrm{CH}_{3} \mathrm{CH}(\mathrm{OH}) \mathrm{CH}_{2}-\right), 39.3\left(\mathrm{CH}_{3} \mathrm{CH}(\mathrm{OH}) \mathrm{CH}_{2^{-}}\right), 37.4\left(-\mathrm{CH}_{2} \mathrm{CH}(\mathrm{OH}) \mathrm{CH}_{2^{-}}\right.$ ), $25.4\left(\mathrm{CH}_{3} \mathrm{CH}(\mathrm{OH}) \mathrm{CH}_{2} \mathrm{CH}_{2}-\right), 24.9\left(-\mathrm{CH}_{2} \mathrm{CH}_{2} \mathrm{CH}(\mathrm{OH}) \mathrm{CH}_{2} \mathrm{CH}_{2^{-}}\right), 23.2\left(\mathrm{CH}_{3} \mathrm{CH}(\mathrm{OH}) \mathrm{CH}_{2^{-}}\right)$.

Conversion of Ethylene $/ \mathrm{CH}_{2}=\mathrm{CHO}^{\mathrm{t}} \mathrm{Bu}$ Copolymer to Ethylene $/ \mathrm{CH}_{2}=\mathrm{CHOH}$ Copolymer by Reaction with HCl. (NB3-P43) An NMR tube was charged with the ethylene $/ \mathrm{CH}_{2}=\mathrm{CHO}^{t} \mathrm{Bu}$ copolymer $\left(20 \mathrm{mg}, 1.2 \mathrm{~mol} \% \mathrm{CH}_{2}=\mathrm{CHO}^{\mathrm{t}} \mathrm{Bu}\right)$ and $\mathrm{CDCl}_{2} \mathrm{CDCl}_{2}(0.4 \mathrm{~mL})$. NMR analysis showed that the copolymer contained $\mathrm{CH}_{3} \mathrm{CH}\left(\mathrm{O}^{\mathrm{t}} \mathrm{Bu}\right) \mathrm{CH}_{2^{-}}(0.96 \mathrm{~mol} \%)$, $\mathrm{CH}_{2} \mathrm{CH}\left(\mathrm{O}^{\mathrm{t}} \mathrm{Bu}\right) \mathrm{CH}_{2}-(0.24 \mathrm{~mol} \%),-\mathrm{CH}_{2} \mathrm{CH}=\mathrm{CH}_{2}(1.0 \mathrm{~mol} \%)$ and $-\mathrm{CH}_{2} \mathrm{CH}=\mathrm{CHMe}(0.33 \mathrm{~mol} \%)$. 
$\mathrm{HCl}(0.10 \mathrm{mmol})$ was added by vacuum transfer at $-196{ }^{\circ} \mathrm{C}$. The tube was warmed to $23{ }^{\circ} \mathrm{C}$ and heated to $100{ }^{\circ} \mathrm{C}$ for $24 \mathrm{~h}$. NMR analysis showed that the copolymer contained $\mathrm{CH}_{3} \mathrm{CH}(\mathrm{Cl}) \mathrm{CH}_{2}-$ (0.22 mol\%), identified by comparison to NMR data for model compounds (Chart S-1), $\mathrm{CH}_{3} \mathrm{CH}(\mathrm{OH}) \mathrm{CH}_{2}-(0.96 \mathrm{~mol} \%),-\mathrm{CH}_{2} \mathrm{CH}(\mathrm{OH}) \mathrm{CH}_{2}-(0.25 \mathrm{~mol} \%),-\mathrm{CH}_{2} \mathrm{CH}=\mathrm{CH}_{2}(0.72 \mathrm{~mol} \%)$ and $-\mathrm{CH}_{2} \mathrm{CH}=\mathrm{CHMe}(0.33 \mathrm{~mol} \%)$. Therefore, the $\mathrm{CH}_{3} \mathrm{CH}\left(\mathrm{O}^{\mathrm{t}} \mathrm{Bu}\right) \mathrm{CH}_{2}-$ and $-\mathrm{CH}_{2} \mathrm{CH}\left(\mathrm{O}^{\mathrm{t}} \mathrm{Bu}\right) \mathrm{CH}_{2}-$ groups were converted to $\mathrm{CH}_{3} \mathrm{CH}(\mathrm{OH}) \mathrm{CH}_{2}$ - and $-\mathrm{CH}_{2} \mathrm{CH}(\mathrm{OH}) \mathrm{CH}_{2}$ - and ${ }^{\mathrm{t}} \mathrm{BuCl} .{ }^{19} \mathrm{In}$ addition, vinyl groups $\left(-\mathrm{CH}_{2} \mathrm{CH}=\mathrm{CH}_{2}\right)$ were converted to $\mathrm{CH}_{3} \mathrm{CH}(\mathrm{Cl}) \mathrm{CH}_{2}$ - groups. ${ }^{20}$ The NMR spectra of the $\mathrm{OH}$-copolymer are the same as above.

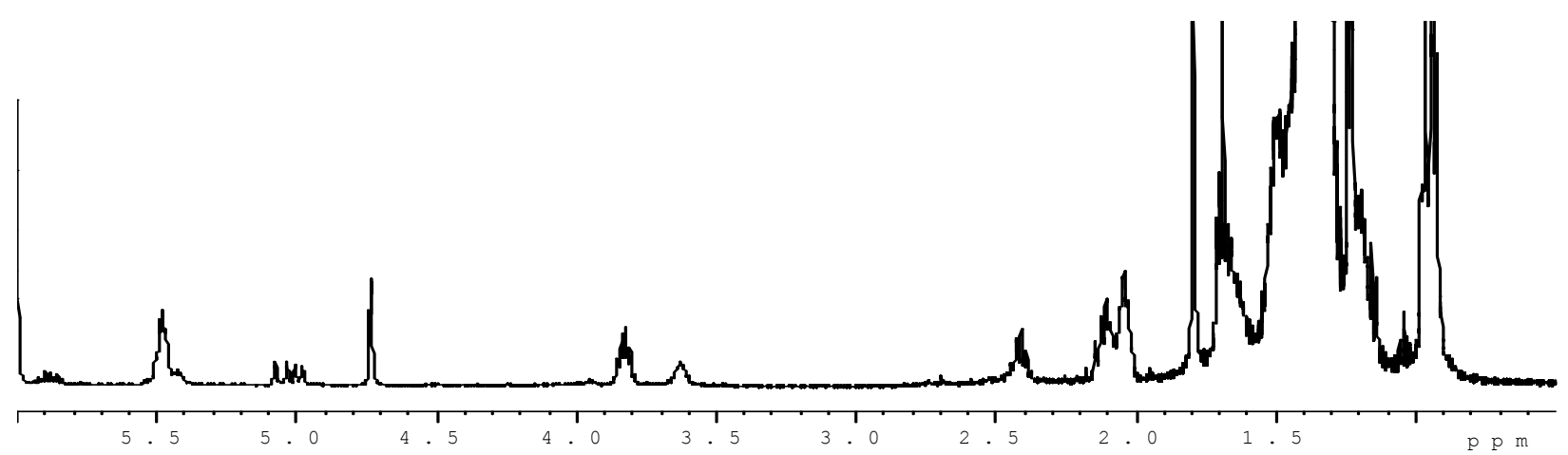

Figure S-4a. ${ }^{1} \mathrm{H} \mathrm{NMR}\left(\mathrm{CDCl}_{2} \mathrm{CDCl}_{2}, 100{ }^{\circ} \mathrm{C}\right)$ of ethylene $/ \mathrm{CH}_{2}=\mathrm{CHOH}$ copolymer: vertical expansion. The signal at $\delta 6.00$ is from the solvent. The signals at $\delta 4.74$ and 1.80 are from isobutene.

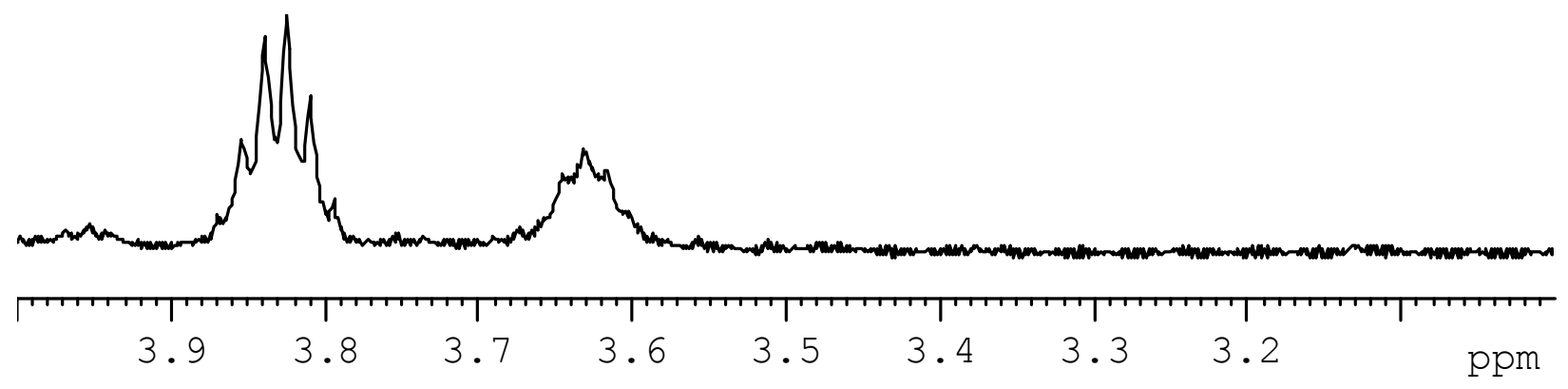


Figure S-4b. ${ }^{1} \mathrm{H}$ NMR $\left(\mathrm{CDCl}_{2} \mathrm{CDCl}_{2}, 100{ }^{\circ} \mathrm{C}\right)$ of ethylene $/ \mathrm{CH}_{2}=\mathrm{CHOH}$ copolymer: expansion of the $\delta$ 4.0-3.0 region.

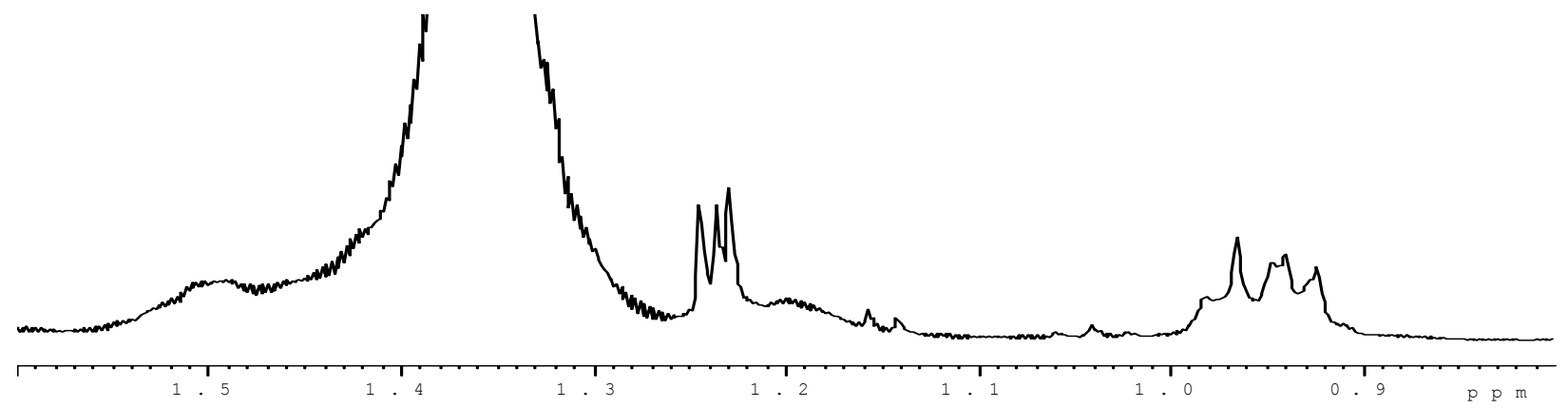

Figure S-4c. ${ }^{1} \mathrm{H}$ NMR $\left(\mathrm{CDCl}_{2} \mathrm{CDCl}_{2}, 100{ }^{\circ} \mathrm{C}\right)$ of ethylene/ $\mathrm{CH}_{2}=\mathrm{CHOH}$ copolymer: expansion of the $\delta 1.6-0.8$ region. The singlet at $\delta 1.23$ is probably from $\mathrm{HO}^{\mathrm{t}} \mathrm{Bu}$.

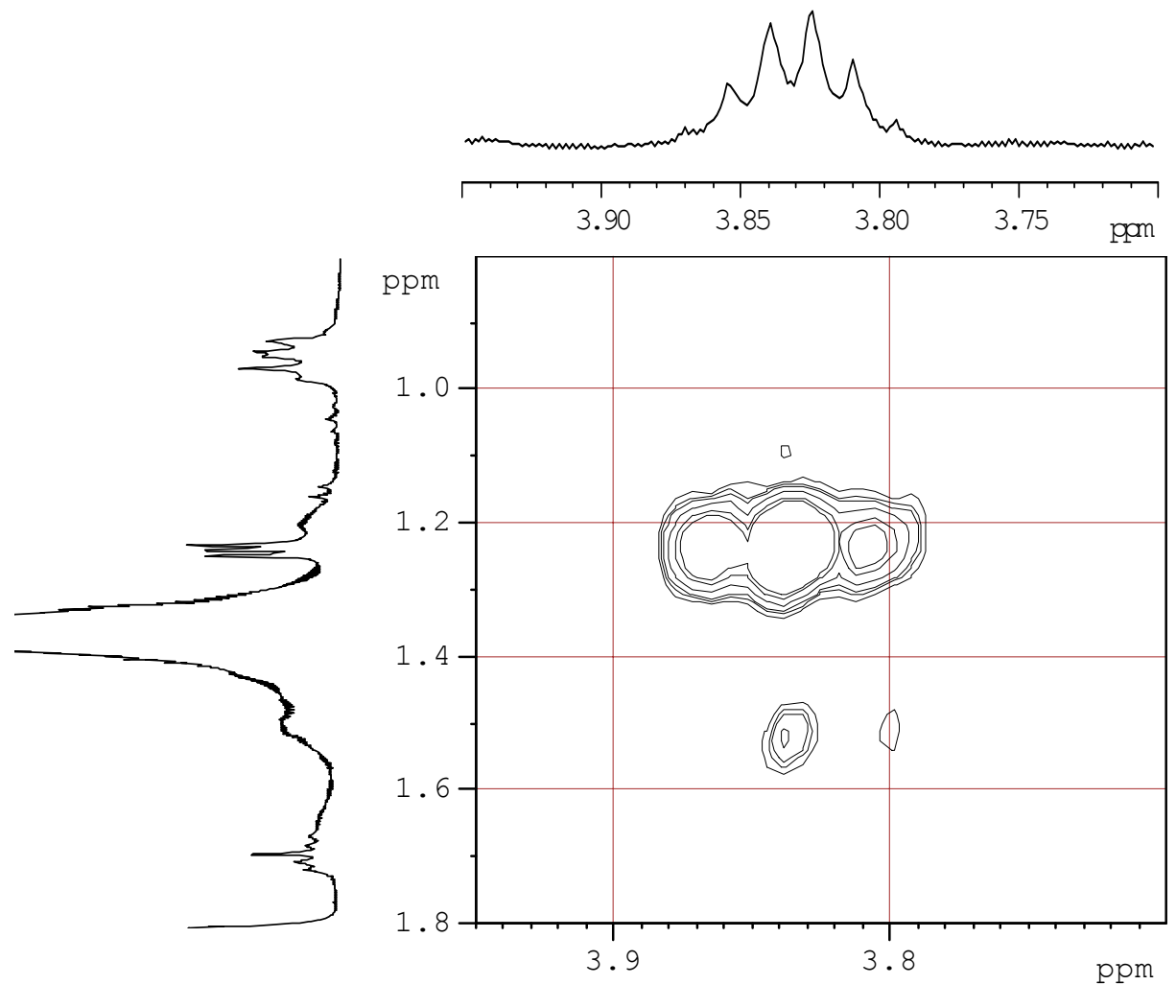

Figure S-4d. COSY NMR $\left(\mathrm{CDCl}_{2} \mathrm{CDCl}_{2}, 100{ }^{\circ} \mathrm{C}\right)$ of ethylene $/ \mathrm{CH}_{2}=\mathrm{CHOH}$ copolymer: expansion of the $\delta 3.95-3.70 ; 1.8-0.8$ region. 
NMR Characterization of Ethylene $/ \mathbf{C H}_{2}=\mathbf{C H O E t}$ Copolymer. The poly(ethylene-co$\mathrm{CH}_{2}=\mathrm{CHOEt}$ ) produced above is very similar to the polyethylene produced in the comparative ethylene homopolymerization described above. NMR studies show that the copolymer contains 2-10 branches/1000 C, mainly methyl branches. The unsaturated units are vinyl groups ($\left.\mathrm{CH}_{2} \mathrm{CH}=\mathrm{CH}_{2}\right)$ and 2-olefins $\left(-\mathrm{CH}_{2} \mathrm{CH}=\mathrm{CHMe}\right){ }^{21}$ Additionally, the copolymer contains two major comonomer units, $\mathrm{CH}_{3} \mathrm{CH}(\mathrm{OEt}) \mathrm{CH}_{2}$ - and $-\mathrm{CH}_{2} \mathrm{CH}(\mathrm{OEt}) \mathrm{CH}_{2}$, which have been identified by 1- and 2-D NMR and comparison with data for model compounds (S-4, S-5, S-6, Chart S-1). A detailed discussion of how these assignments were made is provided in the next section. Representative 1- and 2-D NMR spectra of the copolymer are shown in Figures S-5a-n. The assignments for major comonomer units are listed below. Data for model compounds are listed in Chart S-1 in the General Information section. All other resonances in the spectra of the copolymer are present in the spectra of homo-polyethylene. Resonances for the homopolymer $\left[\mathrm{CH}_{2} \mathrm{CH}(\mathrm{OEt})\right]_{\mathrm{n}}$ - were not observed in the spectra of poly (ethylene-co- $\left.\mathrm{CH}_{2}=\mathrm{CHOEt}\right){ }^{22}$

NMR Assignments of Key Comonomer Units in Ethylene $/ \mathrm{CH}_{2}=\mathrm{CHOEt}$ Copolymer. Resonances for $\boldsymbol{- C H}(\mathrm{OEt})$ - comonomer units were found in the ${ }^{1} \mathrm{H}$ NMR spectra (Figures S-5b,c) and the ${ }^{13} \mathrm{C}$ NMR spectra (Figure S-5h,i) of the ethylene $/ \mathrm{CH}_{2}=\mathrm{CHOEt}$ copolymers, which are different from the resonances of the $-\left[\mathrm{CH}_{2} \mathrm{CH}(\mathrm{OEt})\right]_{\mathrm{n}}$ - homopolymer. ${ }^{22} \mathrm{HMQC}$ spectra (Figures $\mathrm{S}-5 \mathrm{j}, \mathrm{k})$ established the $\mathrm{C}-\mathrm{H}$ correlation for the $\boldsymbol{- C H}(\mathrm{OEt})$ - units. Assignments were made based on the HMQC data and model compound data (S-4, S-5, S-6, Chart S-1) as follows: $\mathrm{CH}_{3} \boldsymbol{C H}(\mathrm{OEt}) \mathrm{CH}_{2}-\left({ }^{1} \mathrm{H} \delta 3.40, \mathrm{~m} ;{ }^{13} \mathrm{C} \delta 74.8\right.$, confirmed as $\mathrm{CH}$ by DEPT, see $\mathbf{S}-\mathbf{4}$, Chart S-1), $\boldsymbol{C H}_{3} \mathrm{CH}(\mathrm{OEt}) \mathrm{CH}_{2-}\left({ }^{1} \mathrm{H} \delta 1.15, \mathrm{~d} ;{ }^{13} \mathrm{C} \delta 19.8\right.$, confirmed as $\mathrm{CH}_{3}$ by DEPT $), \mathrm{CH}_{3} \mathrm{CH}(\mathrm{OEt}) \boldsymbol{C H}_{2^{-}}$ $\left({ }^{1} \mathrm{H} \delta 1.55, \mathrm{~m} ;{ }^{13} \mathrm{C} \delta 36.7\right.$, confirmed as $\mathrm{CH}_{2}$ by DEPT), $-\mathrm{CH}_{2} \boldsymbol{C H}(\mathrm{OEt}) \mathrm{CH}_{2}-\left({ }^{1} \mathrm{H} \delta 3.23\right.$, quintet; ${ }^{13} \mathrm{C} \delta 79.1$, confirmed as CH by DEPT, see $\mathbf{S - 5}, \mathbf{S - 6}$, Chart $\left.\mathrm{S}-1\right),-\boldsymbol{C H}_{2} \mathrm{CH}(\mathrm{OEt}) \mathrm{CH}_{2}-\left({ }^{1} \mathrm{H} \delta 1.50\right.$, $\mathrm{m} ;{ }^{13} \mathrm{C} \delta 34.2$, confirmed as $\mathrm{CH}_{2}$ by DEPT), $-\mathrm{CH}\left(\mathrm{OCH}_{2} \mathrm{CH}_{3}\right) \mathrm{CH}_{2}-\left({ }^{1} \mathrm{H} \delta 3.50, \mathrm{q} ;{ }^{13} \mathrm{C} \delta 63.7\right.$, confirmed as $\mathrm{CH}_{2}$ by DEPT), $-\mathrm{CH}\left(\mathrm{OCH}_{2} \boldsymbol{C H}_{3}\right) \mathrm{CH}_{2}-\left({ }^{1} \mathrm{H} \delta 1.20, \mathrm{t} ;{ }^{13} \mathrm{C} \delta 15.6\right.$, confirmed as $\mathrm{CH}_{3}$ by DEPT). 
Confirmation of $\mathrm{CH}_{3} \mathrm{CH}(\mathrm{OEt}) \mathrm{CH}_{2^{-}}$: In the COSY spectrum (Figure S-5f), the multiplet at $\delta$ 3.40 is correlated to a doublet at $\delta 1.15$ and a multiplet at $\delta 1.55$. The doublet at $\delta 1.15$ is correlated to a ${ }^{13} \mathrm{C} \mathrm{CH}_{3}$ signal at $\delta 19.8$ (confirmed as $\mathrm{CH}_{3}$ by DEPT) in the HMQC spectrum (Figure S-5k). The multiplet at $\delta 1.55$ is correlated to a ${ }^{13} \mathrm{C} \mathrm{CH}_{2}$ signal at $\delta 36.7$ (confirmed as $\mathrm{CH}_{2}$ by DEPT) in the HMQC spectrum (Figure $\mathrm{S}-5 \mathrm{k}$ ). These results and model compound data in Chart 1 confirm the assignment of the $\mathrm{CH}_{3} \mathrm{CH}(\mathrm{OEt}) \mathrm{CH}_{2}$ - unit.

Confirmation of $-\mathrm{CH}_{2} \mathrm{CH}(\mathrm{OEt}) \mathrm{CH}_{2}-$ : In the $\mathrm{COSY}$ spectrum (Figure S-5f), the multiplet at $\delta$ 3.23 is correlated to a multiplet at $\delta 1.50$. The multiplet at $\delta 1.50$ is correlated to a ${ }^{13} \mathrm{C} \mathrm{CH}_{2}$ signal at $\delta 34.2$ (confirmed as $\mathrm{CH}_{2}$ by DEPT) in the HMQC spectrum (Figure S-5k). These results and model compound data in Chart S-1 confirm the assignment of the $-\mathrm{CH}_{2} \mathrm{CH}(\mathrm{OEt}) \mathrm{CH}_{2}$ - unit. HMBC data (Figures S-51-n) in conjunction with model compound data showed $\boldsymbol{C H}_{2} \mathrm{CH}_{2} \mathrm{CH}(\mathrm{OEt}) \mathrm{CH}_{2} \boldsymbol{C H}_{2} \mathrm{P}\left({ }^{1} \mathrm{H} \delta 3.23 ;{ }^{13} \mathrm{C} \delta 25.4\right),-\mathrm{CH}_{2} \mathrm{C} \boldsymbol{H}\left(\mathrm{OCH}_{2} \mathrm{CH}_{3}\right) \mathrm{CH}_{2}-\left({ }^{1} \mathrm{H} \delta 3.23 ;{ }^{13} \mathrm{C} \delta\right.$ 63.7) and $-\mathrm{CH}_{2} \mathrm{CH}\left(\mathrm{OCH}_{2} \mathrm{CH}_{3}\right) \mathrm{CH}_{2}-\left({ }^{1} \mathrm{H} \delta 3.50 ;{ }^{13} \mathrm{C} \delta\right.$ 79.1) correlations, which confirm that the comonomer units are covalently linked to the polyethylene chain.

These assignments were further confirmed by comparison to the ethylene $/ \mathrm{CH}_{2}=\mathrm{CHBr}$ copolymer derived from the ethylene $/ \mathrm{CH}_{2}=\mathrm{CHOEt}$ copolymer (vide infra).

Key NMR Data for Ethylene/ $\mathbf{C H}_{2}=\mathbf{C H O E t}$ Copolymer. ${ }^{1} \mathbf{H}$ NMR $\left(\mathrm{CDCl}_{2} \mathrm{CDCl}_{2}, 70{ }^{\circ} \mathrm{C}\right.$, NMR500-2, SL319-11): $\delta 5.87$ (m, $\left.\mathrm{CH}_{2}=\mathrm{CHCH}_{2}-\right), 5.47$ (m, $\mathrm{CH}_{3} \mathrm{CH}=\mathrm{CHCH}_{2}$ ), 5.04 (d, $J=17$, $\left.\mathrm{CHH}^{\prime}=\mathrm{CH}-\right), 4.98 \quad\left(\mathrm{~d}, J=10, \mathrm{CH} H^{\prime}=\mathrm{CH}-\right), 3.50 \quad\left(\mathrm{q}, J=7, \mathrm{OCH}_{2} \mathrm{CH}_{3}\right), 3.40 \quad(\mathrm{~m}$, $\left.\mathrm{CH}_{3} \mathrm{CH}(\mathrm{OEt}) \mathrm{CH}_{2}-\right), 3.23$ (quintet, $J=6,-\mathrm{CH}_{2} \mathrm{CH}(\mathrm{OEt}) \mathrm{CH}_{2^{-}}$), 2.09 (m, $\mathrm{CH}_{2}=\mathrm{CHCH}_{2^{-}}$), 2.02 ($\left.\mathrm{CH}=\mathrm{CHCH}_{2}-\right), 1.68\left(\mathrm{~d}, J=6\right.$, trans- $\left.\mathrm{CH}_{3} \mathrm{CH}=\mathrm{CH}-\right), 1.65\left(\mathrm{~d}, J=6\right.$, cis- $\left.\mathrm{CH}_{3} \mathrm{CH}=\mathrm{CH}-\right), 1.55(\mathrm{~m}$, $\left.\mathrm{CH}_{3} \mathrm{CH}(\mathrm{OEt}) \mathrm{CH}_{2^{-}}\right), 1.50\left(\mathrm{~m},-\mathrm{CH}_{2} \mathrm{CH}(\mathrm{OEt}) \mathrm{CH}_{2^{-}}\right), 1.20\left(\mathrm{t}, J=7, \mathrm{OCH}_{2} \mathrm{CH}_{3}\right), 1.15$ (d, $J=6$, $\left.\mathrm{CH}_{3} \mathrm{CH}(\mathrm{OEt}) \mathrm{CH}_{2}-\right), 0.94$ (t, $\left.J=7, \mathrm{CH}_{3} \mathrm{CH}_{2}-\right), 0.90$ (d, $\left.J=6,-\mathrm{CH}\left(\mathrm{CH}_{3}\right)-\right) .{ }^{1} \mathrm{H}^{-}{ }^{1} \mathrm{H} \mathbf{C O S Y}$ correlations $\left(\mathrm{CDCl}_{2} \mathrm{CDCl}_{2}, \quad 70{ }^{\circ} \mathrm{C}, \quad \mathrm{NMR} 500-2, \quad \mathrm{SL} 319-21\right): \quad \delta / \delta \quad 3.50 \quad\left(\mathrm{OCH}_{2} \mathrm{CH}_{3}\right) / 1.20$ $\left(\mathrm{OCH}_{2} \mathrm{CH}_{3}\right), 3.40\left(\mathrm{CH}_{3} \mathrm{CH}(\mathrm{OEt}) \mathrm{CH}_{2^{-}}\right) / 1.55\left(\mathrm{CH}_{3} \mathrm{CH}(\mathrm{OEt}) \mathrm{CH}_{2^{-}}\right), 3.40\left(\mathrm{CH}_{3} \mathrm{CH}(\mathrm{OEt}) \mathrm{CH}_{2^{-}}\right) / 1.15$ $\left.\left(\mathrm{CH}_{3} \mathrm{CH}(\mathrm{OEt}) \mathrm{CH}_{2}-\right), 3.23 \quad\left(-\mathrm{CH}_{2} \mathrm{CH}(\mathrm{OEt}) \mathrm{CH}_{2}-\right) / 1.50 \quad\left(-\mathrm{CH}_{2} \mathrm{CH}(\mathrm{OEt}) \mathrm{CH}_{2}-\right) .{ }^{13} \mathbf{C}_{\{}{ }^{1} \mathbf{H}\right\} \quad$ NMR $\left(\mathrm{CDCl}_{2} \mathrm{CDCl}_{2}, \quad 70 \quad{ }^{\circ} \mathrm{C}, \quad\right.$ NMR500-2, $\quad$ SL319-13): $\delta \quad 79.1 \quad\left(-\mathrm{CH}_{2} \mathrm{CH}(\mathrm{OEt}) \mathrm{CH}_{2}-\right), \quad 74.8$ 
$\left(\mathrm{CH}_{3} \mathrm{CH}(\mathrm{OEt}) \mathrm{CH}_{2}-\right), 63.7\left(\mathrm{OCH}_{2} \mathrm{CH}_{3}\right), 63.0\left(\mathrm{OCH}_{2} \mathrm{CH}_{3}\right), 36.7\left(\mathrm{CH}_{3} \mathrm{CH}(\mathrm{OEt}) \mathrm{CH}_{2}-\right), 34.2$ ($\left.\mathrm{CH}_{2} \mathrm{CH}(\mathrm{OEt}) \mathrm{CH}_{2}-\right), \quad 29.7 \quad\left(-\mathrm{CH}_{2} \mathrm{CH}_{2} \mathrm{CH}_{2} \mathrm{CH}(\mathrm{OEt}) \mathrm{CH}_{2} \mathrm{CH}_{2} \mathrm{CH}_{2}-\right), \quad 29.5 \quad\left(-\mathrm{CH}_{2}-\right), \quad 25.42$ $\left(\mathrm{CH}_{3} \mathrm{CH}(\mathrm{OEt}) \mathrm{CH}_{2} \mathrm{CH}_{2}-\right), 25.36\left(-\mathrm{CH}_{2} \mathrm{CH}_{2} \mathrm{CH}(\mathrm{OEt}) \mathrm{CH}_{2} \mathrm{CH}_{2}-\right), 19.8\left(\mathrm{CH}_{3} \mathrm{CH}(\mathrm{OEt}) \mathrm{CH}_{2}-\right), 15.6$ $\left(\mathrm{OCH}_{2} \mathrm{CH}_{3}\right)$; assignments confirmed by DEPT. HMQC $\left(\mathrm{CDCl}_{2} \mathrm{CDCl}_{2}, 70{ }^{\circ} \mathrm{C}\right.$, NMR500-2, SL319-HMQC.5): $\quad \delta / \delta \quad 79.1 \quad\left(-\mathrm{CH}_{2} \mathrm{CH}(\mathrm{OEt}) \mathrm{CH}_{2}-\right) / 3.23 \quad\left(-\mathrm{CH}_{2} \mathrm{CH}(\mathrm{OEt}) \mathrm{CH}_{2}-\right), \quad 74.8$ $\left(\mathrm{CH}_{3} \mathrm{CH}(\mathrm{OEt}) \mathrm{CH}_{2}-\right) / 3.40 \quad\left(\mathrm{CH}_{3} \mathrm{CH}(\mathrm{OEt}) \mathrm{CH}_{2}-\right), \quad 63.7 \quad\left(\mathrm{OCH}_{2} \mathrm{CH}_{3}\right) / 3.50 \quad\left(\mathrm{OCH}_{2} \mathrm{CH}_{3}\right), \quad 36.7$ $\left(\mathrm{CH}_{3} \mathrm{CH}(\mathrm{OEt}) \mathrm{CH}_{2}-\right) / 1.55 \quad\left(\mathrm{CH}_{3} \mathrm{CH}(\mathrm{OEt}) \mathrm{CH}_{2^{-}}\right), \quad 34.2 \quad\left(-\mathrm{CH}_{2} \mathrm{CH}(\mathrm{OEt}) \mathrm{CH}_{2^{-}}\right) / 1.50$ $\left.\mathrm{CH}_{2} \mathrm{CH}(\mathrm{OEt}) \mathrm{CH}_{2}-\right), \quad 25.4 \quad\left(-\mathrm{CH}_{2} \mathrm{CH}_{2} \mathrm{CH}\left(\mathrm{OCH}_{2} \mathrm{CH}_{3}\right) \mathrm{CH}_{2} \mathrm{CH}_{2}-\right) / 1.40$ $\left.\mathrm{CH}_{2} \mathrm{CH}_{2} \mathrm{CH}\left(\mathrm{OCH}_{2} \mathrm{CH}_{3}\right) \mathrm{CH}_{2} \mathrm{CH}_{2}-\right), 19.8\left(\mathrm{CH}_{3} \mathrm{CH}(\mathrm{OEt}) \mathrm{CH}_{2}-\right) / 1.15 \quad\left(\mathrm{CH}_{3} \mathrm{CH}(\mathrm{OEt}) \mathrm{CH}_{2}-\right), 15.6$ $\left(\mathrm{OCH}_{2} \mathrm{CH}_{3}\right) / 1.20\left(\mathrm{OCH}_{2} \mathrm{CH}_{3}\right) . \mathbf{H M B C}\left(\mathrm{CDCl}_{2} \mathrm{CDCl}_{2}, 70{ }^{\circ} \mathrm{C}, \mathrm{NMR} 500-2, \mathrm{SL} 319-18\right): \delta / \delta 3.50$ $\left(\mathrm{OCH}_{2} \mathrm{CH}_{3}\right) / 79.1 \quad\left(-\mathrm{CH}_{2} \mathrm{CH}(\mathrm{OEt}) \mathrm{CH}_{2^{-}}\right), 3.23\left(-\mathrm{CH}_{2} \mathrm{CH}(\mathrm{OEt}) \mathrm{CH}_{2^{-}}\right) / 63.7 \quad\left(\mathrm{OCH}_{2} \mathrm{CH}_{3}\right), 3.23$ $\left.\mathrm{CH}_{2} \mathrm{CH}(\mathrm{OEt}) \mathrm{CH}_{2}-\right) / 25.4\left(-\mathrm{CH}_{2} \mathrm{CH}_{2} \mathrm{CH}(\mathrm{OEt}) \mathrm{CH}_{2} \mathrm{CH}_{2}-\right)$.

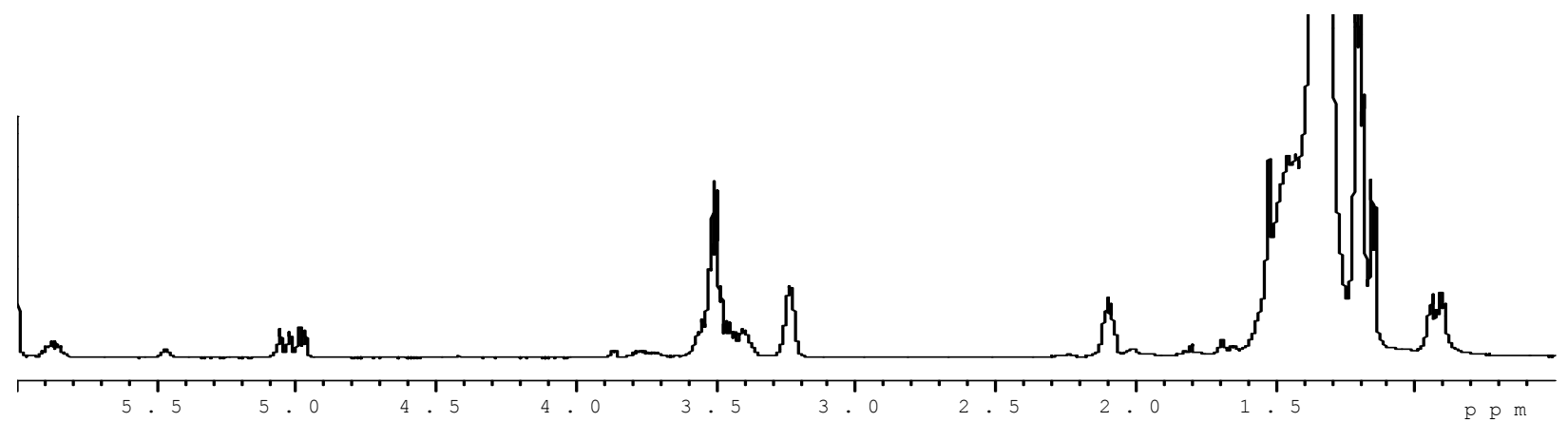

Figure S-5a. ${ }^{1} \mathrm{H}$ NMR $\left(\mathrm{CDCl}_{2} \mathrm{CDCl}_{2}, 70{ }^{\circ} \mathrm{C}\right)$ of ethylene $/ \mathrm{CH}_{2}=\mathrm{CHOEt}$ copolymer: vertical expansion. The signal at $\delta 6.00$ is from the solvent.

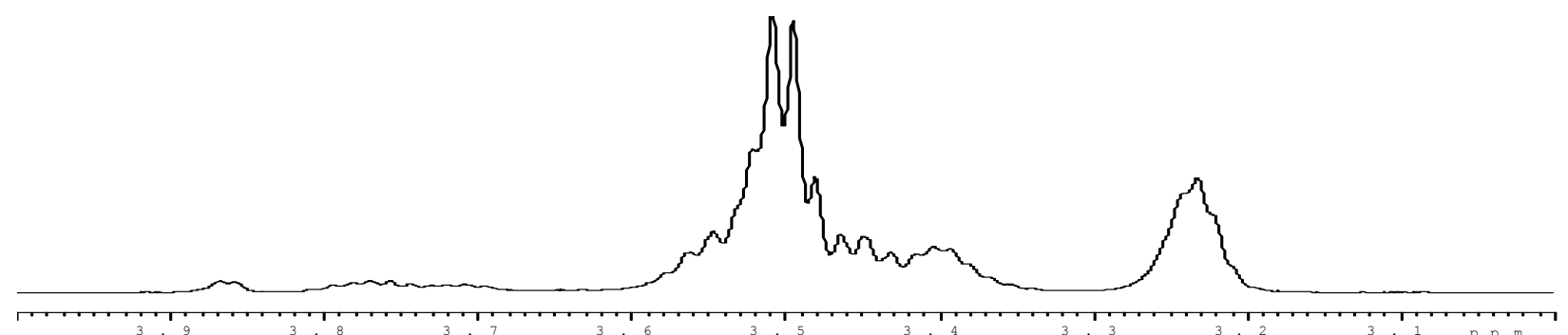


Figure S-5b. ${ }^{1} \mathrm{H}$ NMR $\left(\mathrm{CDCl}_{2} \mathrm{CDCl}_{2}, 70{ }^{\circ} \mathrm{C}\right)$ of ethylene $/ \mathrm{CH}_{2}=\mathrm{CHOEt}$ copolymer: expansion of the $\delta$ 4.0-3.0 region.

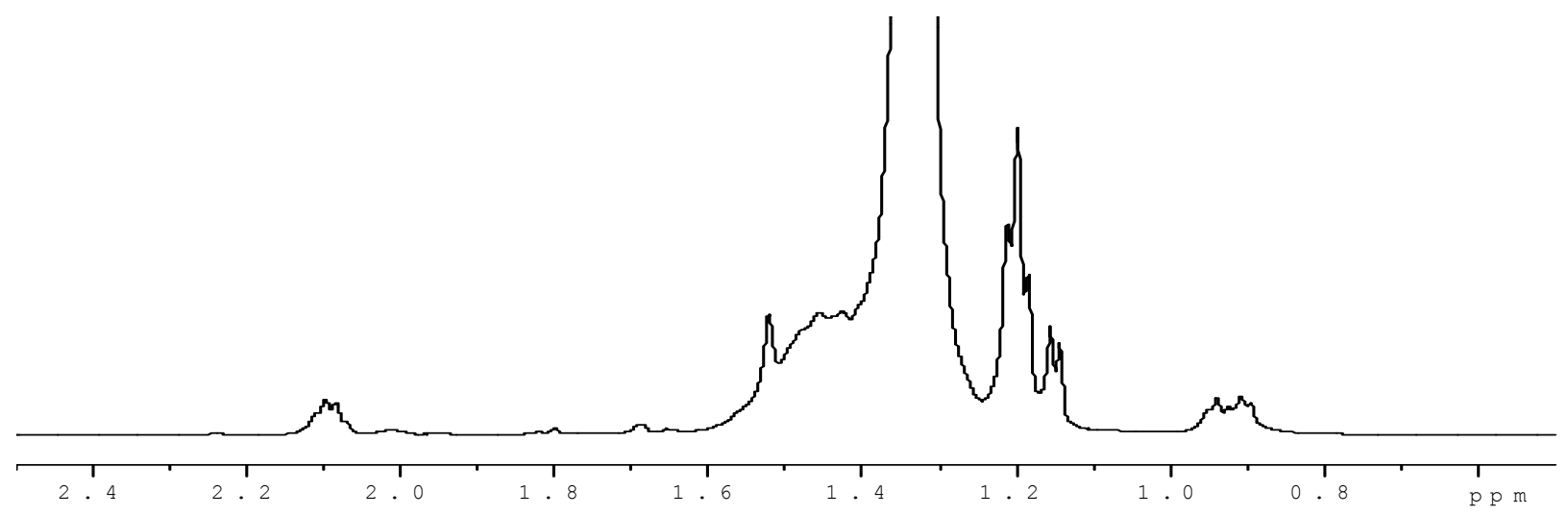

Figure S-5c. ${ }^{1} \mathrm{H}$ NMR $\left(\mathrm{CDCl}_{2} \mathrm{CDCl}_{2}, 70{ }^{\circ} \mathrm{C}\right)$ of ethylene/ $\mathrm{CH}_{2}=\mathrm{CHOEt}$ copolymer: expansion of the $\delta$ 2.5-0.5 region.

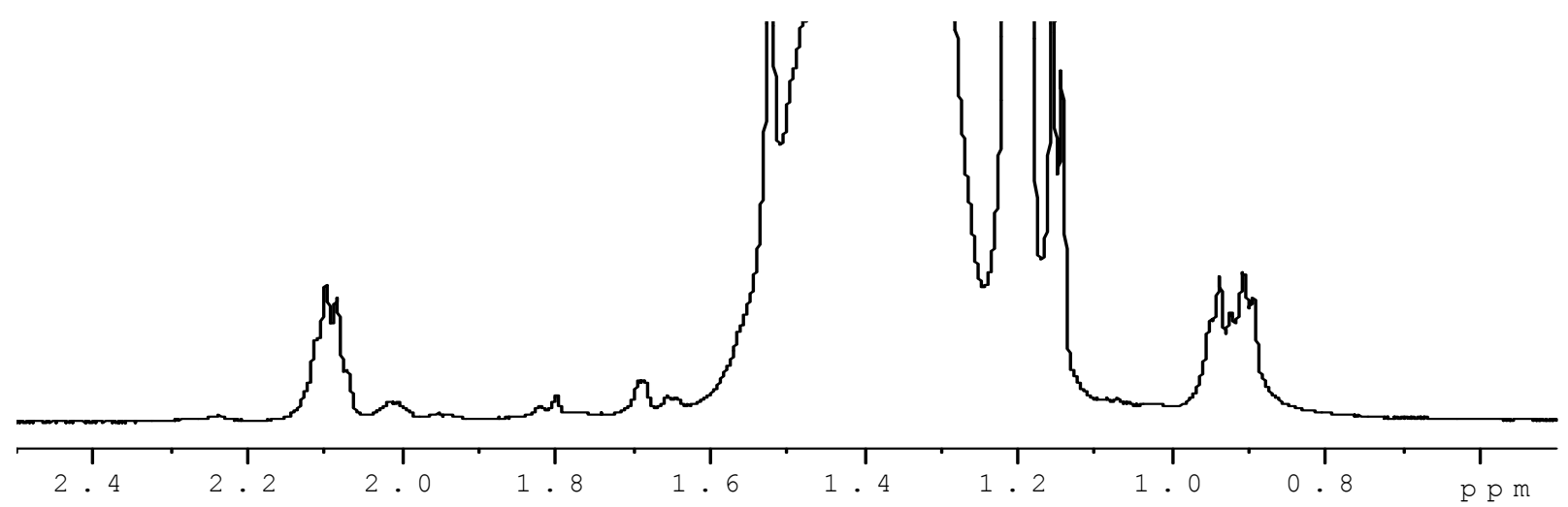

Figure S-5d. ${ }^{1} \mathrm{H} \mathrm{NMR}\left(\mathrm{CDCl}_{2} \mathrm{CDCl}_{2}, 70{ }^{\circ} \mathrm{C}\right)$ of ethylene $/ \mathrm{CH}_{2}=\mathrm{CHOEt}$ copolymer: expansion of the $\delta$ 2.5-0.5 region. 


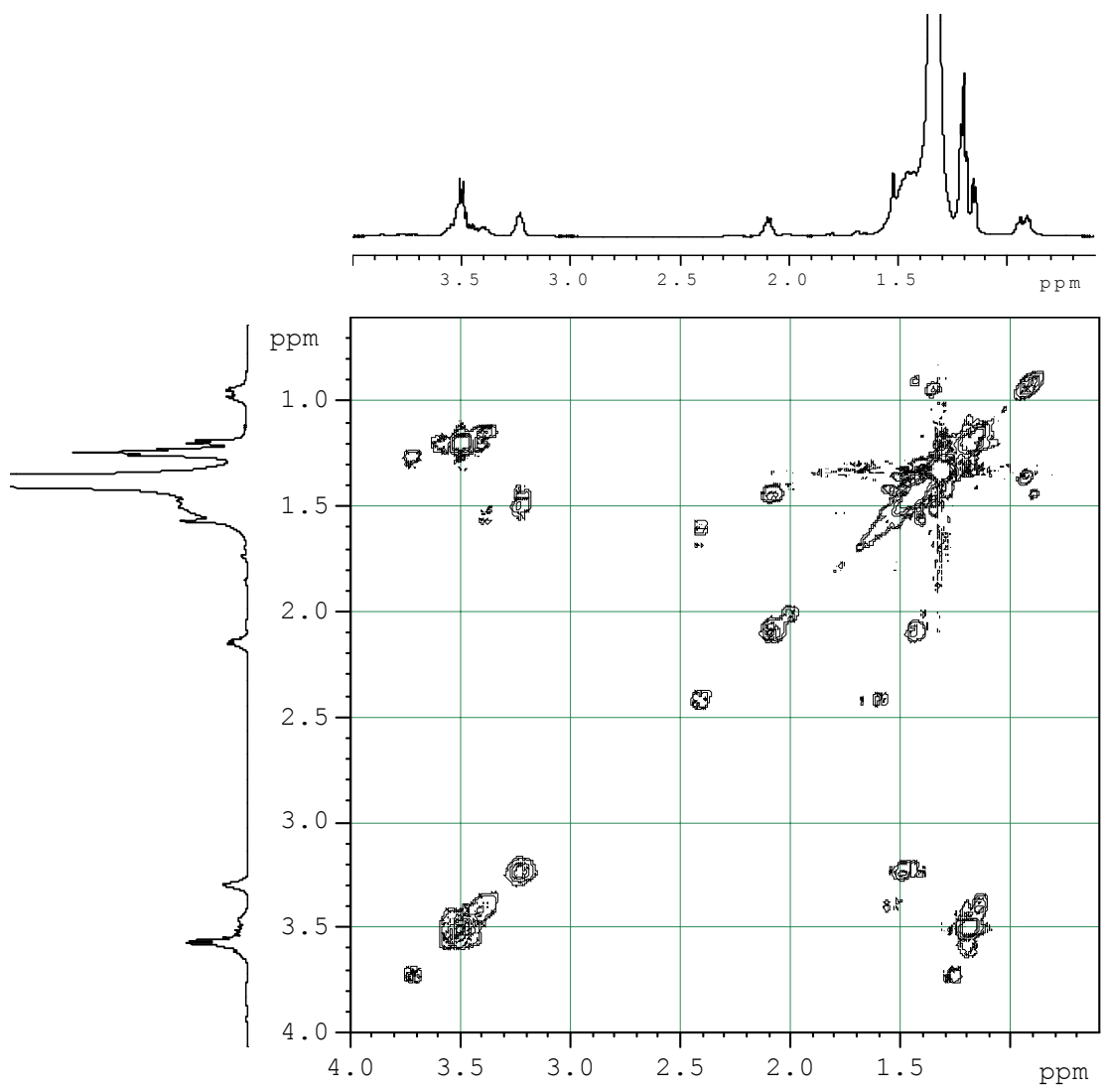

Figure S-5e. $\mathrm{COSY} \mathrm{NMR}\left(\mathrm{CDCl}_{2} \mathrm{CDCl}_{2}, 70{ }^{\circ} \mathrm{C}\right)$ of ethylene $/ \mathrm{CH}_{2}=\mathrm{CHOEt}$ copolymer: expansion of the $\delta 4.0-0.5 ; 4.0-0.5$ region. 


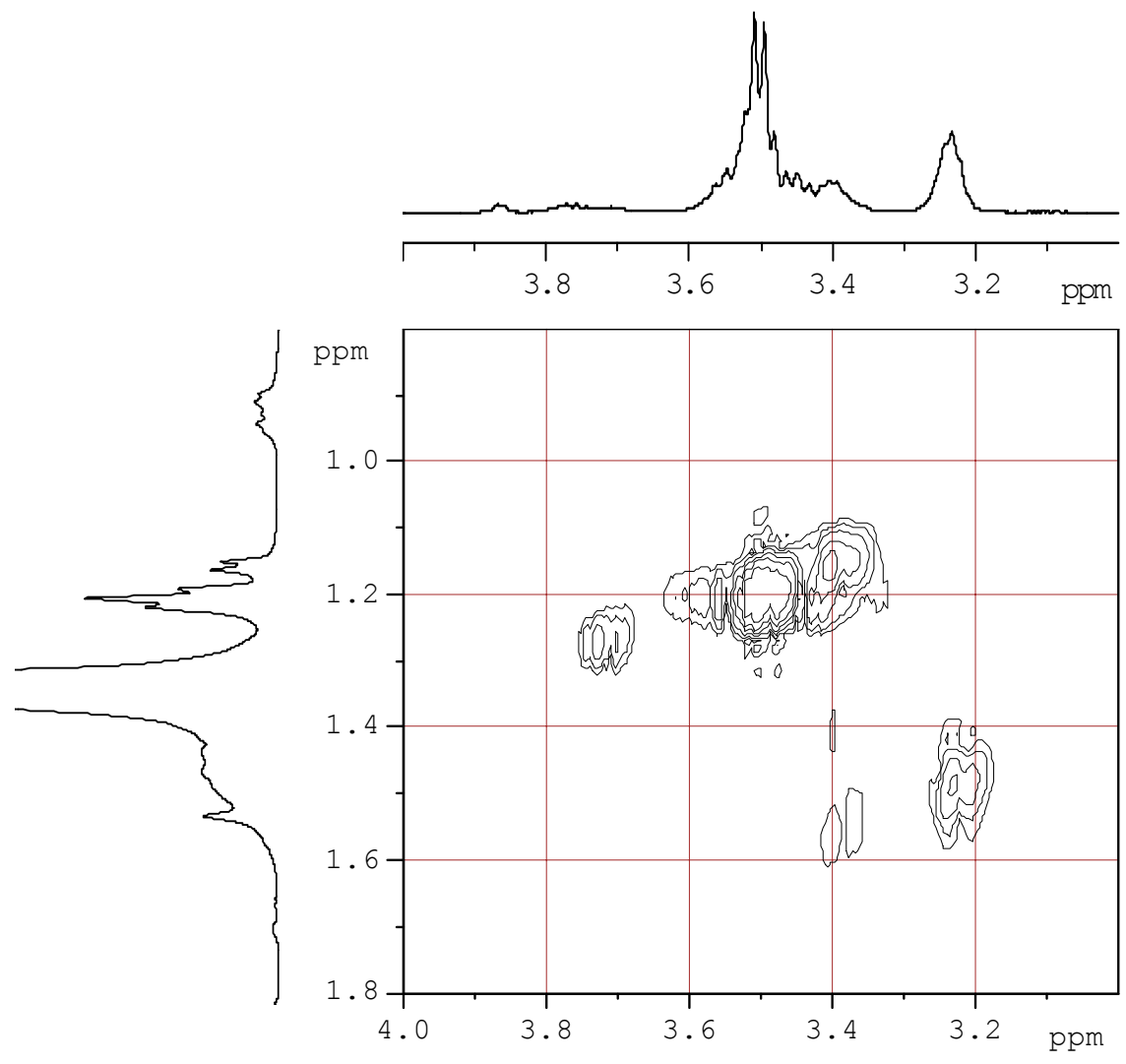

Figure S-5f. COSY NMR $\left(\mathrm{CDCl}_{2} \mathrm{CDCl}_{2}, 70{ }^{\circ} \mathrm{C}\right)$ of ethylene $/ \mathrm{CH}_{2}=\mathrm{CHOEt}$ copolymer: expansion of the $\delta 4.0-3.0 ; 1.8-0.8$ region.

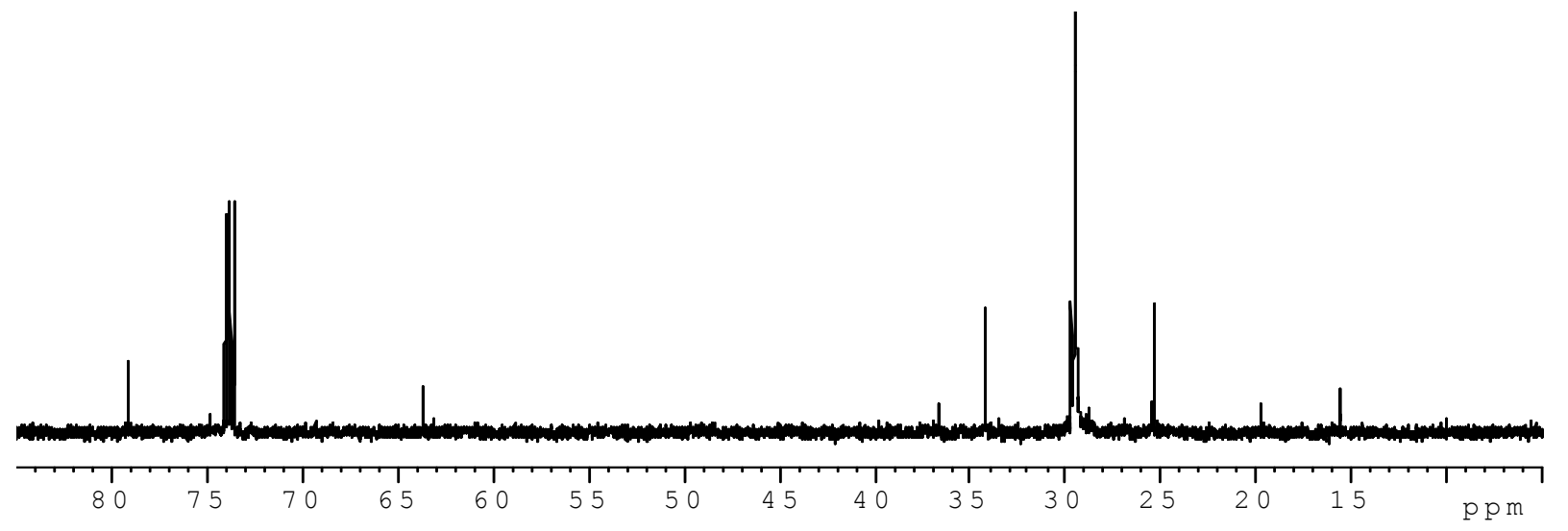

Figure S-5g. ${ }^{13} \mathrm{C}$ NMR $\left(\mathrm{CDCl}_{2} \mathrm{CDCl}_{2}, 70{ }^{\circ} \mathrm{C}\right)$ of ethylene $/ \mathrm{CH}_{2}=\mathrm{CHOEt}$ copolymer: expansion of the $\delta 85-5$ region. The signal at $\delta 73.8$ is from the solvent. 


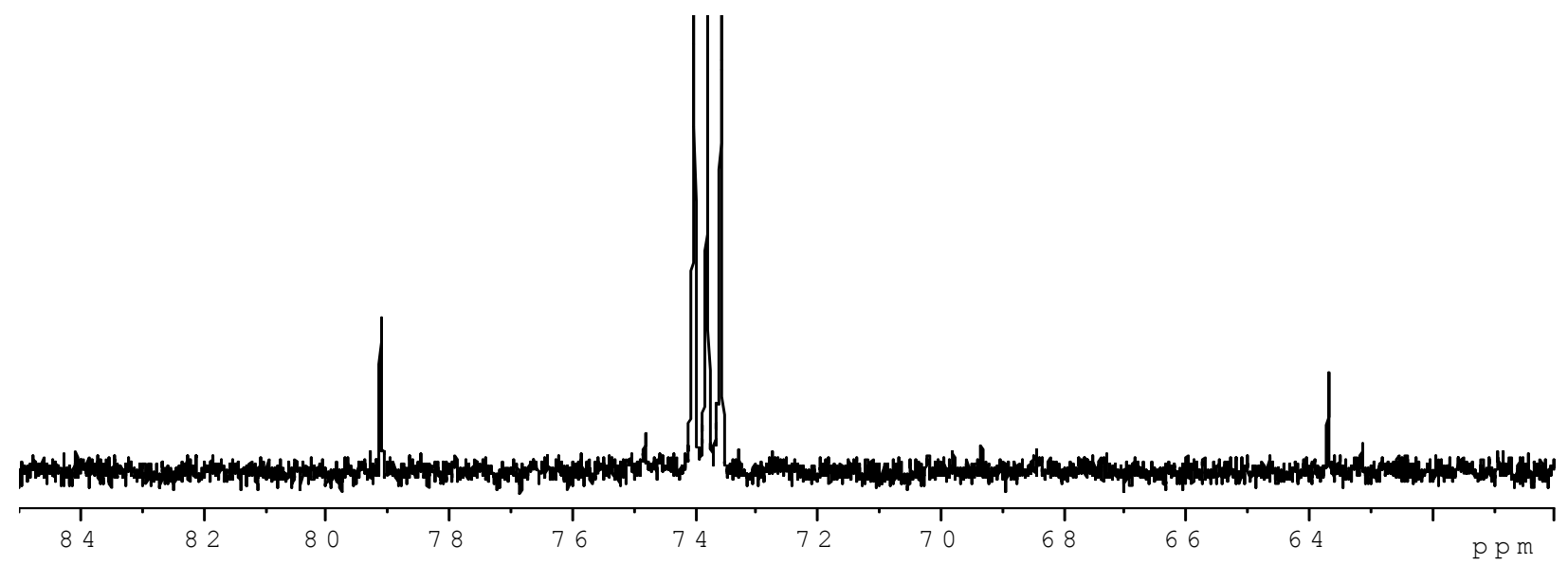

Figure S-5h. ${ }^{13} \mathrm{C}$ NMR $\left(\mathrm{CDCl}_{2} \mathrm{CDCl}_{2}, 70{ }^{\circ} \mathrm{C}\right)$ of ethylene/ $\mathrm{CH}_{2}=\mathrm{CHOEt}$ copolymer: expansion of the $\delta 85-60$ region. The signal at $\delta 73.8$ is from the solvent.

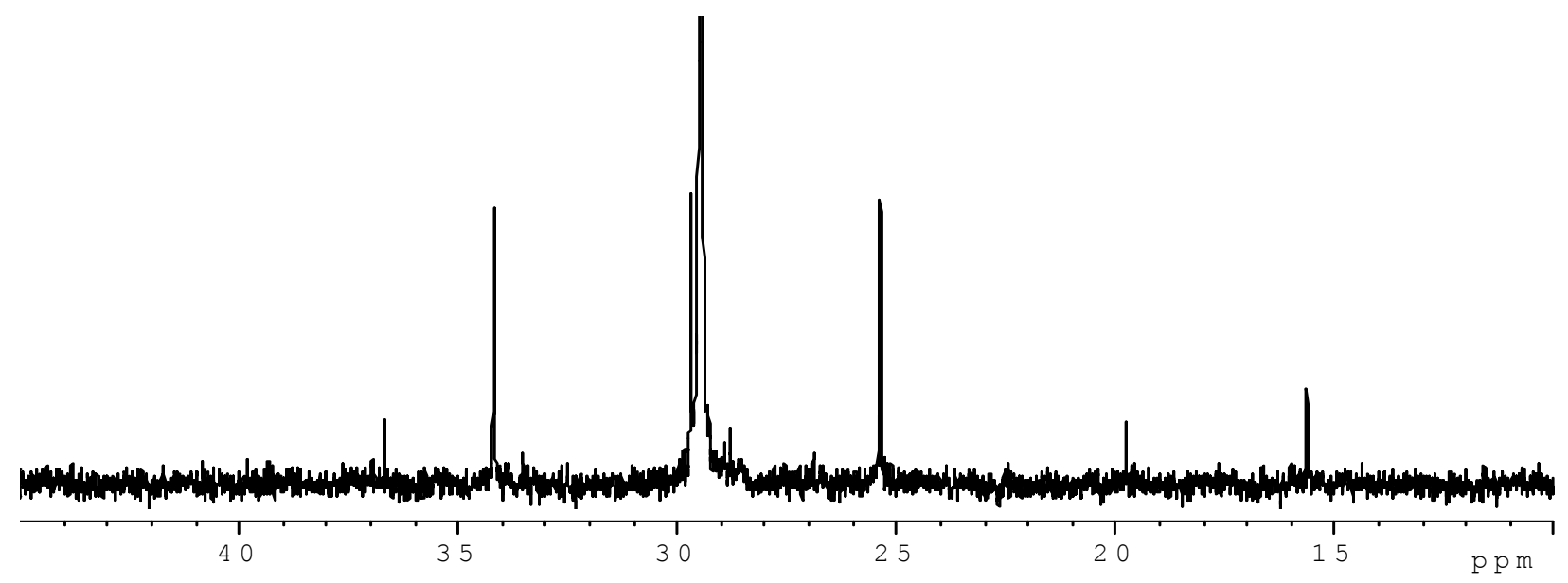

Figure S-5i. ${ }^{13} \mathrm{C}$ NMR $\left(\mathrm{CDCl}_{2} \mathrm{CDCl}_{2}, 70{ }^{\circ} \mathrm{C}\right)$ of ethylene $/ \mathrm{CH}_{2}=\mathrm{CHOEt}$ copolymer: expansion of the $\delta 45-10$ region. 


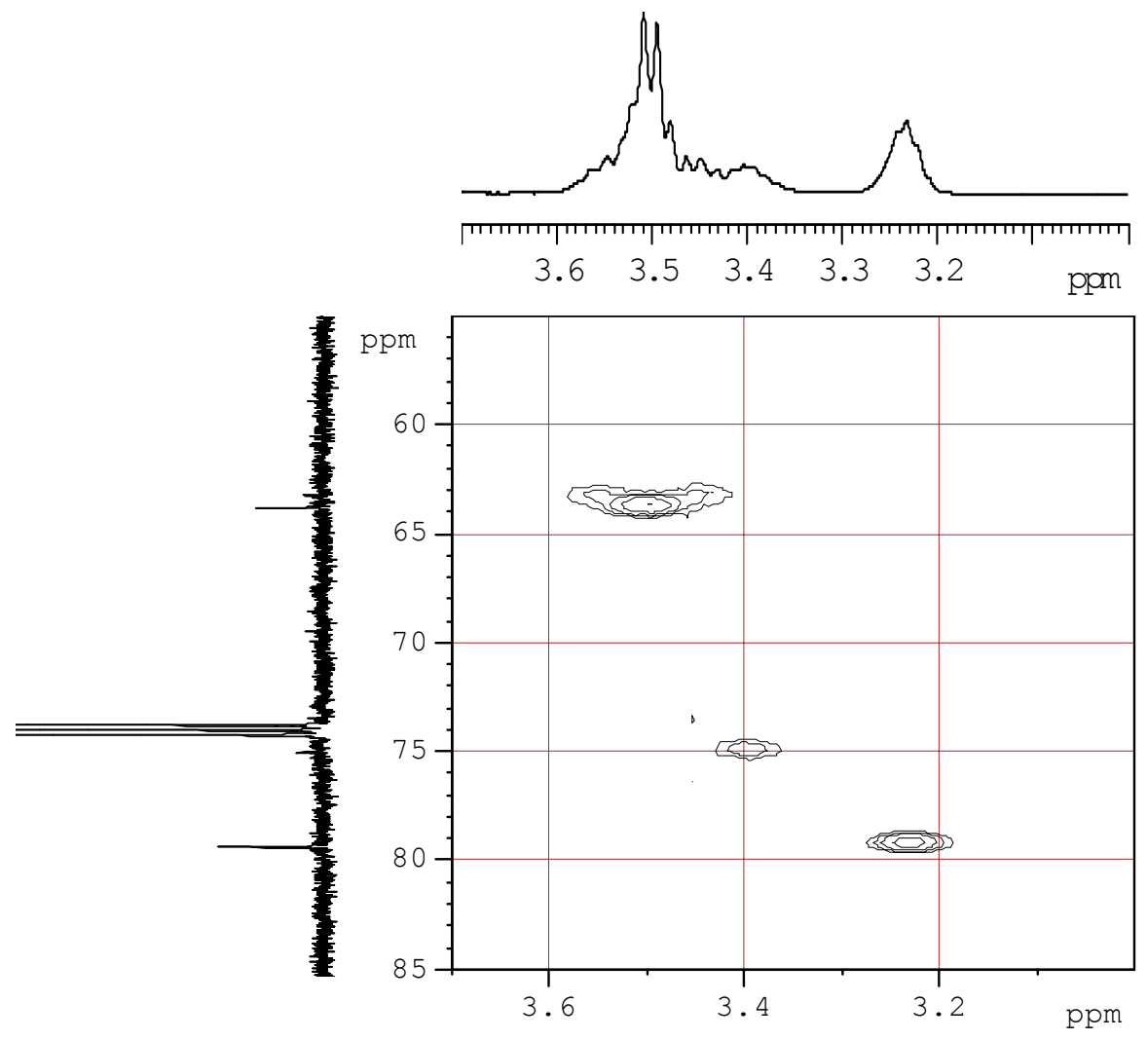

Figure S-5j. HMQC NMR $\left(\mathrm{CDCl}_{2} \mathrm{CDCl}_{2}, 70{ }^{\circ} \mathrm{C}\right)$ of ethylene $/ \mathrm{CH}_{2}=\mathrm{CHOEt}$ copolymer: expansion of the $\delta 3.7-3.0 ; 85-55$ region. 


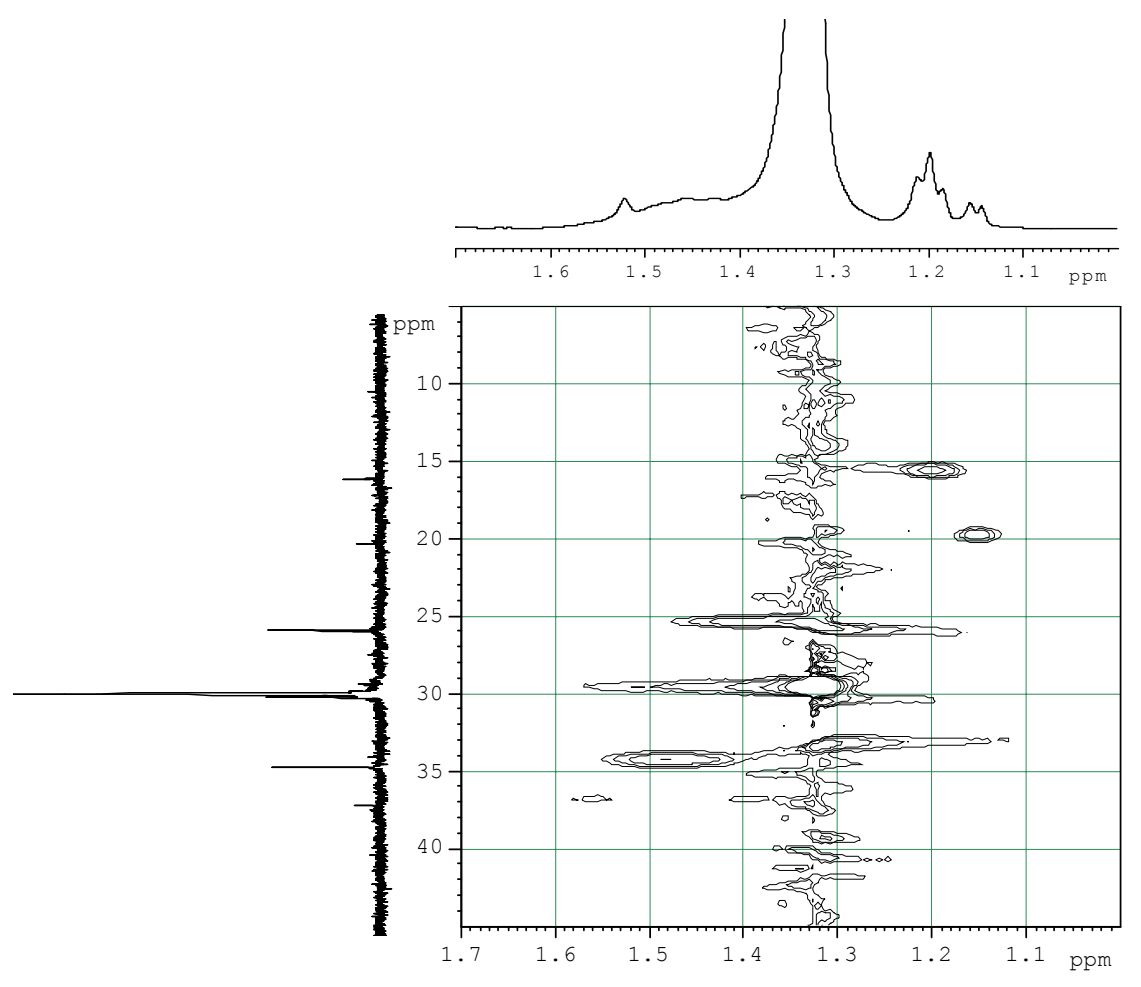

Figure S-5k. HMQC NMR $\left(\mathrm{CDCl}_{2} \mathrm{CDCl}_{2}, 70{ }^{\circ} \mathrm{C}\right)$ of ethylene $/ \mathrm{CH}_{2}=\mathrm{CHOEt}$ copolymer: expansion of the $\delta 1.7-1.0 ; 45-5$ region.

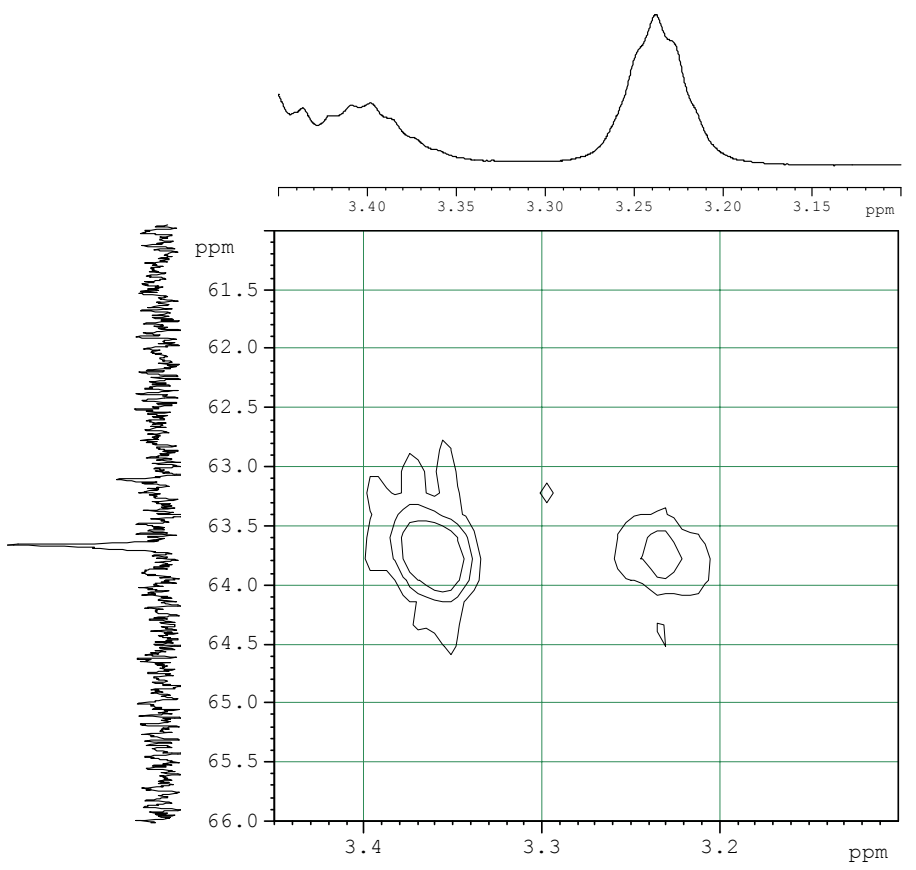

Figure S-5l. $\mathrm{HMBC} \mathrm{NMR}\left(\mathrm{CDCl}_{2} \mathrm{CDCl}_{2}, 70{ }^{\circ} \mathrm{C}\right)$ of ethylene $/ \mathrm{CH}_{2}=\mathrm{CHOEt}$ copolymer: expansion of the $\delta 3.45-3.1 ; 66-61$ region. 

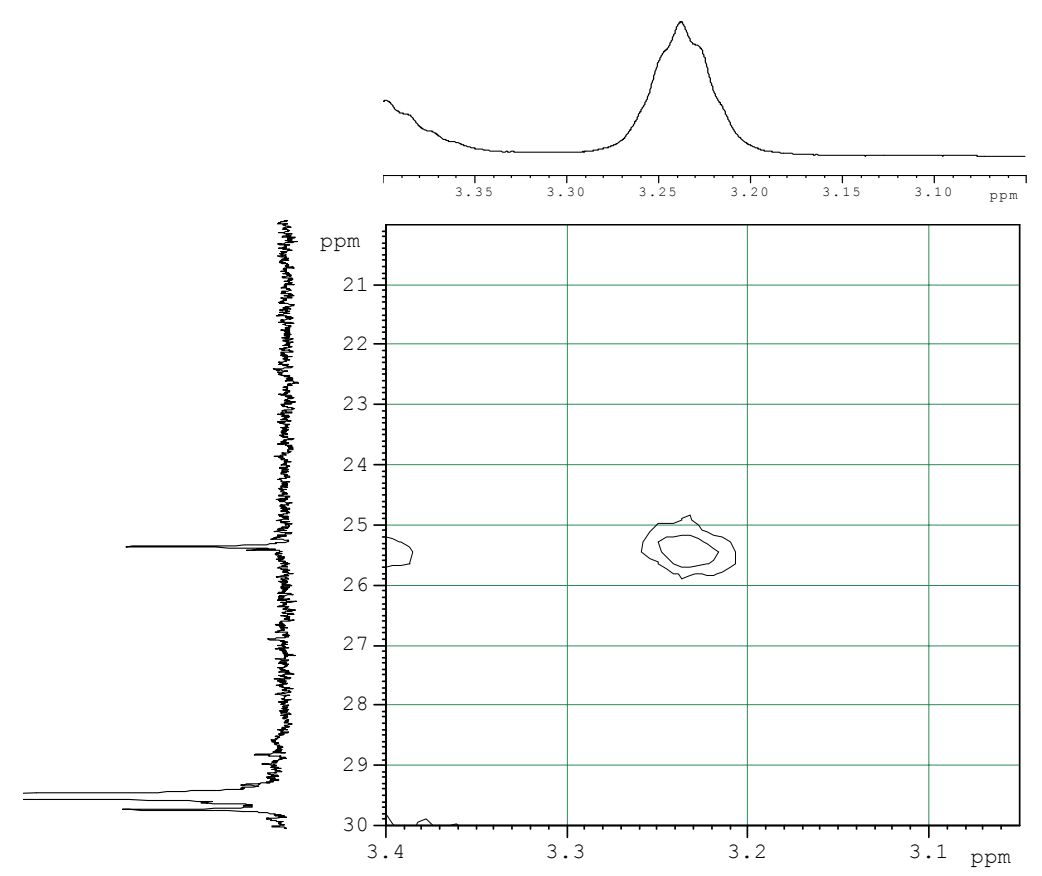

Figure S-5m. HMBC NMR $\left(\mathrm{CDCl}_{2} \mathrm{CDCl}_{2}, 70{ }^{\circ} \mathrm{C}\right)$ of ethylene $/ \mathrm{CH}_{2}=\mathrm{CHOEt}$ copolymer: expansion of the $\delta 3.4-3.05 ; 30-20$ region.

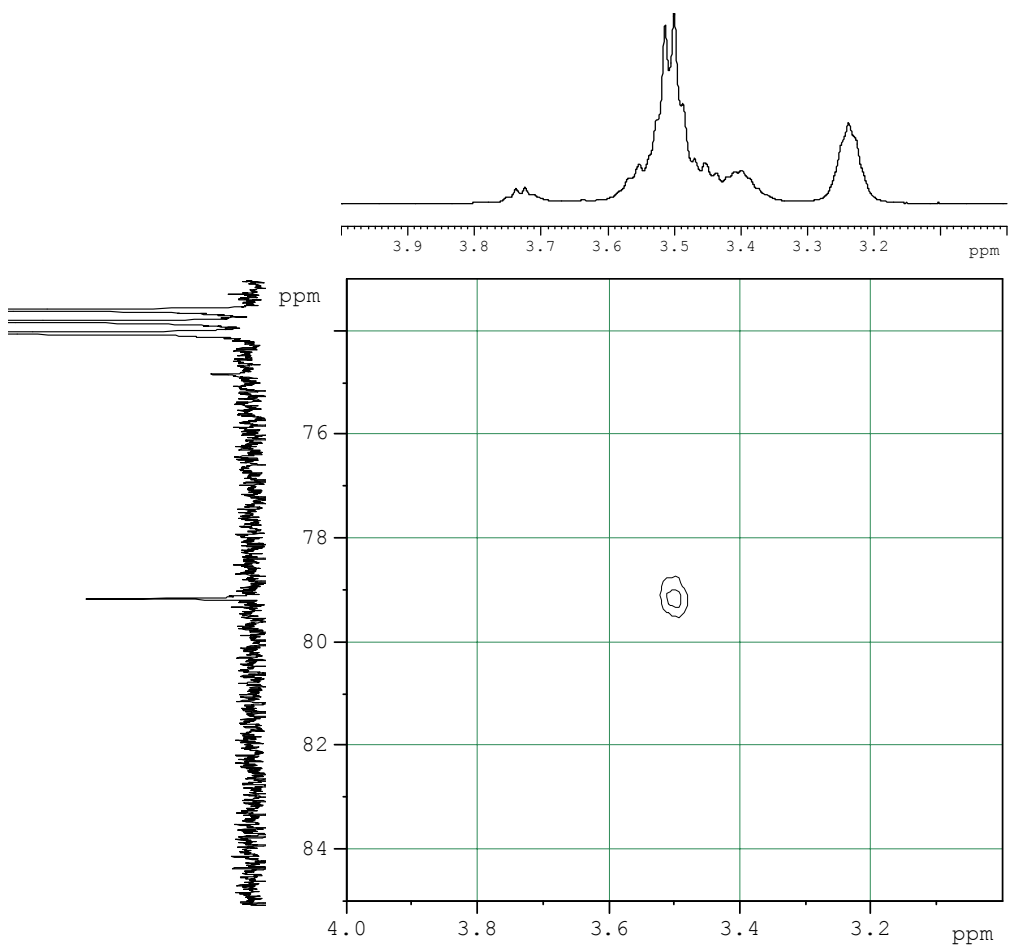

Figure S-5n. $\mathrm{HMBC} \mathrm{NMR}\left(\mathrm{CDCl}_{2} \mathrm{CDCl}_{2}, 70{ }^{\circ} \mathrm{C}\right)$ of ethylene $/ \mathrm{CH}_{2}=\mathrm{CHOEt}$ copolymer: expansion of the $\delta 4.0-3.0 ; 85-73$ region. 


\section{Conversion of Ethylene $/ \mathrm{CH}_{2}=\mathrm{CHOEt}$ Copolymer to Ethylene $/ \mathrm{CH}_{2}=\mathrm{CHBr}$ Copolymer}

by Reaction with HBr. (NB3-P52) An NMR tube was charged with ethylene $/ \mathrm{CH}_{2}=\mathrm{CHOEt}$ copolymer (40 mg, $\left.4.2 \mathrm{~mol} \% \mathrm{CH}_{2}=\mathrm{CHOEt}\right)$ and $\mathrm{CDCl}_{2} \mathrm{CDCl}_{2}(0.4 \mathrm{~mL})$. NMR analysis showed that the copolymer contained $\mathrm{CH}_{3} \mathrm{CH}(\mathrm{OEt}) \mathrm{CH}_{2}-(1.5 \mathrm{~mol} \%),-\mathrm{CH}_{2} \mathrm{CH}(\mathrm{OEt}) \mathrm{CH}_{2}-(2.7 \mathrm{~mol} \%)$, $\mathrm{CH}_{2} \mathrm{CH}=\mathrm{CH}_{2}(0.84 \mathrm{~mol} \%)$ and $-\mathrm{CH}_{2} \mathrm{CH}=\mathrm{CHMe}(0.14 \mathrm{~mol} \%)$. $\mathrm{HBr}(2.0 \mathrm{mmol})$ was added by vacuum transfer at $-196{ }^{\circ} \mathrm{C}$. The tube was warmed to room temperature, shaken and heated to 80 ${ }^{\circ} \mathrm{C}$ for 2 days. The volatiles were removed under vacuum at $60{ }^{\circ} \mathrm{C} . \mathrm{NMR}$ analysis of the product showed that the copolymer contained $\mathrm{CH}_{3} \mathrm{CH}(\mathrm{Br}) \mathrm{CH}_{2}-(1.5 \mathrm{~mol} \%$, see $\mathbf{S - 1 0}$, Chart S-1), $\mathrm{CH}_{2} \mathrm{CH}(\mathrm{Br}) \mathrm{CH}_{2-}(2.8 \mathrm{~mol} \%$, see S-8, S-9, Chart $\mathrm{S}-1), \mathrm{CH}_{3} \mathrm{CH}(\mathrm{OH}) \mathrm{CH}_{2^{-}}(0.13 \mathrm{~mol} \%)$, $\mathrm{CH}_{2} \mathrm{CH}(\mathrm{OH}) \mathrm{CH}_{2-}(0.23 \mathrm{~mol} \%),-\mathrm{CH}(\mathrm{OEt}) \mathrm{CH}_{2}-(0.62 \mathrm{~mol} \%)$ and $-\mathrm{CH}_{2} \mathrm{CH}=\mathrm{CHMe}(0.14 \mathrm{~mol} \%)$. Therefore, the $\mathrm{CH}_{3} \mathrm{CH}(\mathrm{OEt}) \mathrm{CH}_{2-}$ and $-\mathrm{CH}_{2} \mathrm{CH}(\mathrm{OEt}) \mathrm{CH}_{2^{-}}$groups were converted to $\mathrm{CH}_{3} \mathrm{CH}(\mathrm{Br}) \mathrm{CH}_{2}$ - and $-\mathrm{CH}_{2} \mathrm{CH}(\mathrm{Br}) \mathrm{CH}_{2}$ - groups. In addition, vinyl groups $\left(-\mathrm{CH}_{2} \mathrm{CH}=\mathrm{CH}_{2}\right)$ were converted to the $\mathrm{CH}_{3} \mathrm{CH}(\mathrm{Br}) \mathrm{CH}_{2}$ - groups and 2-olefins $\left(-\mathrm{CH}_{2} \mathrm{CH}=\mathrm{CHMe}\right)$, the latter could further react with $\mathrm{HBr}$ to generate the $-\mathrm{CH}_{2} \mathrm{CH}(\mathrm{Br}) \mathrm{CH}_{2}$ - groups.

Key Data for Ethylene $/ \mathbf{C H}_{2}=\mathbf{C H B r}$ Copolymer. ${ }^{1} \mathbf{H} \mathbf{N M R}\left(\mathrm{CDCl}_{2} \mathrm{CDCl}_{2}, 100{ }^{\circ} \mathrm{C}\right.$, NMR400-1, SL319-3.1): $\delta 4.21$ (sextet, $\left.J=7, \mathrm{CH}_{3} \mathrm{CH}(\mathrm{Br}) \mathrm{CH}_{2}-\right), 4.10\left(\mathrm{~m},-\mathrm{CH}_{2} \mathrm{CH}(\mathrm{Br}) \mathrm{CH}_{2^{-}}\right.$), $1.89\left(\mathrm{~m},-\mathrm{CH}(\mathrm{Br}) \mathrm{CH}_{2}-\right), 1.76\left(\mathrm{~d}, J=7, \mathrm{CH}_{3} \mathrm{CH}(\mathrm{Br}) \mathrm{CH}_{2}-\right) .{ }^{13} \mathbf{C}\left\{{ }^{1} \mathbf{H}\right\} \mathbf{N M R}\left(\mathrm{CDCl}_{2} \mathrm{CDCl}_{2}, 100{ }^{\circ} \mathrm{C}\right.$, NMR400-1, SL319-3.3): $\quad \delta \quad 58.4 \quad\left(-\mathrm{CH}_{2} \mathrm{CH}(\mathrm{Br}) \mathrm{CH}_{2^{-}}\right), \quad 51.3 \quad\left(\mathrm{CH}_{3} \mathrm{CH}(\mathrm{Br}) \mathrm{CH}_{2^{-}}\right), \quad 41.1$ $\left(\mathrm{CH}_{3} \mathrm{CH}(\mathrm{Br}) \mathrm{CH}_{2}-\right), \quad 39.0 \quad\left(-\mathrm{CH}_{2} \mathrm{CH}(\mathrm{Br}) \mathrm{CH}_{2^{-}}\right), \quad 27.4 \quad\left(\mathrm{CH}_{3} \mathrm{CH}\left(\mathrm{Br}^{-} \mathrm{CH}_{2} \mathrm{CH}_{2^{-}}\right), \quad 27.3 \quad\right.$ $\left.\mathrm{CH}_{2} \mathrm{CH}_{2} \mathrm{CH}(\mathrm{Br}) \mathrm{CH}_{2} \mathrm{CH}_{2-}\right), 26.0\left(\mathrm{CH}_{3} \mathrm{CH}(\mathrm{Br}) \mathrm{CH}_{2}-\right)$.

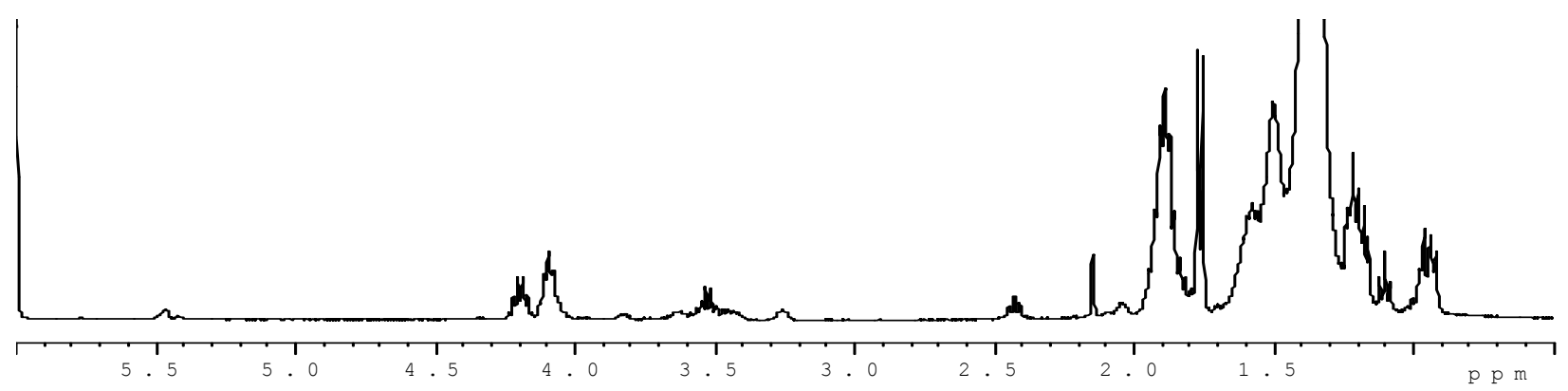

Figure S-6a. ${ }^{1} \mathrm{H} \mathrm{NMR}\left(\mathrm{CDCl}_{2} \mathrm{CDCl}_{2}, 100{ }^{\circ} \mathrm{C}\right)$ of ethylene $/ \mathrm{CH}_{2}=\mathrm{CHBr}$ copolymer: vertical expansion. The signal at $\delta 6.00$ is from the solvent. 


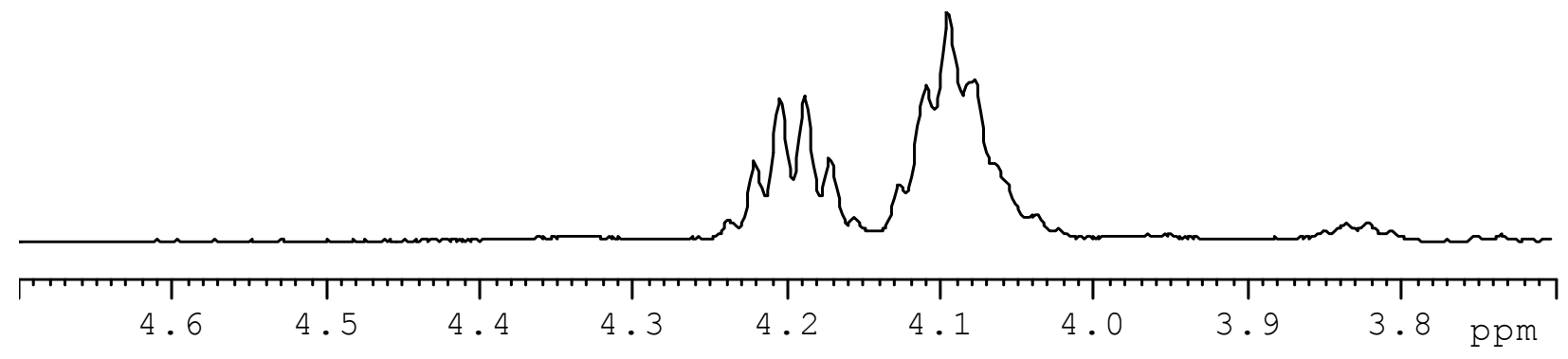

Figure S-6b. ${ }^{1} \mathrm{H}$ NMR $\left(\mathrm{CDCl}_{2} \mathrm{CDCl}_{2}, 100{ }^{\circ} \mathrm{C}\right)$ of ethylene $/ \mathrm{CH}_{2}=\mathrm{CHBr}$ copolymer: expansion of the $\delta$ 4.7-3.7 region. The signal at $\delta 3.83$ is from the $\mathrm{CH}_{3} \mathrm{CH}(\mathrm{OH}) \mathrm{CH}_{2}-$.

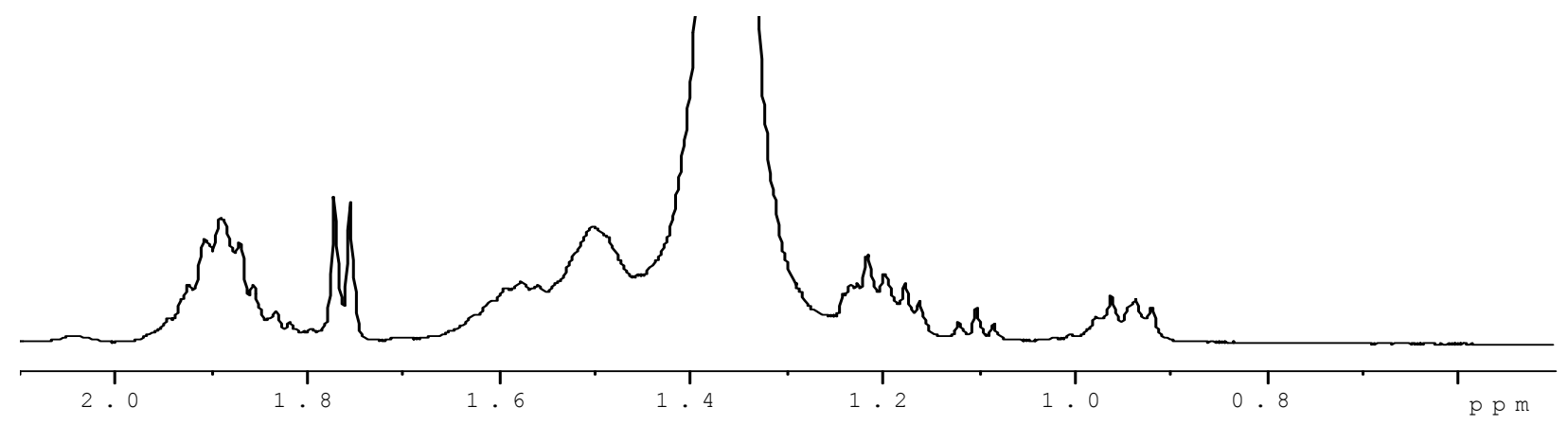

Figure S-6c. ${ }^{1} \mathrm{H}$ NMR $\left(\mathrm{CDCl}_{2} \mathrm{CDCl}_{2}, 100{ }^{\circ} \mathrm{C}\right)$ of ethylene $/ \mathrm{CH}_{2}=\mathrm{CHBr}$ copolymer: expansion of the $\delta$ 2.1-0.5 region.

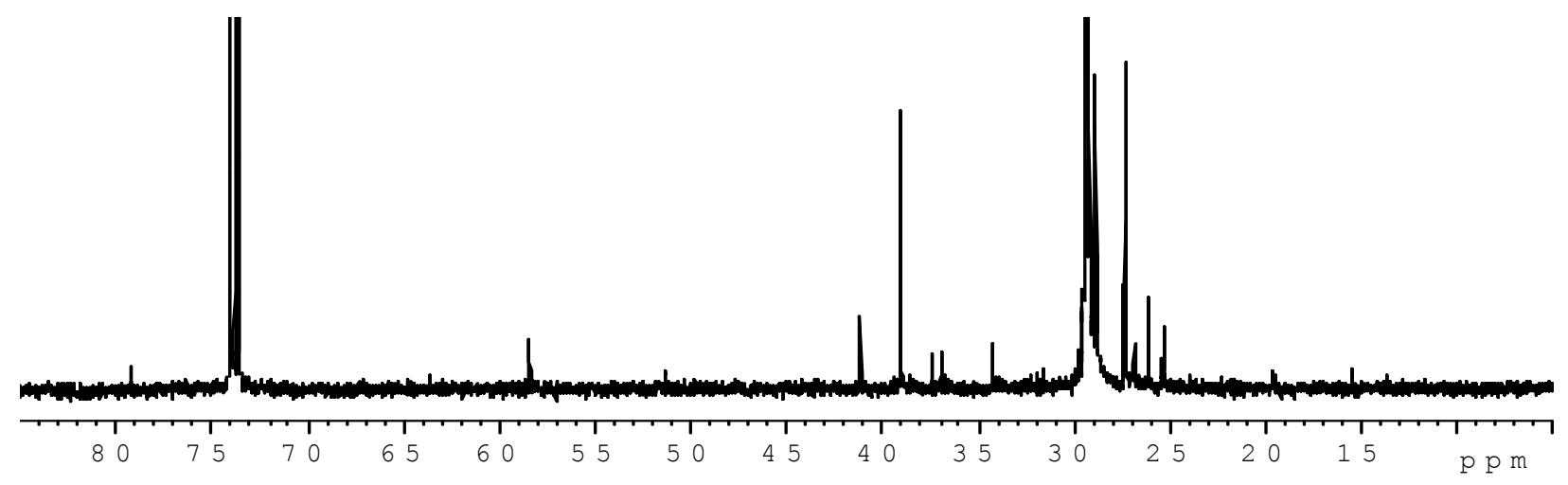

Figure S-6d. ${ }^{13} \mathrm{C} \mathrm{NMR}\left(\mathrm{CDCl}_{2} \mathrm{CDCl}_{2}, 100{ }^{\circ} \mathrm{C}\right)$ of ethylene $/ \mathrm{CH}_{2}=\mathrm{CHBr}$ copolymer: expansion of the $\delta 85-5$ region. The signal at $\delta 73.8$ is from the solvent. 


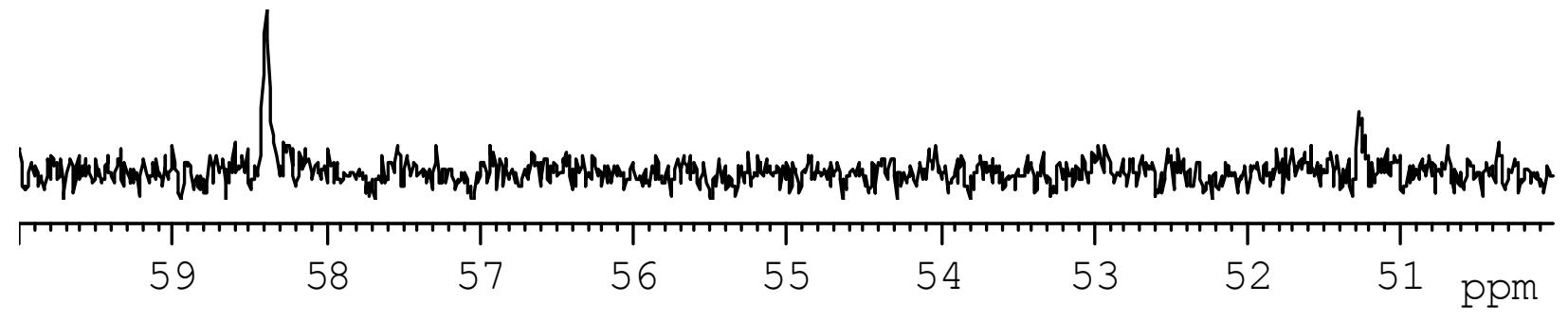

Figure S-6e. ${ }^{13} \mathrm{C}$ NMR $\left(\mathrm{CDCl}_{2} \mathrm{CDCl}_{2}, 100{ }^{\circ} \mathrm{C}\right)$ of ethylene $/ \mathrm{CH}_{2}=\mathrm{CHBr}$ copolymer: expansion of the $\delta 60-50$ region.

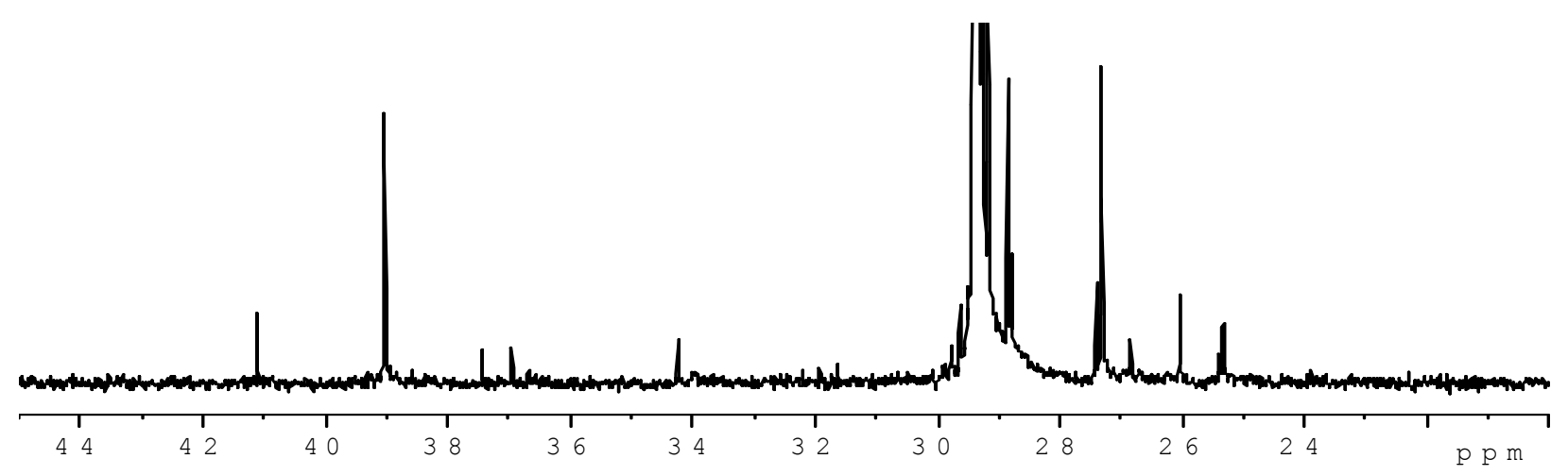

Figure S-6f. ${ }^{13} \mathrm{C} \mathrm{NMR}\left(\mathrm{CDCl}_{2} \mathrm{CDCl}_{2}, 100{ }^{\circ} \mathrm{C}\right)$ of ethylene $/ \mathrm{CH}_{2}=\mathrm{CHBr}$ copolymer: expansion of the $\delta$ 45-20 region.

Key NMR Data for Ethylene/ $\mathbf{C H}_{2}=\mathbf{C H O B u}$ Copolymer. ${ }^{\mathbf{1}} \mathbf{H} \mathbf{N M R}\left(\mathrm{CDCl}_{2} \mathrm{CDCl}_{2}, 100{ }^{\circ} \mathrm{C}\right.$, NMR400-1, SL310-1): $\delta 5.88\left(\mathrm{~m}, \mathrm{CH}_{2}=\mathrm{CHCH}_{2}-\right), 5.47\left(\mathrm{~m}, \mathrm{CH}_{3} \mathrm{CH}=\mathrm{CHCH}_{2}-\right), 5.05(\mathrm{~d}, J=17$, $\left.\mathrm{CH} \mathrm{H}^{\prime}=\mathrm{CH}-\right), 4.99\left(\mathrm{~d}, J=10, \mathrm{CH} H^{\prime}=\mathrm{CH}-\right), 3.45\left(\mathrm{t}, J=6, \mathrm{OCH}_{2} \mathrm{CH}_{2} \mathrm{CH}_{2} \mathrm{CH}_{3}\right), 3.41 \quad(\mathrm{~m}$, $\mathrm{CH}_{3} \mathrm{CH}(\mathrm{OBu}) \mathrm{CH}_{2-}$ ), 3.24 (quintet, $\left.J=6,-\mathrm{CH}_{2} \mathrm{CH}(\mathrm{OBu}) \mathrm{CH}_{2^{-}}\right), 2.11\left(\mathrm{~m}, \mathrm{CH}_{2}=\mathrm{CHCH}_{2^{-}}\right), 1.58(\mathrm{~m}$, $\left.J=8, \mathrm{OCH}_{2} \mathrm{CH}_{2} \mathrm{CH}_{2} \mathrm{CH}_{3}\right), 1.44\left(\mathrm{~m}, J=7, \mathrm{OCH}_{2} \mathrm{CH}_{2} \mathrm{CH}_{2} \mathrm{CH}_{3}\right), 1.16(\mathrm{~d}, J=6$, $\left.\mathrm{CH}_{3} \mathrm{CH}(\mathrm{OBu}) \mathrm{CH}_{2}-\right), 0.98\left(\mathrm{t}, J=7, \mathrm{OCH}_{2} \mathrm{CH}_{2} \mathrm{CH}_{2} \mathrm{CH}_{3}\right) \cdot{ }^{13} \mathbf{C}\left\{{ }^{1} \mathbf{H}\right\}$ NMR $\left(\mathrm{CDCl}_{2} \mathrm{CDCl}_{2}, 100{ }^{\circ} \mathrm{C}\right.$, NMR400-1, SL310-2): $\quad \delta \quad 79.3 \quad\left(-\mathrm{CH}_{2} \mathrm{CH}(\mathrm{OBu}) \mathrm{CH}_{2^{-}}\right), \quad 75.0 \quad\left(\mathrm{CH}_{3} \mathrm{CH}(\mathrm{OBu}) \mathrm{CH}_{2^{-}}\right), \quad 68.2$ $\left.\left(\mathrm{OCH}_{2} \mathrm{CH}_{2} \mathrm{CH}_{2} \mathrm{CH}_{3}\right), \quad 36.7 \quad\left(\mathrm{CH}_{3} \mathrm{CH}(\mathrm{OBu}) \mathrm{CH}_{2}-\right), \quad 34.1 \quad\left(-\mathrm{CH}_{2} \mathrm{CH}(\mathrm{OBu}) \mathrm{CH}_{2}\right)^{-}\right), \quad 32.3$ $\left(\mathrm{OCH}_{2} \mathrm{CH}_{2} \mathrm{CH}_{2} \mathrm{CH}_{3}\right), \quad 29.6 \quad\left(-\mathrm{CH}_{2} \mathrm{CH}_{2} \mathrm{CH}_{2} \mathrm{CH}(\mathrm{OBu}) \mathrm{CH}_{2} \mathrm{CH}_{2} \mathrm{CH}_{2}-\right), \quad 29.4 \quad\left(-\mathrm{CH}_{2}-\right), \quad 25.3$ 
$\left.\mathrm{CH}_{2} \mathrm{CH}_{2} \mathrm{CH}(\mathrm{OBu}) \mathrm{CH}_{2} \mathrm{CH}_{2}-\right), \quad 19.6\left(\mathrm{CH}_{3} \mathrm{CH}(\mathrm{OBu}) \mathrm{CH}_{2}-\right), \quad 19.2 \quad\left(\mathrm{OCH}_{2} \mathrm{CH}_{2} \mathrm{CH}_{2} \mathrm{CH}_{3}\right), \quad 13.6$ $\left(\mathrm{OCH}_{2} \mathrm{CH}_{2} \mathrm{CH}_{2} \mathrm{CH}_{3}\right)$; assignments confirmed by DEPT.

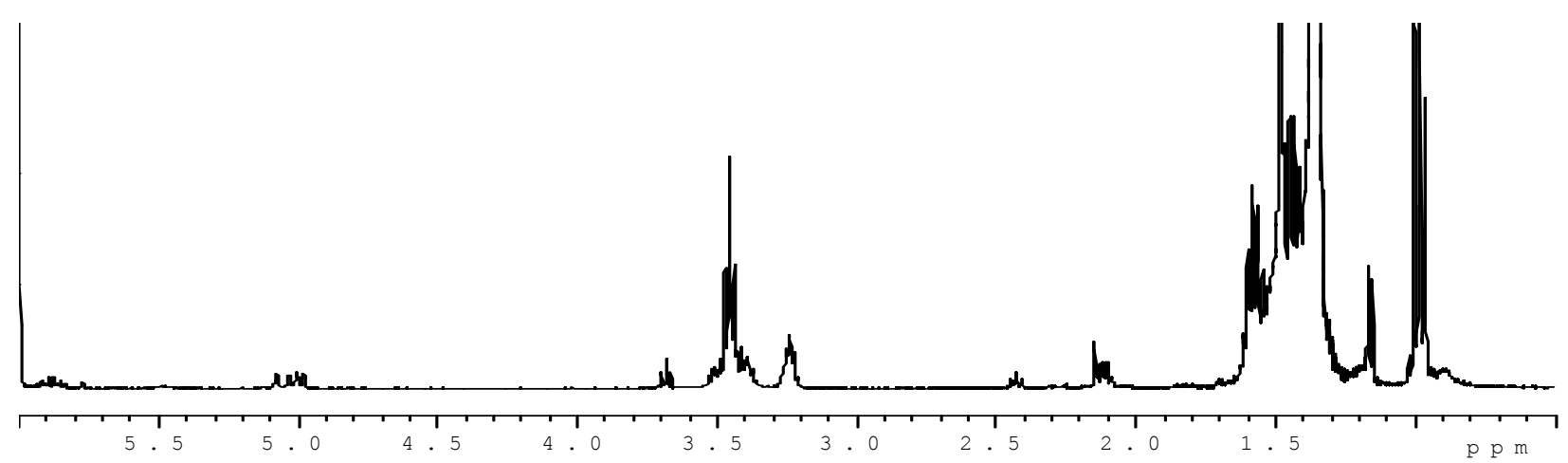

Figure S-7a. ${ }^{1} \mathrm{H}$ NMR $\left(\mathrm{CDCl}_{2} \mathrm{CDCl}_{2}, 100{ }^{\circ} \mathrm{C}\right)$ of ethylene $/ \mathrm{CH}_{2}=\mathrm{CHO}^{\mathrm{n}} \mathrm{Bu}$ copolymer: vertical expansion. The signal at $\delta 6.00$ is from the solvent.

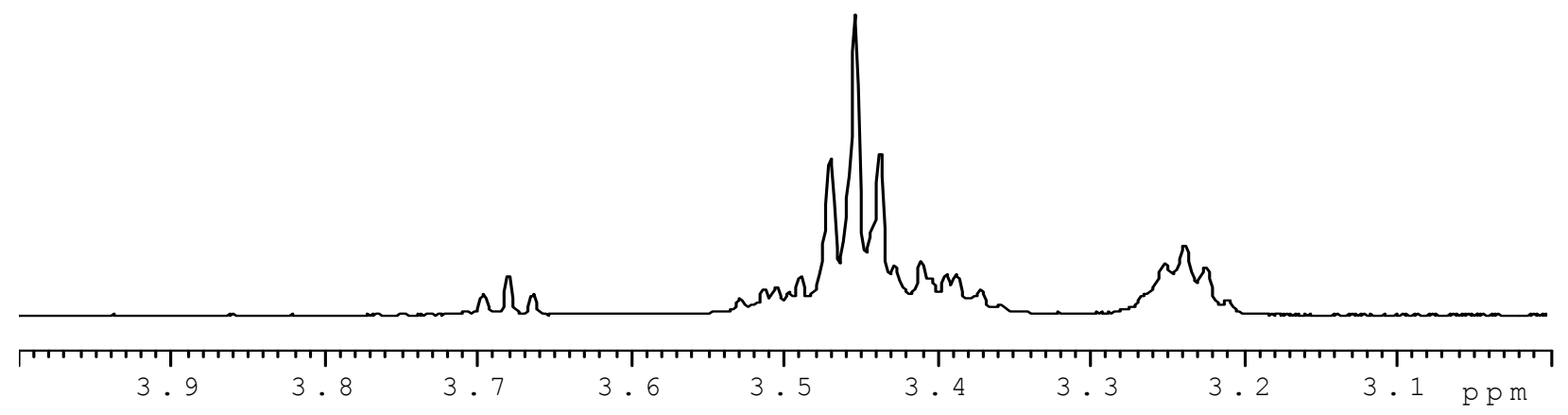

Figure S-7b. ${ }^{1} \mathrm{H}$ NMR $\left(\mathrm{CDCl}_{2} \mathrm{CDCl}_{2}, 100{ }^{\circ} \mathrm{C}\right)$ of ethylene $/ \mathrm{CH}_{2}=\mathrm{CHO}^{\mathrm{n}} \mathrm{Bu}$ copolymer: expansion of the $\delta$ 4.0-3.0 region. 


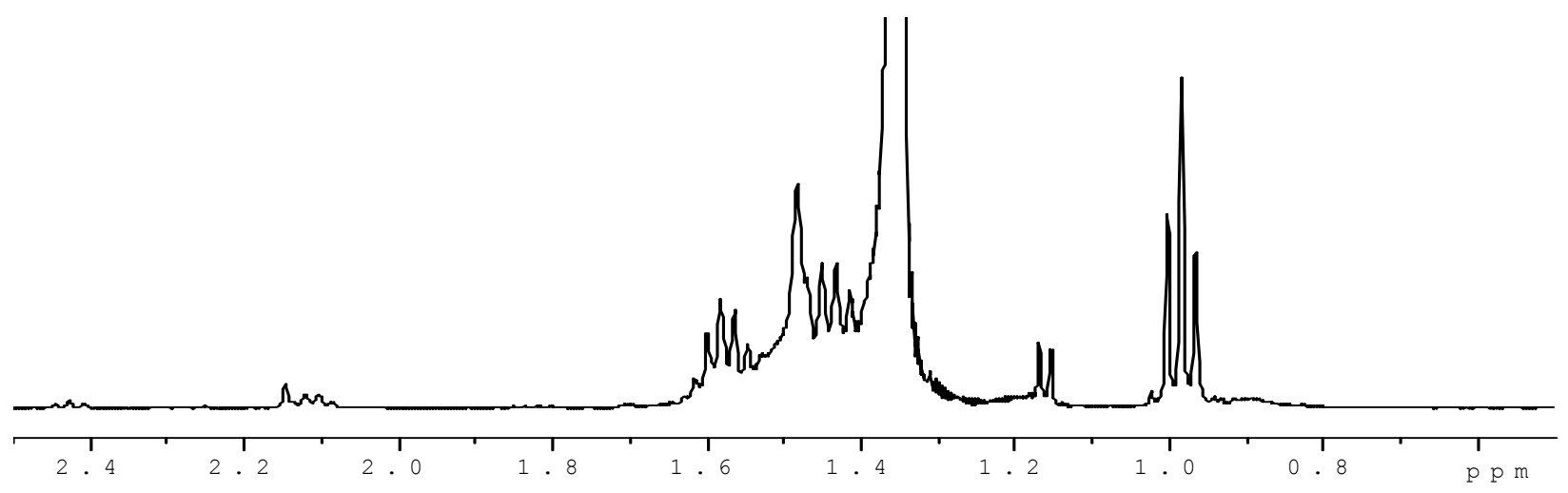

Figure S-7c. ${ }^{1} \mathrm{H} \mathrm{NMR}\left(\mathrm{CDCl}_{2} \mathrm{CDCl}_{2}, 100{ }^{\circ} \mathrm{C}\right)$ of ethylene $/ \mathrm{CH}_{2}=\mathrm{CHO}^{\mathrm{n}} \mathrm{Bu}$ copolymer: expansion of the $\delta$ 2.5-0.5 region.

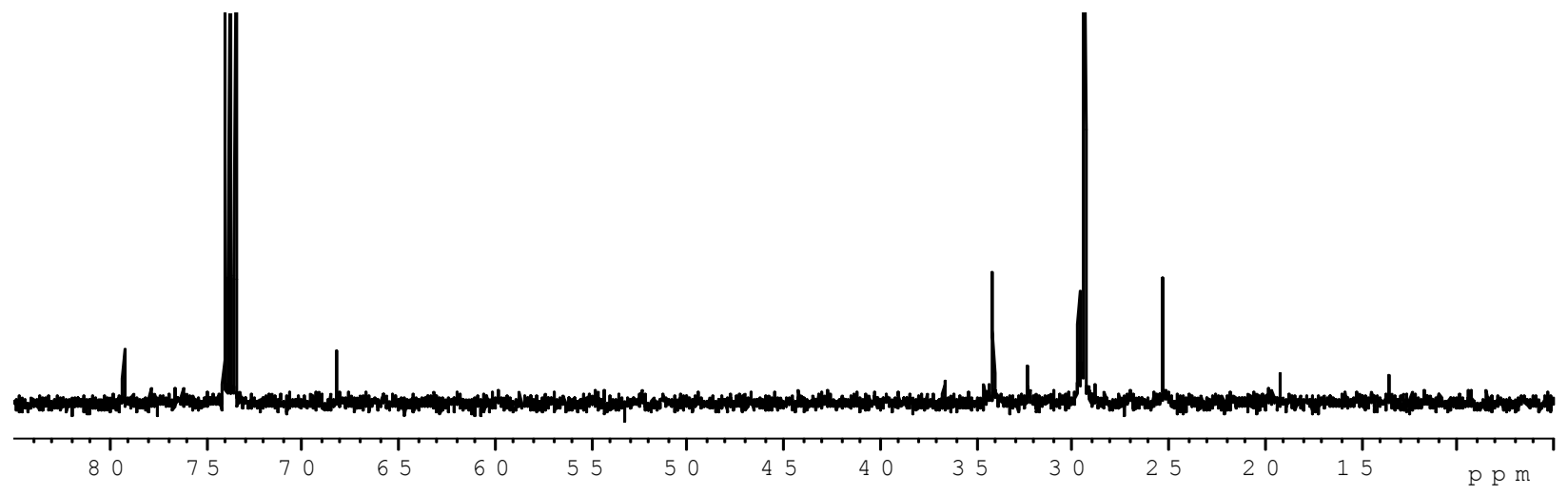

Figure S-7d. ${ }^{13} \mathrm{C} \mathrm{NMR}\left(\mathrm{CDCl}_{2} \mathrm{CDCl}_{2}, 100{ }^{\circ} \mathrm{C}\right)$ of ethylene $/ \mathrm{CH}_{2}=\mathrm{CHO}^{\mathrm{n}} \mathrm{Bu}$ copolymer: expansion of the $\delta 85-5$ region. The signal at $\delta 73.8$ is from the solvent. 


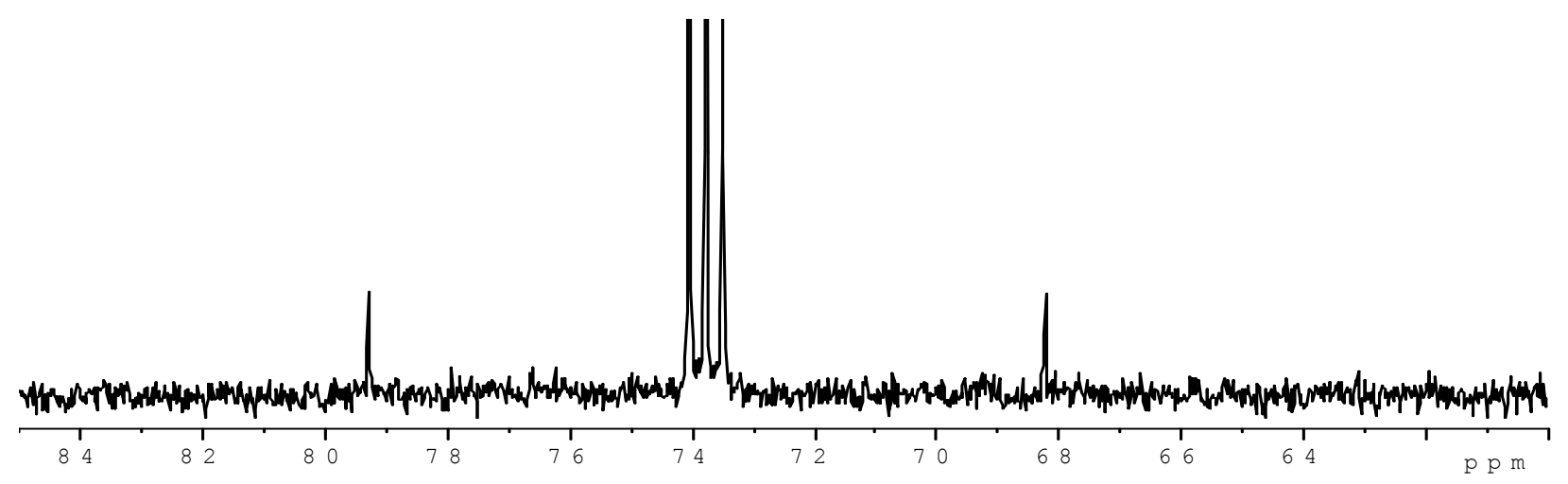

Figure S-7e. ${ }^{13} \mathrm{C} \mathrm{NMR}\left(\mathrm{CDCl}_{2} \mathrm{CDCl}_{2}, 100{ }^{\circ} \mathrm{C}\right)$ of ethylene $/ \mathrm{CH}_{2}=\mathrm{CHO}^{\mathrm{n}} \mathrm{Bu}$ copolymer: expansion of the $\delta 85-60$ region. The signal at $\delta 73.8$ is from the solvent.

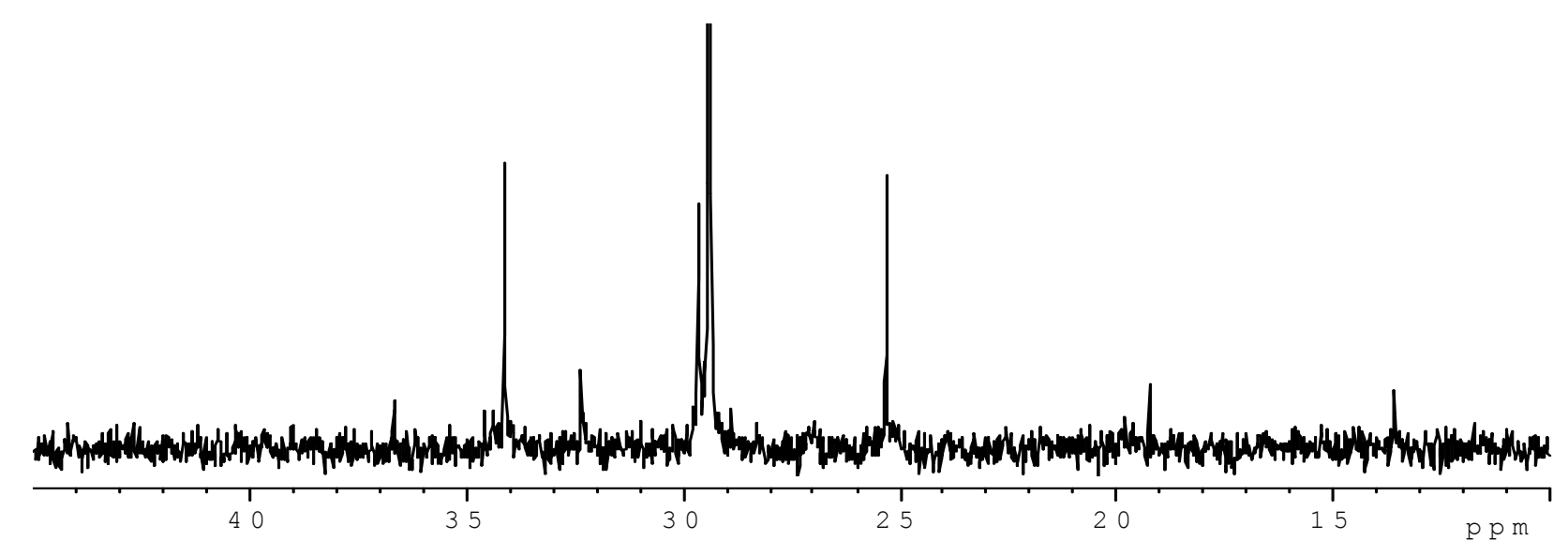

Figure S-7f. ${ }^{13} \mathrm{C}$ NMR $\left(\mathrm{CDCl}_{2} \mathrm{CDCl}_{2}, 100{ }^{\circ} \mathrm{C}\right)$ of ethylene $/ \mathrm{CH}_{2}=\mathrm{CHO}^{\mathrm{n}} \mathrm{Bu}$ copolymer: expansion of the $\delta 45-10$ region.

NMR Scale Batch Copolymerization of Ethylene with $\mathrm{CH}_{2}=\mathrm{CHOR}\left(\mathrm{R}=\mathrm{Et},{ }^{\mathrm{n}} \mathrm{Bu},{ }^{\mathrm{t}} \mathrm{Bu}, \mathrm{Ph}\right.$, $\mathbf{S i P h}_{3}$ ). NMR-scale batch copolymerization of ethylene and several vinyl ethers were conducted as control experiments for comparison to attempted (PO-OMe)PdMe(py)-catalyzed-vinyl ether homopolymerization, and for exploratory purposes. A general procedure for NMR scale copolymerizations is as follows (entry 2). An NMR tube was charged with (PO-OMe)PdMe(py) (3.0 mg, $0.0050 \mathrm{mmol})$ and toluene $(0.2 \mathrm{~mL}), \mathrm{CH}_{2}=\mathrm{CHO}^{\mathrm{n}} \mathrm{Bu}(7.5 \mathrm{mmol})$ and ethylene $(1.5 \mathrm{mmol})$ 
were added by vacuum-transfer at $-196^{\circ} \mathrm{C}$. The tube was warmed to $20^{\circ} \mathrm{C}$, shaken vigorously, and put into an $80{ }^{\circ} \mathrm{C}$ oil bath. After $19 \mathrm{~h}$, the reaction mixture was cooled to $20{ }^{\circ} \mathrm{C}$ and became cloudy. The volatiles were removed under vacuum and the nonvolatile residue was washed with $\mathrm{CH}_{2} \mathrm{Cl}_{2}$ /acetone, collected by filtration and dried under vacuum. Additional ethylene $/ \mathrm{CH}_{2}=\mathrm{CHOR}$ copolymerizations were carried out using this procedure. Representative results are listed in Table S-2.

Table $\boldsymbol{S}$-2. NMR scale copolymerization of ethylene and $\mathrm{CH}_{2}=\mathrm{CHOR}$ by (PO-OMe)PdMe(py)

\begin{tabular}{l|llllll}
\hline Entry & 1 & 2 & 3 & 4 & 5 & 6 \\
\hline Exp \# & $318(2)^{\mathrm{a}}$ & 310 & 309 & 315 & 313 & $304(2)$ \\
[Pd] $(\mu \mathrm{mol})$ & 5 & 5 & 5 & 5 & 5 & 5 \\
Ethylene (mmol) & 3.0 & 1.5 & 1.5 & 0 & 0.5 & 0.50 \\
$\mathrm{CH}_{2}=$ CHOR & $\mathrm{Et}$ & ${ }^{n} \mathrm{Bu}$ & ${ }^{\mathrm{t}} \mathrm{Bu}$ & ${ }^{\mathrm{t}} \mathrm{Bu}$ & $\mathrm{Ph}$ & $\mathrm{SiPh}_{3}$ \\
$\mathrm{CH}_{2}=$ CHOR (mmol) & 7.5 & 7.5 & 7.5 & 7.5 & 2.5 & 1.0 \\
Toluene (mL) & 0.2 & 0.2 & 0.2 & 0.2 & 0.7 & 0.4 \\
Comon. Incorp. (mol\%) & 5.4 & 8.1 & 2.5 & - & 1.5 & 0 \\
\hline
\end{tabular}

${ }^{\mathrm{a}} \mathrm{CH}_{2} \mathrm{Cl}_{2}$ was used as solvent.

Key NMR Data for Ethylene/ $\mathrm{CH}_{2}=\mathbf{C H O P h}$ Copolymer. ${ }^{\mathbf{1}} \mathbf{H} \mathbf{N M R}\left(\mathrm{CDCl}_{2} \mathrm{CDCl}_{2}, 100{ }^{\circ} \mathrm{C}\right.$, NMR400-1, SL313-1): $\delta 7.31\left(\mathrm{~m}, \mathrm{OC}_{6} \mathrm{H}_{5}\right), 7.05\left(\mathrm{~d}, \mathrm{OC}_{6} \mathrm{H}_{5}\right), 6.87\left(\mathrm{t}, \mathrm{OC}_{6} \mathrm{H}_{5}\right), 4.38$ (sextet, $J=6$, $\left.\mathrm{CH}_{3} \mathrm{CH}(\mathrm{OPh}) \mathrm{CH}_{2}-\right), 4.25$ (quintet, $\left.J=6,-\mathrm{CH}_{2} \mathrm{CH}(\mathrm{OPh}) \mathrm{CH}_{2^{-}}\right), 1.71\left(\mathrm{~d}, J=5, \mathrm{CH}_{3} \mathrm{CH}(\mathrm{OPh}) \mathrm{CH}_{2^{-}}\right.$ ) .

Control Experiment: Attempted Homopolymerization of $\mathrm{CH}_{2}=\mathrm{CHO}^{t} \mathrm{Bu}$ by (POOMe)PdMe(py). (Table S-2, entry 4, NB3-P49). An NMR tube was charged with (POOMe)PdMe(py) $(3.1 \mathrm{mg}, 0.0050 \mathrm{mmol})$ and toluene $(0.2 \mathrm{~mL})$, and $\mathrm{CH}_{2}=\mathrm{CHO}^{\mathrm{t}} \mathrm{Bu}(7.5 \mathrm{mmol})$ was added by vacuum-transfer at $-196{ }^{\circ} \mathrm{C}$. The tube was warmed to $20{ }^{\circ} \mathrm{C}$, shaken vigorously, and heated to $80{ }^{\circ} \mathrm{C}$ for $19 \mathrm{~h}$. The volatiles were removed under vacuum. ${ }^{1} \mathrm{H}$ NMR $\left(\mathrm{CDCl}_{3}\right)$ showed that no $-\left[\mathrm{CH}_{2} \mathrm{CH}\left(\mathrm{O}^{t} \mathrm{Bu}\right)\right]_{\mathrm{n}^{-}}$had formed. Under the same conditions (Table S-2, entry 3), ethylene $/ \mathrm{CH}_{2}=\mathrm{CHO}^{\mathrm{t}} \mathrm{Bu}$ copolymerization occurred.

Control Experiment: Attempted Copolymerization of Ethylene and $\mathrm{CH}_{2}=\mathrm{CHOEt}$ by 
AIBN. (NB3-P56). A Fisher-Porter bottle equipped with a stir bar was charged with AIBN (4.0 $\mathrm{mg}, 0.024 \mathrm{mmol}$ ), and $\mathrm{CH}_{2}=\mathrm{CHOEt}(1.43 \mathrm{~g}, 19.6 \mathrm{mmol})$ and toluene (total volume $25 \mathrm{~mL}$ ). The bottle was pressurized with ethylene (20 psi) and heated to $80{ }^{\circ} \mathrm{C}$ and stirred for $19 \mathrm{~h}$. The volatiles were removed, leaving a trace amount of $-\left[\mathrm{CH}_{2} \mathrm{CH}(\mathrm{OEt})\right]_{\mathrm{n}}$ - oligomer $(<2 \mathrm{mg})$. No ethylene incorporation was observed.

Control Experiment: Attempted NMR Scale Copolymerization of Ethylene and $\mathrm{CH}_{2}=$ CHOEt by $\left[\mathrm{Li}\left(\mathrm{OEt}_{2}\right)_{2.8}\right]\left[\mathbf{B}\left(\mathbf{C}_{6} \mathbf{F}_{5}\right)_{4}\right]$. (NB3-P56). An NMR tube was charged with $\left[\mathrm{Li}\left(\mathrm{OEt}_{2}\right)_{2}\right]\left[\mathrm{B}\left(\mathrm{C}_{6} \mathrm{~F}_{5}\right)_{4}\right](4.6 \mathrm{mg}, 0.0051 \mathrm{mmol})$ and $\mathrm{CH}_{2} \mathrm{Cl}_{2}(0.2 \mathrm{~mL})$, and $\mathrm{CH}_{2}=\mathrm{CHOEt}(7.5 \mathrm{mmol})$ and ethylene $(3.0 \mathrm{mmol})$ were added by vacuum-transfer at $-196^{\circ} \mathrm{C}$. The tube was warmed to 20 ${ }^{\circ} \mathrm{C}$, shaken vigorously, and heated to $80{ }^{\circ} \mathrm{C}$ for $19 \mathrm{~h}$. The volatiles were removed under vacuum to yield a clear viscous oil. ${ }^{1} \mathrm{H}$ NMR $\left(\mathrm{CDCl}_{3}\right)$ showed that the oil was $-\left[\mathrm{CH}_{2} \mathrm{CH}(\mathrm{OEt})\right]_{\mathrm{n}}{ }^{-}$. No ethylene incorporation was observed. Similar results were observed for $\left[\mathrm{H}\left(\mathrm{OEt}_{2}\right)_{2}\right]\left[\mathrm{B}\left(\mathrm{C}_{6} \mathrm{~F}_{5}\right)_{4}\right]$ and $\left[\mathrm{Ph}_{3} \mathrm{C}\right]\left[\mathrm{B}\left(\mathrm{C}_{6} \mathrm{~F}_{5}\right)_{4}\right]$.

1 De Graaf, W.; Boersma, J.; Smeets, Wilberth, J. J.; Spek, A. L.; Van Koten, G. Organometallics 1989, 8, 2907.

2 (a) $\mathrm{CH}_{2}=\mathrm{CHOSiPh}_{3}$ was reported previously by: Schaumann, E.; Tries, F. Synthesis 2002, 2, 191. (b) Data for $\mathrm{CH}_{2}=\mathrm{CHOSiPh}_{3}:{ }^{1} \mathrm{H}$ NMR $\left(\mathrm{CDCl}_{3}, \mathrm{NMR} 500-1, \mathrm{SL} 232-1\right) \delta 7.78(\mathrm{~d}, J=8$, $\left.6 \mathrm{H}, \mathrm{H}_{\text {ortho }}\right), 7.54$ (t, $\left.J=7,3 \mathrm{H}, \mathrm{H}_{\text {para }}\right), 7.49$ (t, $\left.J=7,6 \mathrm{H}, \mathrm{H}_{\text {meta }}\right), 6.65$ (dd, $\left.J=14,6 ; 1 \mathrm{H}, \mathrm{H}_{\text {int }}\right)$, $4.77\left(\mathrm{~d}, J=14,1 \mathrm{H}, \mathrm{H}_{\text {trans }}\right), 4.32$ (d, $\left.J=6,1 \mathrm{H}, \mathrm{H}_{\text {cis }}\right) .{ }^{13} \mathrm{C} \mathrm{NMR}\left(\mathrm{CDCl}_{3}, \mathrm{NMR} 500-1, \mathrm{SL} 232-2\right)$ $\delta 146.0(=C H O), 135.4,133.0\left(\mathrm{C}_{\mathrm{ipso}}\right), 130.4,128.0,95.8\left(\mathrm{CH}_{2}=\right)$. GC-MS: $m / z=302$.

${ }^{3}$ Data for $\mathrm{CH}_{2}=\mathrm{CHOPh}:{ }^{1} \mathrm{H}$ NMR $\left(\mathrm{CD}_{2} \mathrm{Cl}_{2}, \mathrm{NMR} 400-1,56-33\right): \delta 7.34$ (t, $\left.J=7,2 \mathrm{H}, \mathrm{H}_{\text {meta }}\right), 7.08$ (t, $\left.J=7,1 \mathrm{H}, \mathrm{H}_{\text {para }}\right), 7.00$ (d, $\left.J=8,2 \mathrm{H}, \mathrm{H}_{\text {ortho }}\right), 6.67$ (dd, $\left.J=14,6 ; 1 \mathrm{H}, \mathrm{H}_{\text {int }}\right), 4.75$ (d, $J=15$, $\left.1 \mathrm{H}, \mathrm{H}_{\text {trans }}\right), 4.43$ (d, $\left.J=6,1 \mathrm{H}, \mathrm{H}_{\text {cis }}\right)$. GC-MS: $m / z=120$.

${ }^{4}$ Okimoto, Y.; Sakaguchi, S.; Ishii, Y. J. Am. Chem. Soc. 2002, 124, 1590.

5 (a) Williamson, G. R.; Cervenka, A. Eur. Polym. J. 1972, 8, 1009. (b) Grinshpun, V.; Rudin, A. Makrom. Chem., Rapid Commun. 1985, 6, 219.

${ }^{6}$ (a) Katritzky, A. R.; Lopez-Rodriguez, M. L.; Keay, J. G.; and King, R. W. J. Chem. Soc., Perkin Trans. 2 1985, 2, 165. (b) Baughman, T. W.; Aa, E.; Wagener, K. B. Macromolecules 2006, 39, 7015. (c) Montoro, R.; Wirth, T. Synthesis 2005, 9, 1473. 
${ }^{7}$ Allen, N. T.; Goodall, B. L.; McIntosh, L. H. EP 1760086 A2.

${ }^{8}$ When $\mathrm{pH}<0$ was used during extraction, over-protonation of [PO-OMe]H to form [PO$\mathrm{OMe}] \mathrm{H}_{2}{ }^{+}$was observed by ${ }^{1} \mathrm{H}$ NMR.

${ }^{9}$ Vela, J.; Lief, G. R.; Shen, Z.; Jordan, R. F. manuscript in preparation.

10 The exchange could not be frozen out since at lower temperatures $\left(-80 \sim-20{ }^{\circ} \mathrm{C}\right)$, insoluble species formed.

${ }^{11}$ The chemical shifts for coordinated $\mathrm{CH}_{2}=\mathrm{CHOEt}$ were estimated by the equation: $\delta_{\text {obs }}=$ $\delta_{\text {free }} \chi_{\text {free }}+\delta_{\text {free }} \chi_{\text {coord }}$, where $\chi_{\text {free }}$ and $\chi_{\text {coord }}$ are the mole fractions of free and coordinated $\mathrm{CH}_{2}=$ CHOEt. ${ }^{1} \mathrm{H}$ NMR data $\left(\mathrm{CD}_{2} \mathrm{Cl}_{2}\right)$ for free $\mathrm{CH}_{2}=\mathrm{CHOEt}: \delta 6.46\left(\mathrm{dd}, 1 \mathrm{H}, \mathrm{H}_{\text {int }}\right), 4.15(\mathrm{~d}, 1 \mathrm{H}$, $\left.\mathrm{H}_{\text {trans }}\right), 3.96\left(\mathrm{~d}, 1 \mathrm{H}, \mathrm{H}_{\text {cis }}\right), 3.73\left(\mathrm{q}, 2 \mathrm{H}, \mathrm{OCH}_{2} \mathrm{CH}_{3}\right), 1.16\left(\mathrm{t}, 1 \mathrm{H}, \mathrm{OCH}_{2} \mathrm{CH}_{3}\right)$.

${ }^{12}$ Key NMR data for $\left[(\alpha\right.$-diimine $\left.) \mathrm{Pd}\left\{\mathrm{CH}_{2} \mathrm{CH}(\mathrm{OEt}) \mathrm{Me}\right\}\right]\left[\mathrm{B}\left(\mathrm{C}_{6} \mathrm{~F}_{5}\right){ }_{4}\right]:{ }^{1} \mathrm{H} \mathrm{NMR}\left(\mathrm{CD}_{2} \mathrm{Cl}_{2}\right): \delta 4.86$ (sextet, $\left.J=7, \mathrm{PdCH}_{2} \mathrm{CH}(\mathrm{OEt}) \mathrm{Me}\right), 0.37$ (t, $J=7$, $\left.\mathrm{PdCH} \mathrm{H}^{\prime} \mathrm{CH}(\mathrm{OEt}) \mathrm{Me}\right)$.

${ }^{13}$ In one case, the copolymer was contaminated with ca. $5 \mathrm{~mol} \%$ of the $-\left[\mathrm{CH}_{2} \mathrm{CH}(\mathrm{OEt})\right]_{\mathrm{n}^{-}}$ homopolymer, which was probably generated by adventitious $\mathrm{H}^{+}$species. The mixture was split into two portions, and the $-\left[\mathrm{CH}_{2} \mathrm{CH}(\mathrm{OEt})\right]_{\mathrm{n}}$ - homopolymer was completely removed by either Soxhlet extraction using $\mathrm{CHCl}_{3}$ (portion 1) or by stirring in $\mathrm{CHCl}_{3}$ at $50-60{ }^{\circ} \mathrm{C}$ overnight followed by filtration (portion 2).

${ }^{14}$ It was not possible to obtain good HMQC data due to the thermal conversion of the ethylene/ $\mathrm{CH}_{2}=\mathrm{CHO}^{\mathrm{t}} \mathrm{Bu}$ copolymer to the ethylene $/ \mathrm{CH}_{2}=\mathrm{CHOH}$ copolymer.

${ }^{15}$ The $-\left[\mathrm{CH}_{2} \mathrm{CH}\left(\mathrm{O}^{t} \mathrm{Bu}\right)\right]_{\mathrm{n}}-$ homopolymer was prepared by the reaction of $(\alpha$-diimine $) \mathrm{PdMe}^{+}(\alpha-$ diimine $\left.=\left(2,6-{ }_{-}^{\mathrm{i}} \mathrm{Pr}_{2}-\mathrm{C}_{6} \mathrm{H}_{3}\right) \mathrm{N}=\mathrm{CMeCMe}=\mathrm{N}\left(2,6-{ }_{-}^{\mathrm{i}} \mathrm{Pr}_{2}-\mathrm{C}_{6} \mathrm{H}_{3}\right)\right)$ with excess $\mathrm{CH}_{2}=\mathrm{CHO}^{\mathrm{t}} \mathrm{Bu}$ in $\mathrm{CH}_{2} \mathrm{Cl}_{2}$ at $20^{\circ} \mathrm{C}$. See Luo, S.; Jordan, R. F. J. Am. Chem. Soc. 2006, 128, 12072.

${ }^{16}$ Literature on NMR of the $-\left[\mathrm{CH}_{2} \mathrm{CH}\left(\mathrm{O}^{t} \mathrm{Bu}\right)\right]_{\mathrm{n}}$ - homopolymer: (a) Zhou, Y.; Faust, R.; Chen, S.; Gido, S. P. Macromolecules 2004, 37, 6716. (b) Palos, I.; Pliego, G. C.; Knjazhanki, S. Y.; Regalado, E. J.; Casa, E. G.; Ibarra, V. H. Polym. Degrad. Stab. 2005, 90, 264.

${ }^{17}{ }^{1} \mathrm{H} \mathrm{NMR}\left(\mathrm{CDCl}_{2} \mathrm{CDCl}_{2}, 100{ }^{\circ} \mathrm{C}\right)$ for isobutene: $\delta 4.74\left(\mathrm{~m}, J=1, \mathrm{CH}_{2}=\mathrm{CMe}_{2}\right), 1.80(\mathrm{q}, J=1$, $\left.\mathrm{CH}_{2}=\mathrm{CMe}_{2}\right)$.

${ }^{18}$ The correlation of $\delta 3.63$ and $\delta 1.46$ was determined by homonuclear decoupling experiments.

${ }^{19}{ }^{1} \mathrm{H}$ NMR $\left(\mathrm{CDCl}_{2} \mathrm{CDCl}_{2}, 100{ }^{\circ} \mathrm{C}\right)$ for ${ }^{\mathrm{t}} \mathrm{BuCl}: \delta 1.69$ (s). 
${ }^{20}{ }^{1} \mathrm{H} \mathrm{NMR}\left(\mathrm{CDCl}_{2} \mathrm{CDCl}_{2}, 100{ }^{\circ} \mathrm{C}\right)$ for $\mathrm{CH}_{3} \mathrm{CH}(\mathrm{Cl}) \mathrm{CH}_{2}$ - (identified by comparison to data for $\mathbf{S}-$ 7, Chart S-1): $\delta 4.09$ (sextet, $\left.J=6, \mathrm{CH}_{3} \mathrm{CH}(\mathrm{Cl}) \mathrm{CH}_{2^{-}}\right), 1.80\left(\mathrm{~m}, \mathrm{CH}_{3} \mathrm{CH}(\mathrm{Cl}) \mathrm{CH}_{2^{-}}\right), 1.57$ (d, $J=$ 7, $\left.\mathrm{CH}_{3} \mathrm{CH}(\mathrm{Cl}) \mathrm{CH}_{2}-\right)$.

${ }^{21}$ After heating at $120{ }^{\circ} \mathrm{C}$ for $36 \mathrm{~h}, 40 \%$ of the vinyl groups $\left(-\mathrm{CH}_{2} \mathrm{CH}=\mathrm{CH}_{2}\right)$ were converted to 2olefins $\left(-\mathrm{CH}_{2} \mathrm{CH}=\mathrm{CHMe}\right)$.

${ }^{22}$ Chen, C. L.; Chen, Y. C.; Liu, Y. H.; Peng, S. M.; Liu, S. T. Organometallics 2002, 21, 5382. 